\title{
Efficient Failure Probability Calculations and Modeling Interface Debonding in TRISO Particles with Bison
}

\section{September 2021}

Technical Report

Wen Jiang ${ }^{1}$, Gyanender Singh ${ }^{1}$, Jason D. Hales ${ }^{1}$, Aysenur Toptan ${ }^{1}$, Benjamin W. Spencer ${ }^{1}$, and Stephen R. Novascone ${ }^{1}$

${ }^{1}$ Idaho National Laboratory

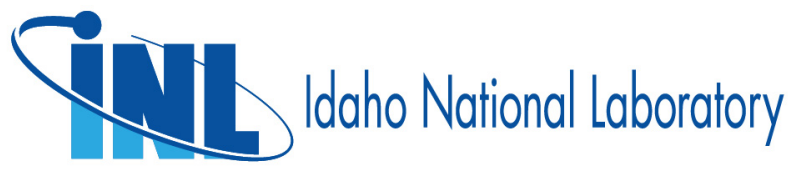


DISCLAIMER

This information was prepared as an account of work sponsored by an agency of the U.S. Government. Neither the U.S. Government nor any agency thereof, nor any of their employees, makes any warranty, expressed or implied, or assumes any legal liability or responsibility for the accuracy, completeness, or usefulness, of any information, apparatus, product, or process disclosed, or represents that its use would not infringe privately owned rights. References herein to any specific commercial product, process, or service by trade name, trade mark, manufacturer, or otherwise, does not necessarily constitute or imply its endorsement, recommendation, or favoring by the U.S. Government or any agency thereof. The views and opinions of authors expressed herein do not necessarily state or reflect those of the U.S. Government or any agency thereof. 


\title{
Efficient Failure Probability Calculations and Modeling Interface Debonding in TRISO Particles with Bison
}

\author{
Technical Report
}

Wen Jiang ${ }^{1}$, Gyanender Singh ${ }^{1}$, Jason D. Hales ${ }^{1}$, Aysenur Toptan ${ }^{1}$, Benjamin W. Spencer ${ }^{1}$, and Stephen R. Novascone ${ }^{1}$

${ }^{1}$ Idaho National Laboratory

30 September 2021

Idaho National Laboratory

Computational Mechanics and Materials Department

Idaho Falls, Idaho 83415

http://www.inl.gov

Prepared for the

U.S. Department of Energy

Office of Nuclear Energy

Under U.S. Department of Energy-Idaho Operations Office

Contract DE-AC07-05ID14517 


\begin{abstract}
This document constitutes completion of the NEAMS milestone, which is titled: Demonstrate efficiency improvements in TRISO failure probability calculations and the effect of debonding on fission product transport in TRISO fuel particles in Bison. In this report we present the development of : (1) the anisotropic elasticity model of pyrolytic carbon and extended material models using the local coordinate system for aspherical particle geometry, (2) a capability of modeling interface debonding in TRISO particles , (3) an efficient high-fidelity approach to calculate the failure probability of TRISO particles. Benchmark problems and AGR-2 tests are used to verify the models and demonstrate these new capabilities.
\end{abstract}




\section{ACKNOWLEDGMENT}

The submitted manuscript was authored by a contractor of the U.S. Government under contract DE-AC0705ID14517. Accordingly, the U.S. Government retains a non-exclusive, royalty-free license to publish or reproduce the published form of this contribution, or allow others to do so, for U.S. Government purposes.

This research made use of the resources of the High Performance Computing Center at INL, which is supported by the Office of Nuclear Energy of the U.S. Department of Energy and the Nuclear Science User Facilities under contract DE-AC07-05ID14517.

\section{DECLARATION OF COMPETING INTEREST}

The authors declare that they have no known competing financial interests or personal relationships that could have appeared to influence the work reported in this technical report.

ORCID

\begin{tabular}{|c|c|}
\hline Wen Jiang & orcid.org/0000-0001-6978-9159 \\
\hline Gyanender Singh & orcid.org/0000-0003-1828-4438 \\
\hline Jason D. Hales & orcid.org/0000-0003-0836-0476 \\
\hline Aysenur Toptan & orcid.org/0000-0003-4250-6336 \\
\hline & orcid.org/0000-0002-8774-4445 \\
\hline Novasce & orcid.org/0000-0002-3938-4326 \\
\hline
\end{tabular}




\section{CONTENTS}

Abstract

List of Figures

List of Tables

Acronyms

I Introduction 2

II TRISO Material Model Improvement 2



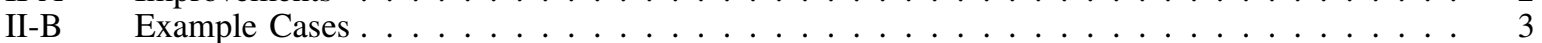

II-B1 Incorporating anisotropy in the elasticity model of the PyC layer . . . . . . . . . 3

II-B2 Overall improvements in the calculations . . . . . . . . . . . . . . . 4

II-C Thermal Anisotropy . . . . . . . . . . . . . . . . . . . . . . . . 9

III Modeling Debonding in TRISO Fuel Particles 10

III-A Mesh Modifier for Creating Cohesive Zone Interfaces . . . . . . . . . . . . . . . . . . 10

III-B Bilinear Mixed-Mode Traction Separation Law . . . . . . . . . . . . . . . . . . . . 10

III-B1 Softening onset prediction . . . . . . . . . . . . . . . . . . 11

III-B2 Delamination propagation prediction . . . . . . . . . . . . . . . 11

III-B3 Constitutive equation for mixed-mode loading . . . . . . . . . . . . . . . . . . 12

III-B4 Viscous regularization . . . . . . . . . . . . . . . . . . . . . . 12

III-C Thermal Heat Transfer between Debonded Layers . . . . . . . . . . . . . . . . . . . . . 12

III-D TRISO Layer Debonding Examples . . . . . . . . . . . . . . . . . . . . . . 13

III-D1 Debonding of the IPyC from the $\mathrm{SiC} \ldots \ldots \ldots \ldots \ldots$

III-D2 Debonding of the buffer from the IPyC . . . . . . . . . . . . . . . . . 14

III-D3 Debonding of the buffer from the IPyC and IPyC cracking . . . . . . . . . . . . . 20

III-D4 Debonding of the buffer, IPyC, and SiC layers . . . . . . . . . . . . . . . . . 21

III-E Incorporate Debonding Failure into Monte Carlo Scheme . . . . . . . . . . . . . . . . . . 22

III-E1 Stress correlation for IPyC cracking . . . . . . . . . . . . . . . . . . . . . 22

III-E2 Monte Carlo Scheme . . . . . . . . . . . . . . . . . . . . 23

IV Efficient High-fidelity TRISO Statistical Failure Analysis 28

IV-A Failure Modes . . . . . . . . . . . . . . . . . . . . . . . 28

IV-A1 Pressure vessel failure . . . . . . . . . . . . . . . . . . . . . 28

IV-A2 Irradiation-induced IPyC failure leading to SiC cracking . . . . . . . . . . . . . 28

IV-A3 Pressure vessel failure of an aspherical particle . . . . . . . . . . . . . . 28

IV-B Weibull Failure Theory . . . . . . . . . . . . . . . . . . . . . . . . . 28

IV-C Higher Order Stress Concentrations . . . . . . . . . . . . . . . . . . . . . . . . 30

IV-C1 Stress correlation for IPyC cracking . . . . . . . . . . . . . . . . . . . 31

IV-C2 Stress correlation for an aspherical particle . . . . . . . . . . . . . . . 31

IV-D Direct Integration Scheme . . . . . . . . . . . . . . . . . . . . 32

IV-D1 Integration of failure probability function . . . . . . . . . . . . . . . . 32

IV-D2 MOOSE Stochastic Tools module . . . . . . . . . . . . . . . . 33

IV-D3 Methodology ......................... 33

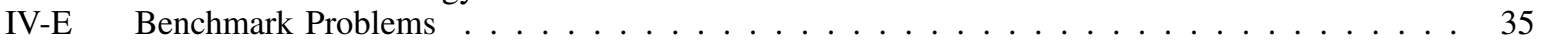

IV-F AGR-2 Irradiation As-run Prediction . . . . . . . . . . . . . . . . . . . 38

V Conclusion and Future Work 47

Bibliography 48 


\section{LIST OF FIGURES}

Concept of local coordinate axes in a general layered-type geometry and a TRISO particle. . . . . . . Variation of maximum radial stress with fluence in the IPyC, $\mathrm{SiC}$, and $\mathrm{OPyC}$ layers of a spherical TRISO particle and an aspherical TRISO particle.

Variation of maximum tangential stress with fluence in the IPyC, $\mathrm{SiC}$, and $\mathrm{OPyC}$ layers of a spherical TRISO particle and an aspherical TRISO particle. . . . . . . . . . . . . . Variation of failure probability with fluence in the IPyC and SiC layers of a spherical TRISO particle and an aspherical TRISO particle. . . . . . . . . . . . . . . . . . Variation of maximum radial and tangential stresses with fluence in the $\mathrm{IPyC}, \mathrm{SiC}$, and $\mathrm{OPyC}$ layers. Variation of failure probability with fluence in the IPyC layer. . . . . . . . . . . . . Three-block examples . . . . . . . . . . . . . . . . . . . . . . Temperature contour plots with varying fill gas conductivity from the two-block verification example. Temperature predictions across the center horizontal line from the two-block verification example. . . Random scale factor for normal strength. . . . . . . . . . . . . . . . Damage evolution during debonding of the $\mathrm{IPyC}$ from the $\mathrm{SiC} \ldots \ldots \ldots \ldots \ldots$ Radial stress in the IPyC, $\mathrm{SiC}$, and OPyC layers during debonding of the IPyC from the $\mathrm{SiC} \ldots \ldots$ Tangential stress in the $\mathrm{SiC}$ layer during debonding of the $\mathrm{IPyC}$ from the $\mathrm{SiC} \ldots \ldots \ldots$ Time history of stress in the $\mathrm{SiC}$ layer during debonding of the $\mathrm{IPyC}$ from the $\mathrm{SiC} \ldots \ldots \ldots \ldots$ Damage evolution during debonding of the IPyC from the $\mathrm{SiC}$ in an aspherical particle . . . . . . . Damage evolution during debonding of the buffer from the IPyC with a bond strength of $80 \mathrm{MPa}$. Radial stress during debonding of the buffer from the IPyC with a bond strength of $80 \mathrm{MPa} \ldots \ldots$ Time history of stress in the IPyC layer during debonding of the buffer from the IPyC . . . . . . . . Partial debonding of the buffer from the IPyC layer. . . . . . . . . . . . . . . . 20 Damage evolution during partial debonding of the buffer from the $\mathrm{IPyC} \ldots \ldots \ldots \ldots$ Tangential stress during partial debonding of the buffer from the IPyC $\ldots \ldots \ldots \ldots \ldots \ldots . \ldots 21$ Time history of stress in the IPyC layer during partial debonding of the buffer from the IPyC. . . . 21 Damage evolution during debonding of the buffer from the IPyC and IPyC cracking . . . . . . . 22 Tangential stress during debonding of the buffer from the IPyC and IPyC cracking . . . . . . . 22 Time history of stress during debonding of the buffer from the IPyC and IPyC cracking. . . . . . . 23 Damage evolution during debonding of the buffer from the IPyC and the IPyC from the SiC layer . . 24 Radial stress during debonding of the buffer from the IPyC and the IPyC from the SiC layer . . . . 25 Time history of stress during debonding of the buffer, IPyC, and $\mathrm{SiC}$ layers. . . . . . . . . . 26 Time histories of maximum tangential stress in the $\mathrm{SiC}$ layer for a 2-D model of a particle with debonding between the IPyC and $\mathrm{SiC}$ layers, as compared with a 1-D model of an intact particle. . . 26 MC scheme employed by Bison for calculating the failure probability of TRISO particles. . . . . . 27 2-D axisymmetric model of postulated cracking of the IPyC layer, and comparison between applying

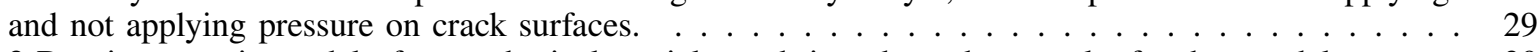
2-D axisymmetric model of an aspherical particle, and time-dependent results for that model. . . . . . Example of stress correlation functions for IPyC cracking. . . . . . . . . . . . . . Example of stress correlation functions for an aspherical particle. . . . . . . . . . . . . . . . 32 Integration values using MOOSE's sparse grid with different orders of the polynomials. . . . . . . . . 34 Quadrature point location. . . . . . . . . . . . . . . . . . . . . . 34 Calculate the failure probability of TRISO particles in Bison: direct integration scheme using 1-D simulation with correlation functions. . . . . . . . . . . . . . . . Calculate the failure probability of TRISO particles in Bison: direct integration scheme using 2-D

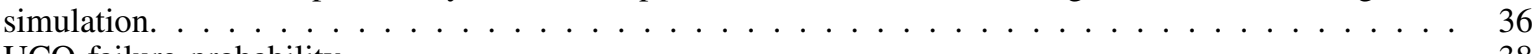

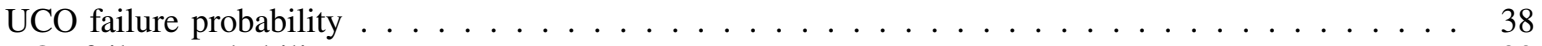


Linear regression results for UCO compacts: (a) temperature vs. probability of IPyC cracking, (b) fluence vs. probability of IPyC cracking, and (c) burnup vs. probability of IPyC cracking. . . . . . . 42 Linear regression results for UCO compacts: (a) temperature vs. probability of IPyC cracking, (b) fluence vs. probability of IPyC cracking, and (c) burnup vs. probability of overall $\mathrm{SiC}$ failure. . . . . 42 
44 Inner-wall tangential stress histories in the SiC layer for AGR-2 Capsule 5 compacts. . . . . . . . . . 44

45 Inner-wall tangential stress histories in the SiC layer for AGR-2 Capsule 3 compacts. . . . . . . . . . 45

46 Inner-wall tangential stress histories in the SiC layer for AGR-2 Capsule 2 compacts. . . . . . . . . . 46

\section{LIST OF TABLES}

I Simulation settings for the CZM properties. . . . . . . . . . . . . . . . . 13

II Number of quadrature points for tensor grid. . . . . . . . . . . . . . . . . . . . 33

III Irradiation conditions for benchmark problems. . . . . . . . . . . . . . . . . . 35

IV Fuel parameters in benchmark problems. . . . . . . . . . . . . . . . . $\ldots$

V Stress correlation factors and effective mean strength for benchmark problems. . . . . . . . . . . 37

VI Quadractic stress correlation functions for benchmark problems. . . . . . . . . . . . . . . . 37

VII IPyC cracking probability for benchmark problems. . . . . . . . . . . . . . . . . . . 37

VIII Failure probability for benchmark problems using Bison's direct integration scheme. . . . . . . . . 38

IX UCO and $\mathrm{UO}_{2}$ parameters used in modeling the AGR-2 irradiation. . . . . . . . . . . . . . . . 39

X Compacts used in modeling the AGR-2 irradiation. . . . . . . . . . . . . . . . . . . . 40

XI Failure probability for AGR-2 compacts. . . . . . . . . . . . . . . . . . 41 


\section{ACRONYMS}

$\begin{array}{ll}\text { AGR } & \text { Advanced Gas Reactor } \\ \text { CZM } & \text { cohesive zone modeling } \\ \text { EFPD } & \text { effective full power days } \\ \text { IIDC } & \text { irradiation-induced dimensional changes } \\ \text { INL } & \text { Idaho National Laboratory } \\ \text { IPyC } & \text { inner pyrolytic carbon (PyC) } \\ \text { MC } & \text { Monte Carlo } \\ \text { MOOSE } & \text { Multiphysics Object-Oriented Simulation Environment } \\ \text { NASA } & \text { National Aeronautics and Space Administration } \\ \text { OPyC } & \text { outer PyC } \\ \text { PDF } & \text { probability density function } \\ \text { PIA } & \text { principle of independent action } \\ \text { POD } & \text { proper orthogonal decomposition } \\ \text { PVF } & \text { pressure vessel failure } \\ \text { PyC } & \text { pyrolytic carbon } \\ \text { SiC } & \text { silicon carbide } \\ \text { TRISO } & \text { tri-structural isotropic } \\ \text { 1-D } & \text { one-dimensional } \\ \text { 2-D } & \text { two-dimensional }\end{array}$




\section{INTRODUCTION}

Over the last few years, the Bison fuel performance code [1] has undergone significant development to expand its tri-structural isotropic (TRISO) particle fuel performance modeling capabilities [2, 3, 4, 5, 6, 7, 8]. Such development included the implementation of material models from PARFUME [9] and the capability to perform statistical failure analysis on large sets of samples, utilizing a Monte Carlo (MC) scheme to execute fast-running, one-dimensional (1-D), spherically symmetric models. Some PARFUME material models are simplified to be isotropic and make 1-D assumptions that only work for perfectly spherical particles. Improvements are needed in these material models to enable accurate analysis of TRISO particles. Several potential failure mechanisms for TRISO fuel, including cracking of the inner PyC (IPyC) layer and pressure vessel failure of an aspherical particle, were considered in Bison failure analysis. Debonding is another important failure mechanism observed in recent Advanced Gas Reactor (AGR) experiments. For Bison, a capability must be developed for modeling debonding in TRISO and incorporating it into statistical failure analysis. One limitation of the $\mathrm{MC}$ scheme in predicting particle failure is that the number of necessary samples is very large to compute a relatively low failure probability, and the computational resources needed for the task are tremendous. Therefore, it is important that Bison be enhanced with a fast integration approach to greatly enhance the efficiency of failure probability calculations.

The recent development to improve TRISO failure analysis in Bison is summarized in this report, which begins with Section II describing improvements to material models to eliminate isotropic and 1-D spherical particle assumptions. Section III outlines the new capability to model interface debonding in TRISO particles by using Bison. Several debonding examples are considered for demonstration. The direct integration approach of calculating failure probability is described in Section IV. To demonstrate its improved efficiency, the new approach is used to compute the stress and failure probability of 48 AGR-2 compacts. Finally, a conclusion and discussion of future work are provided in Section V.

\section{TRISO MATERIAL MODEL IMPROVEMENT}

The original TRISO capability in Bison assumed isotropy for some of the material models; namely, elasticity and thermal models for PyC. Also, some other models (e.g., the models for irradiation-induced swelling and thermal expansion of PyC) assumed perfectly spherical geometry. These models were initially developed for PARFUME, based on 1-D assumptions. However, as reported in [9, 10], the PyC layer in a TRISO particle exhibits anisotropy, which can significantly alter the predicted mechanical and thermal behavior of a TRISO particle. Also, TRISO particles generally deviate from a perfect spherical geometry, and in many cases, the asphericity can be significant enough to invalidate the assumption of perfect spherical geometry. Thus, improvements were needed in these material models to enable accurate analysis of the TRISO particles.

\section{A. Improvements}

To incorporate anisotropy in a regular spherical or non-spherical TRISO model, the material properties along the radial and tangential directions must be defined. In finite element software, the material properties are defined along a particular direction, using a system of coordinate axes. A local coordinate axes system defines coordinate axes at every location in a finite element model. In Bison, the capability to define local coordinate axes at every location in a TRISO particle was developed. Using this local coordinate system, the material properties (e.g., the elastic modulus) and strains tensors could be defined and then transformed into the global coordinate system for finite element calculations. The details of this approach are described in the schematic shown in Figure 1.

Figure 1a shows a layer of material in which the local coordinate system is defined in such a way that one local coordinate axis is aligned along the layer, while another axis is aligned in the transverse direction to the layer. Using this coordinate system, anisotropic properties can be defined for this layer (e.g., an elastic modulus of $100 \mathrm{GPa}$ along the layer, and $40 \mathrm{GPa}$ in the direction perpendicular to the layer). Similarly, for the PyC layer in a TRISO particle, different properties can be defined along the radial and tangential directions, as shown in Figure $1 b$. 

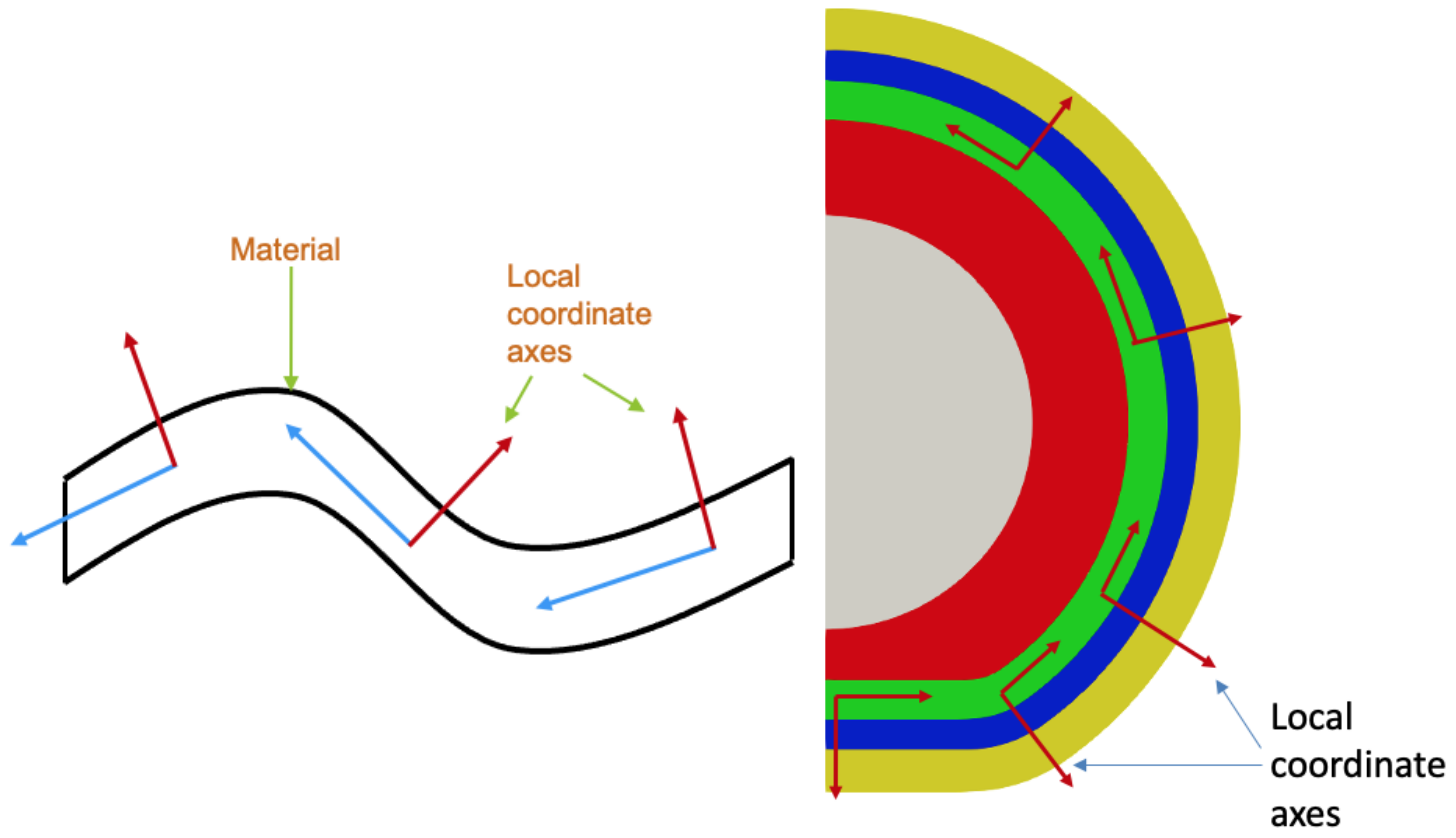

(a) General layered-type geometry

(b) A TRISO particle

Fig. 1. Concept of local coordinate axes in a general layered-type geometry and a TRISO particle.

The local coordinate axes directions at a given quadrature point are determined using the normal vector at that point. The newly implemented algorithm in Bison (using TRISO2DMeshGenerator, NormalVectorsTRISO, and TRISOGeometry) determines the normal vector at any arbitrary point in the particle, irrespective of geometry type (i.e., spherical or aspherical). A transformation matrix is generated using these coordinate axes directions. The transformation matrix is then used to transform the elasticity tensor and the strain tensors from the local coordinate system into the global coordinate system (i.e., Cartesian coordinate system), or vice versa, as needed (BisonUtils). This capability was added to several TRISO material models, such as:

- The PyCCEGAIrradiationEigenstrain material model that computes the irradiation-induced dimensional changes (IIDC) for PyC according to the CEGA

- The PyCIrradiationEigenstrain material model that computes the IIDC for PyC

- The PyCThermalExpansionEigenstrain material model that computes the thermal expansion (per K) and associated eigenstrain for $\mathrm{PyC}$

- The PyCCreepBase base function that computes PyC creep

- The PyCElasticityTensor material model that computes the PyC elasticity tensor.

\section{B. Example Cases}

The benchmark problems described herein are used to demonstrate the improvements to the TRISO capability in Bison. These improvements are shown using the following two example cases: (1) incorporating anisotropy in the elasticity model of PyC (see Section II-B1), and (2) overall improvements in the calculations (see Section II-B2).

1) Incorporating anisotropy in the elasticity model of the PyC layer

The benchmark problem was solved using two types of PyC elasticity models: the isotropic elasticity model and the anisotropic elasticity model. These cases were analyzed for both the spherical and aspherical TRISO particle 
geometries. The following equations show the elastic moduli in the radial and tangential directions for the case of anisotropic elasticity.

The radial and tangential components, $E_{r}$ and $E_{t}$, of the elastic modulus $E(\mathrm{GPa})$ are computed as $[9,10]$ :

$$
\begin{aligned}
E_{r}= & 25.5\left(0.384+0.324 \times 10^{-3} \rho\right)\left(1.463-0.463 B A F_{0}\right) \\
& \times\left(2.985-0.0662 L_{c}\right)(1+0.23 \phi)\left(1+0.00015\left[T_{C}-20\right]\right) \\
E_{t}= & 25.5\left(0.384+0.324 \times 10^{-3} \rho\right)\left(0.481+0.519 B A F_{0}\right) \\
& \times\left(2.985-0.0662 L_{c}\right)(1+0.23 \phi)\left(1+0.00015\left[T_{C}-20\right]\right)
\end{aligned}
$$

where: $\rho \quad=$ the density $\left(\mathrm{kg} / \mathrm{m}^{3}\right)$

$B A F_{0}=$ the as-fabricated anisotropy (-)

$L_{c} \quad=$ the crystallite diameter (30 angstroms)

$\phi \quad=$ the fast neutron fluence $\left(10^{25} \mathrm{n} / \mathrm{m}^{2}, E>0.18 \mathrm{MeV}\right)$

$T_{C} \quad=$ the temperature $\left({ }^{\circ} \mathrm{C}\right)$.

For the isotropic elasticity model, the elastic modulus $E$ is computed as:

$$
E=\frac{E_{r}+2 E_{t}}{3}
$$

The results comparing the maximum radial and tangential stresses and Weibull failure probabilities of the PyC and silicon carbide ( $\mathrm{SiC}$ ) layers for the spherical and aspherical particles are shown in Figures 2-4. The results indicate that the stresses and failure probabilities of the $\mathrm{PyC}$ and the $\mathrm{SiC}$ layers do not differ significantly for spherical particles.

However, for aspherical particles, the stresses and failure probabilities differ significantly. In general, the difference in the stress magnitudes is greatest at either around $1-2 \times 10^{25} \mathrm{n} / \mathrm{m}^{2}$, when the stresses reach their peak value, or toward the end of the analysis. The Weibull failure probabilities for the $\mathrm{PyC}$ and $\mathrm{SiC}$ layers are greater at lower temperatures; the difference in the predicted failure probabilities for these two cases is also greater at lower temperatures. At a temperature of $973 \mathrm{~K}$, the failure probability reaches one (i.e., the PyC layer fails quickly for both cases). These results indicate that the anisotropy in PyC elasticity is important for the aspherical TRISO particle, while for spherical particles, the predicted stresses and failure probabilities are similar to those obtained using the isotropic PyC elasticity model.

2) Overall improvements in the calculations

The benchmark problem was solved using the new TRISO material models, which account for anisotropy and provide the ability to analyze aspherical geometry, as well as using the old TRISO material models. The results comparing the stresses and Weibull failure probability in the $\mathrm{PyC}$ and $\mathrm{SiC}$ layers for these two cases are shown in Figures 5-6.

Figure 5 shows the maximum radial and tangential stresses in the inner PyC layer. The differences in the stress magnitudes obtained from the new and old TRISO models are significant. The difference is greatest at about 1$2 \times 10^{25} \mathrm{n} / \mathrm{m}^{2}$ fluence, when the stresses reach their maximum magnitude, and toward the end of operation at about $5.6 \times 10^{25} \mathrm{n} / \mathrm{m}^{2}$. This difference in the stress magnitudes is significant at all the three temperatures considered in the analyses $(973,1273$, and $1573 \mathrm{~K}$ ). For both radial and tangential stresses, the maximum magnitude difference is about $30-40 \mathrm{MPa}$ at the end of operation for an irradiation temperature of $1573 \mathrm{~K}$.

Figure 5 shows the maximum radial and tangential stresses in the outer PyC layer. As for the inner PyC layer, the stress magnitude differences are significant at all three irradiation temperatures, and the maximum difference in the magnitude of stresses is about $30-40 \mathrm{MPa}$ at the end of operation under the irradiation temperature of $1573 \mathrm{~K}$. For the $\mathrm{SiC}$ layer, the variation of the maximum radial and tangential stresses with fluence is shown in Figure 5. The difference in the stress magnitudes for the SiC layer is lower (i.e., 10-20 MPa) than that for the PyC layers, 


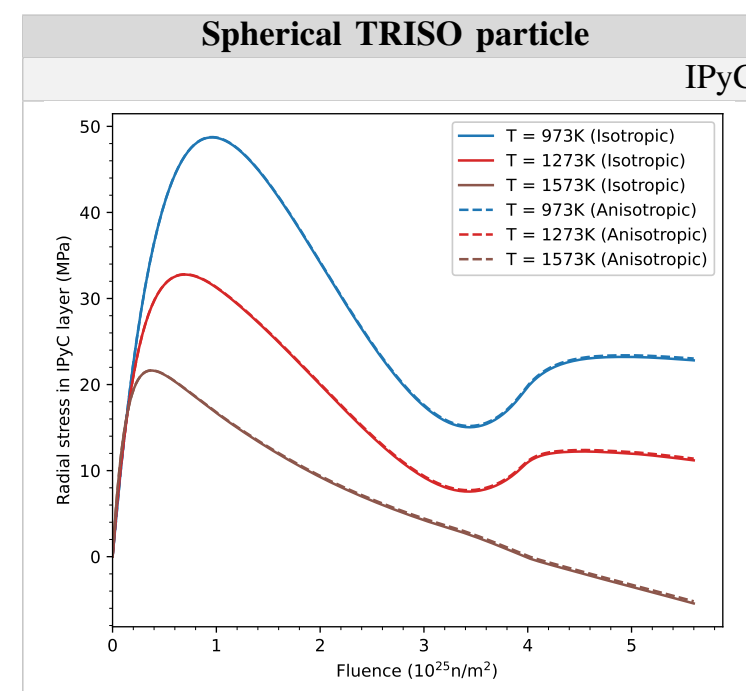

Aspherical TRISO particle

IPyC layer
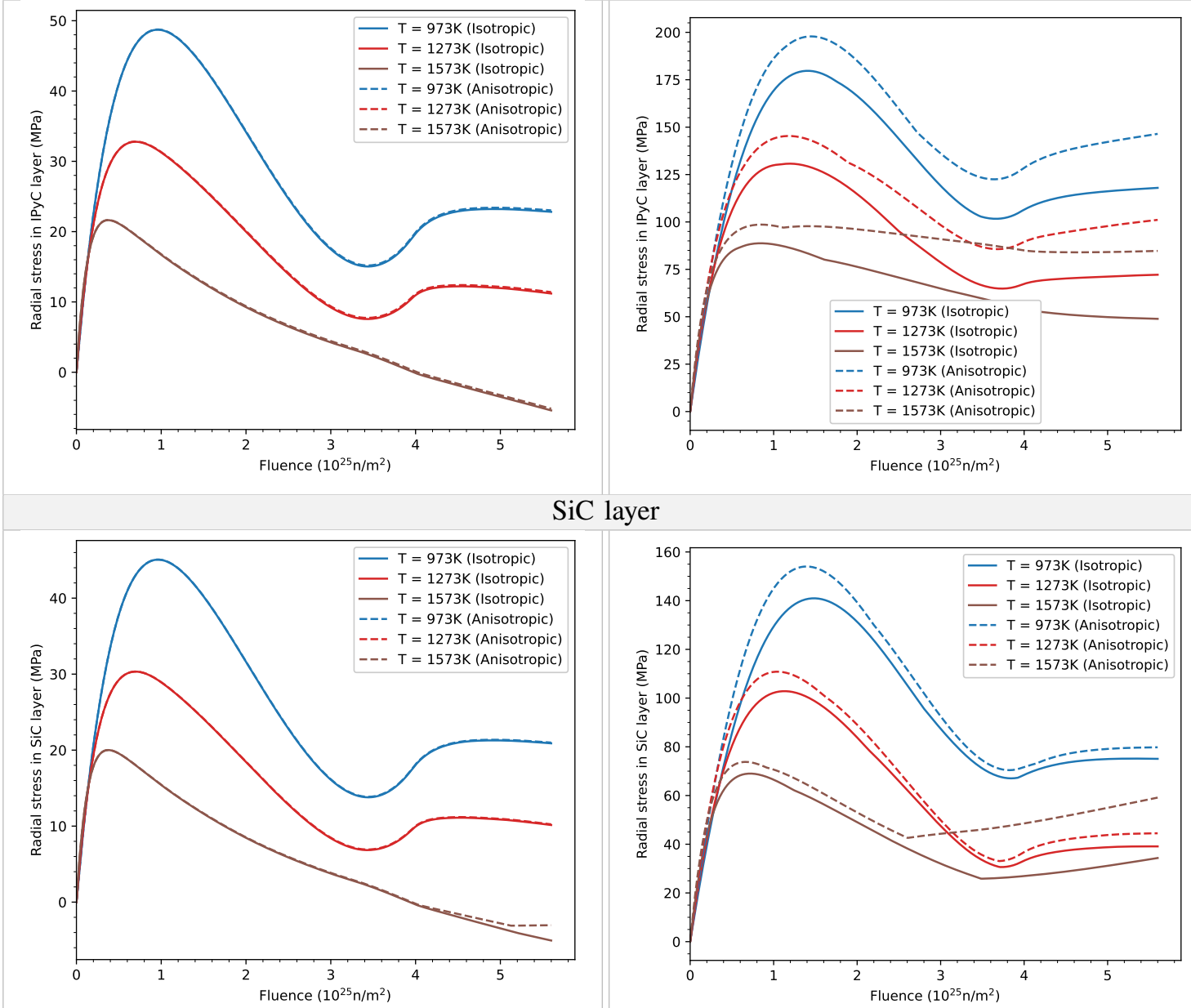

iC layer
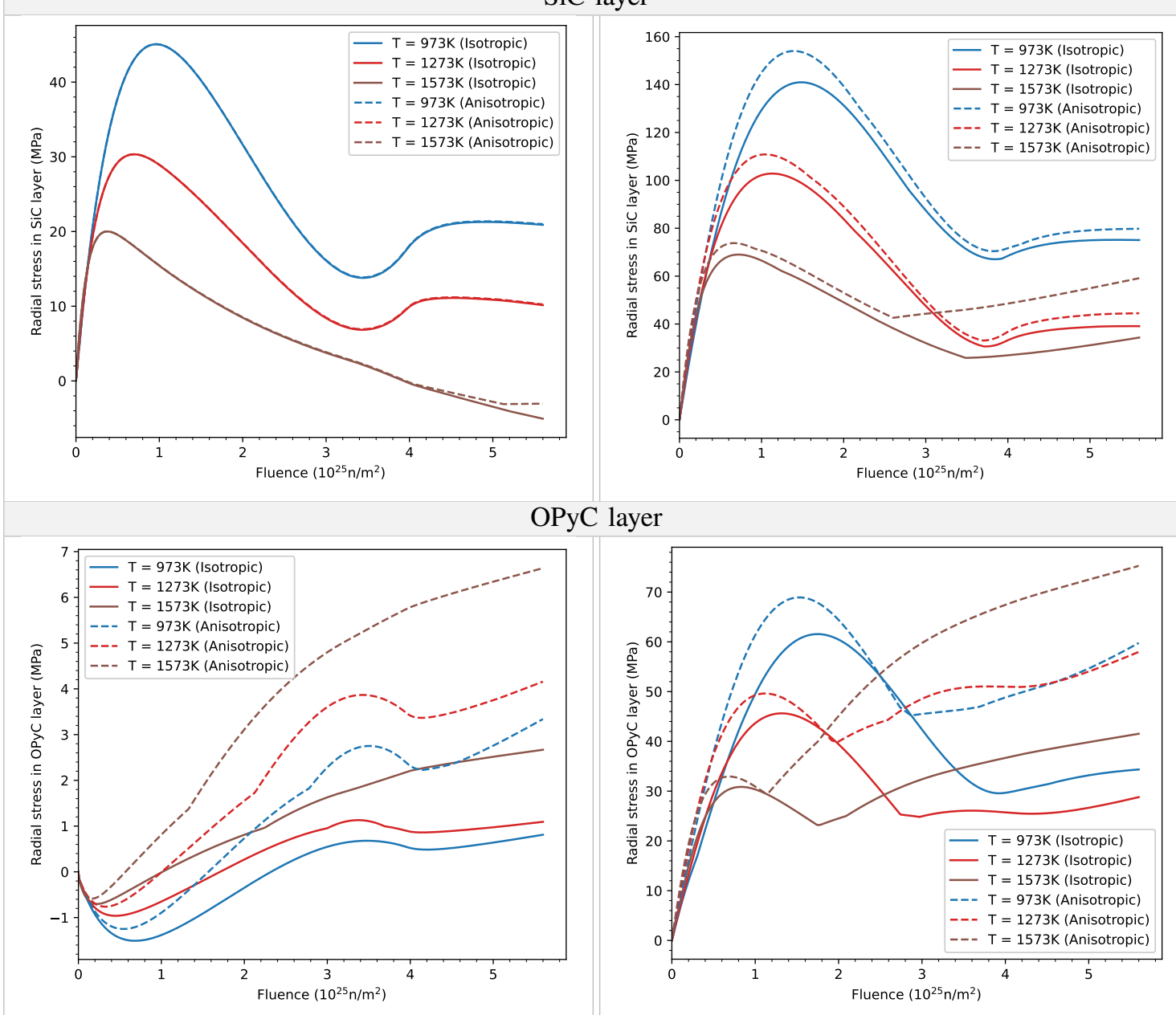

Fig. 2. Variation of maximum radial stress with fluence in the IPyC, $\mathrm{SiC}$, and OPyC layers of a spherical TRISO particle (left column) and an aspherical TRISO particle (right column). 


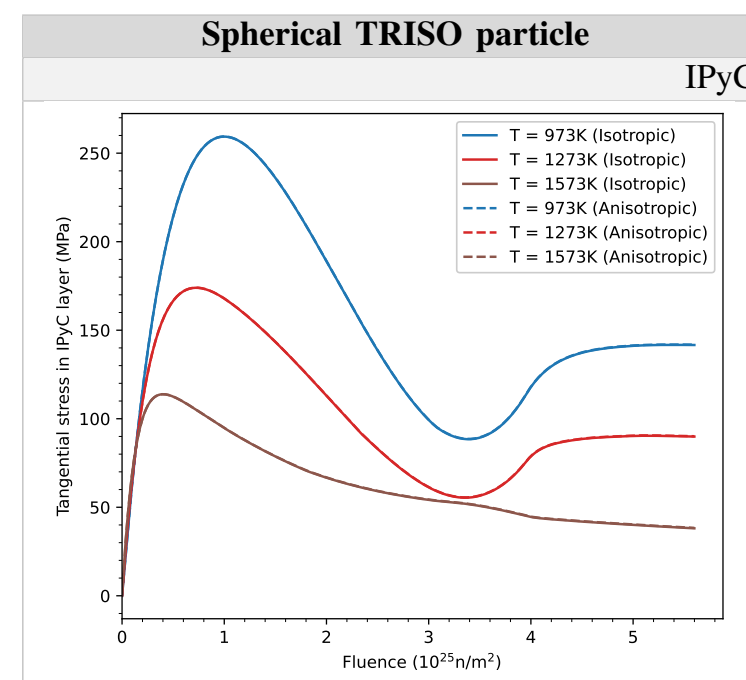

IPyC layer

Aspherical TRISO particle
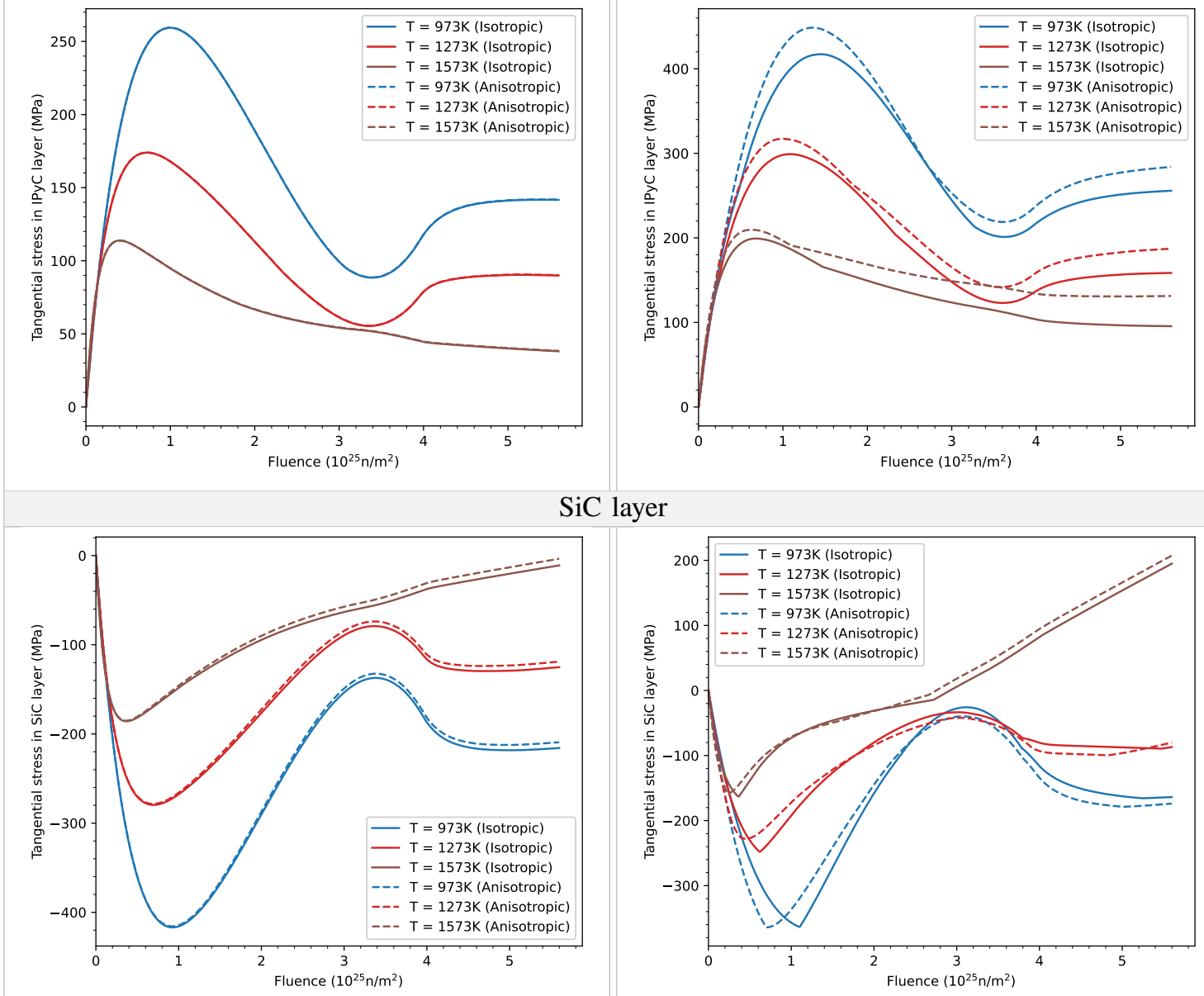

iC layer

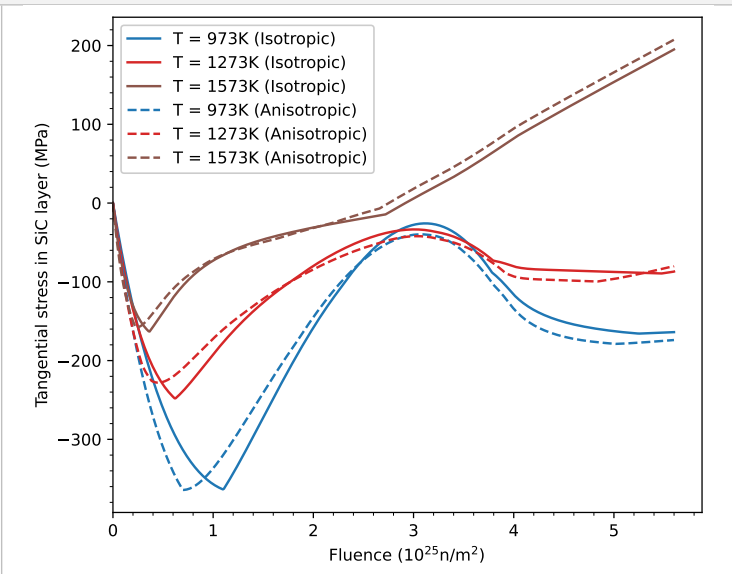

OPyC layer
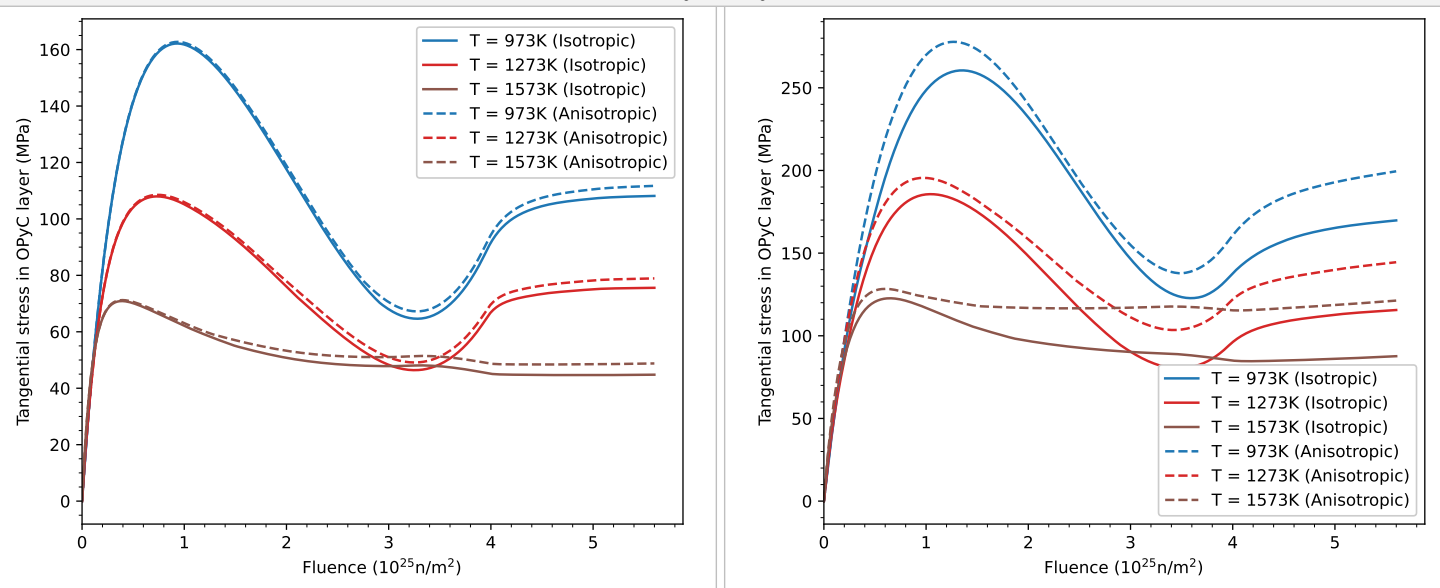

Fig. 3. Variation of maximum tangential stress with fluence in the IPyC, $\mathrm{SiC}$, and $\mathrm{OPyC}$ layers of a spherical TRISO particle (left column) and an aspherical TRISO particle (right column). 


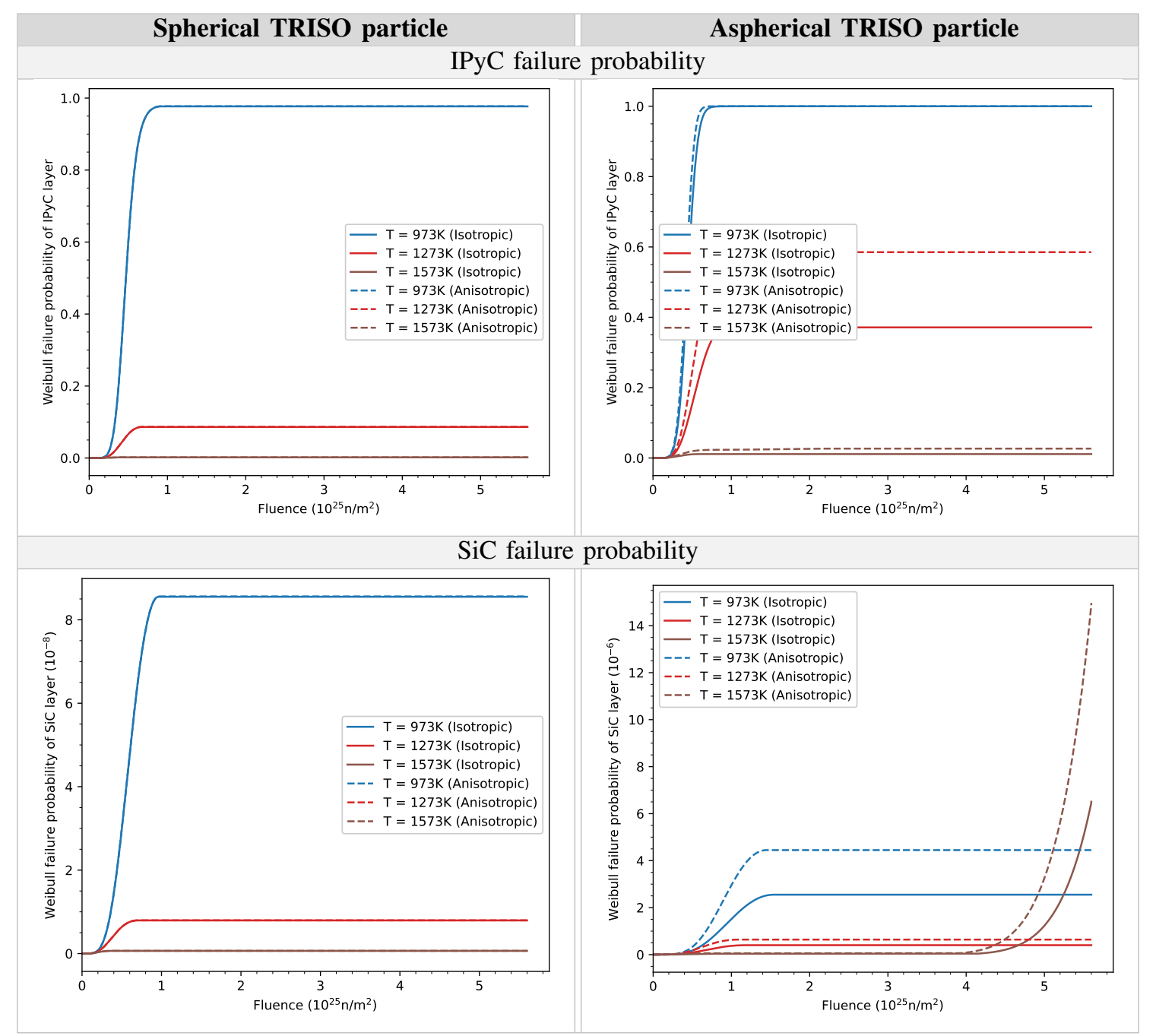

Fig. 4. Variation of failure probability with fluence in the IPyC and $\mathrm{SiC}$ layers of a spherical TRISO particle (left column) and an aspherical TRISO particle (right column).

except for the radial stress, for which the difference in the stress magnitude is greater than $30 \mathrm{MPa}$ at the end of operation under a $1573 \mathrm{~K}$ irradiation temperature.

The calculated failure probability of the inner PyC layer using the new TRISO models is significantly higher than that calculated using old TRISO models at $1273 \mathrm{~K}$, as shown in Figure 6. At the irradiation temperature of $1573 \mathrm{~K}$, the failure probability is low and the difference between the two cases insignificant. However, at $973 \mathrm{~K}$, the failure probability rises steeply to 1.0 for both cases, indicating that the PyC layer has cracked. The Weibull failure probability of the $\mathrm{SiC}$ layer is generally very low $\left(\approx 10^{-6}\right)$. Since the new TRISO model predicts higher tensile stresses in the $\mathrm{SiC}$ layer at all the irradiation temperatures considered, the calculated failure probability is also higher. These results demonstrate that the new TRISO models significantly improve the predictions of TRISO behavior. 




Tangential stress

IPyC layer

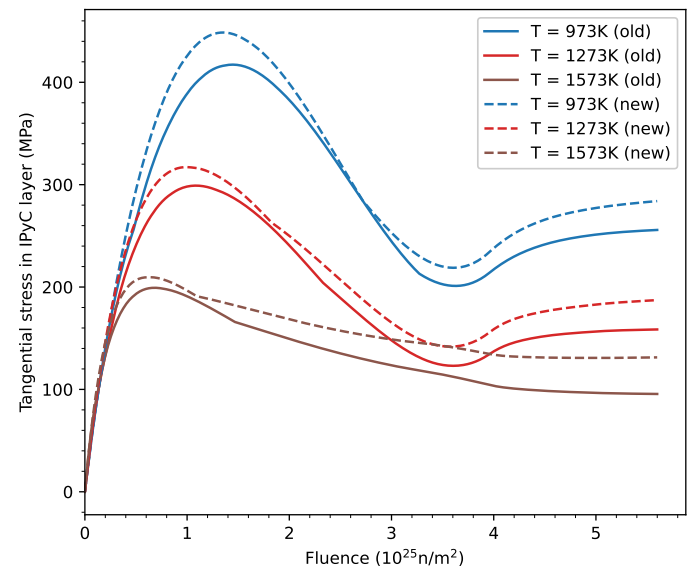

SiC layer
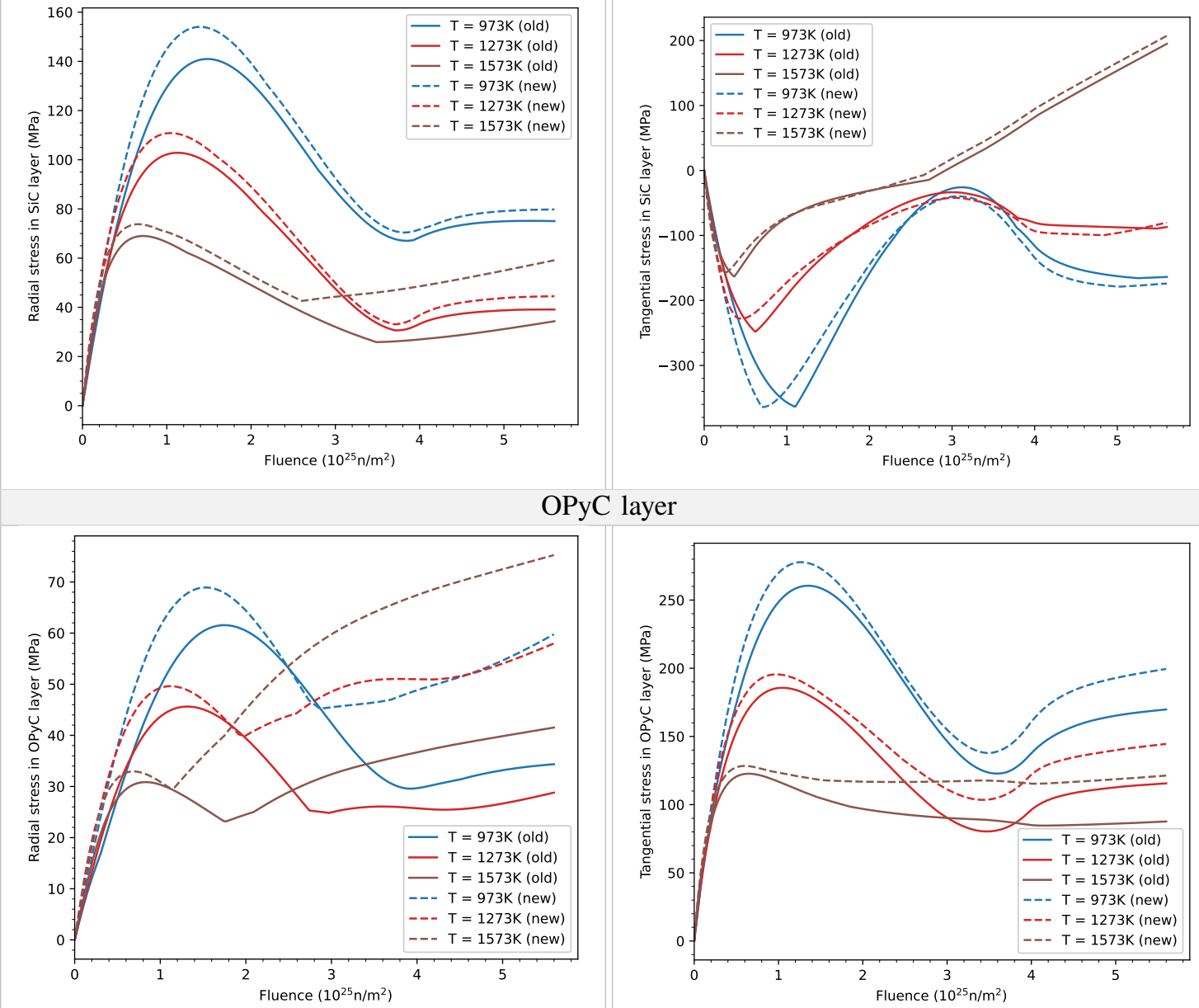

OPyC layer

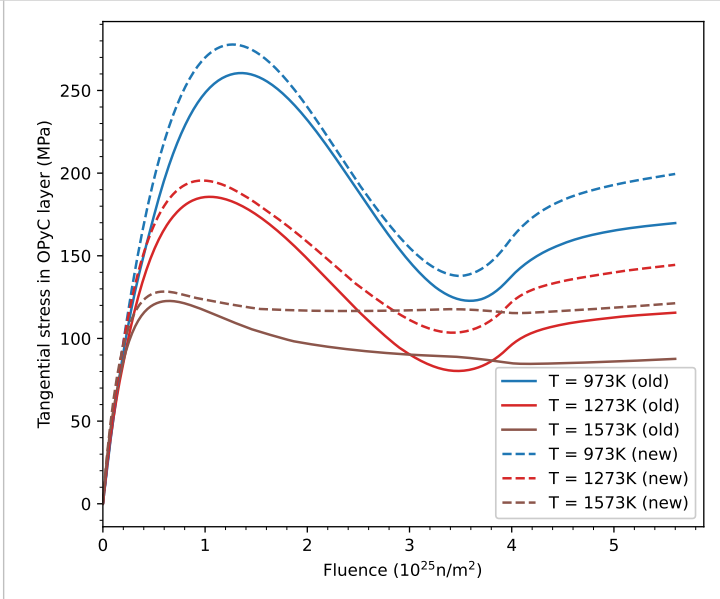

Fig. 5. Variation of maximum radial (left column) and tangential (right column) stresses with fluence in the IPyC, $\mathrm{SiC}$, and OPyC layers. 

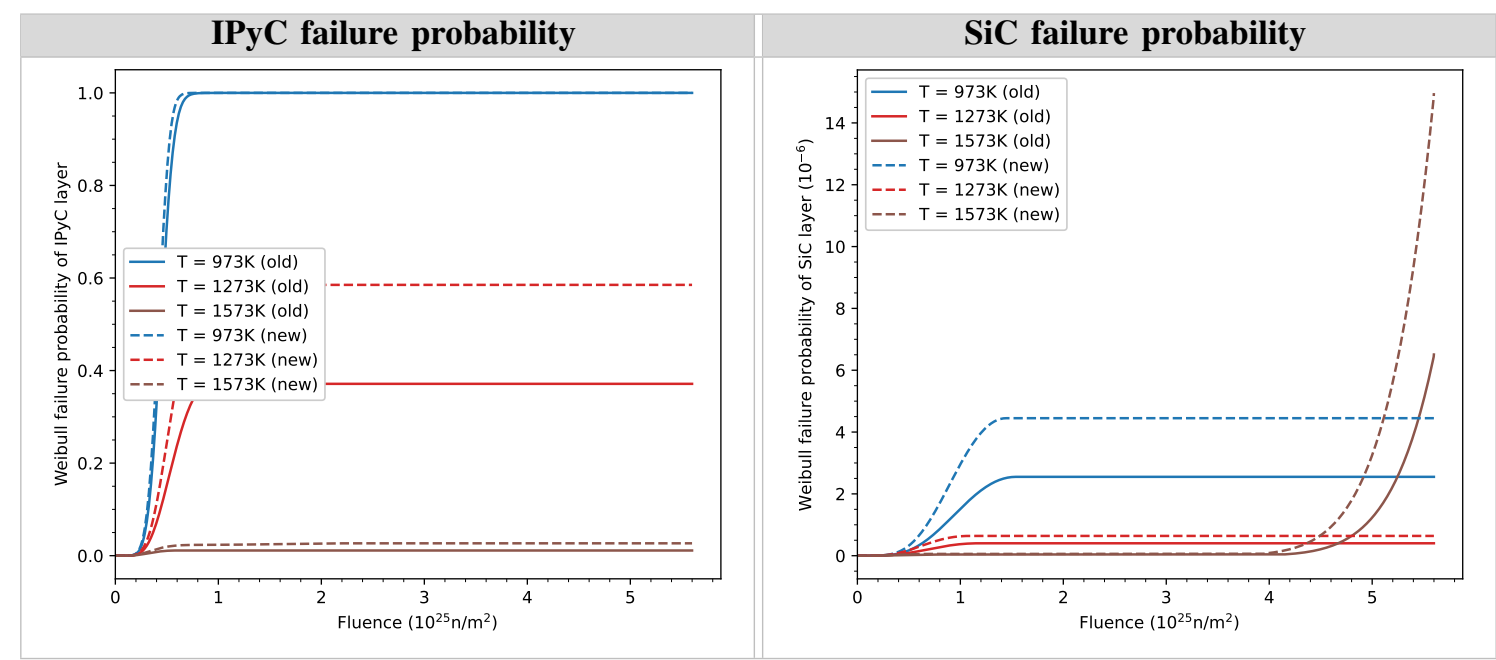

Fig. 6. Variation of failure probability with fluence in the IPyC layer.

\section{Thermal Anisotropy}

The PyC layer can exhibit anisotropy in its thermal properties. It has been reported that the thermal conductivity of $\mathrm{PyC}$ in the radial direction can be significantly lower than that in the tangential direction. This thermal anisotropy in the PyC layer can lead to very different thermal behavior of not only the PyC layer but also of the entire TRISO particle. To incorporate the thermal anisotropy, an anisotropic heat conduction kernel and a general purpose anisotropic heat conduction material that supports the transformation of the thermal conductivity tensor from one coordinate system to another was implemented in Multiphysics Object-Oriented Simulation Environment (MOOSE) (in the AnisoHeatConduction kernel and AnisoHeatConductionMaterial material model). Future work will involve developing an anisotropic PyC thermal model in Bison, based on these MOOSE models. 


\section{Modeling Debonding In TRISO Fuel Particles}

Debonding of TRISO layers and the associated effects on the mechanical failure of TRISO particles is experimentally evident in recent AGR-1 [11] and AGR-2 [12] experiments. Buffers that are entirely debonded from the IPyC layer are typically observed in experiments, due to irradiation-induced shrinkage, and are not likely to cause cracking of the IPyC and SiC layers. Partial debonding at the buffer-IPyC interface results in stress concentrations in buffers, sometimes causing the IPyC layer to crack. Delamination at the IPyC-SiC interface was observed, potentially resulting in radial cracks in the $\mathrm{SiC}$ layer [13]. Debonding of the outer PyC (OPyC) may also occur during irradiation as the matrix shrinks away from the particles and pulls outward on the OPyC layer. Such experimental evidence highlights the importance of developing a capability in Bison to model TRISO layer interface debonding. Cohesive zone modeling (CZM) is a powerful tool for predicting delamination in adhesively bonded structures, and its framework was implemented in MOOSE [14]. This section summarizes new code development for the CZM and demonstrates several numerical examples of TRISO debonding.

\section{A. Mesh Modifier for Creating Cohesive Zone Interfaces}

A new type of block restricted option was added in MOOSE's BreakMeshByBlockGenerator. The new block_pairs option will only create interfaces between the specified block pairs. In addition, the add_interface_on_two_sides parameter enables interface boundaries to be generated on both sides of the interface, and these boundaries can be used to enforce thermal and mechanical contact at the interface.

An example of the mesh modifier is shown in Figure 7. The original mesh has three fully connected blocks. By using the block_pairs = '2 3 ' option, a CZM interface boundary between blocks 2 and 3 is created by appropriately duplicating nodes between the two blocks. With the add_interface_on_two_sides = true option, a second interface boundary is added on the other side of block 3 . These two interface boundaries can be used as primary and secondary surfaces in MOOSE's thermal and mechanical constraints.

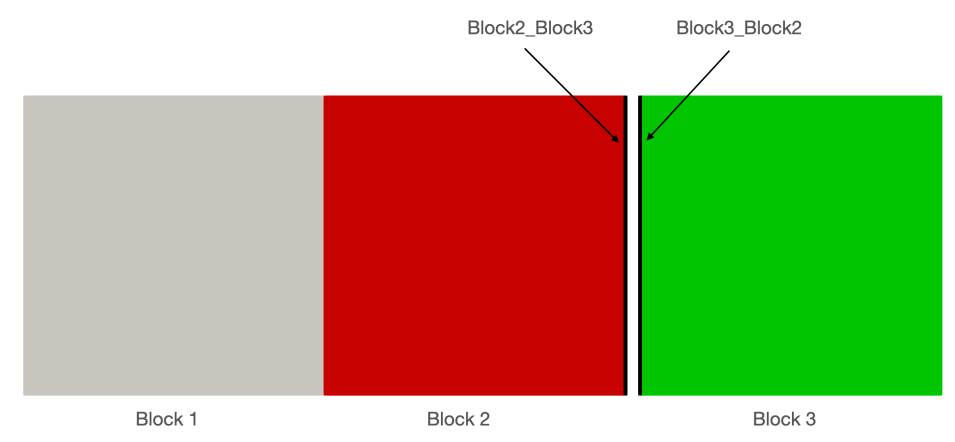

Fig. 7. Three-block example: two interface boundaries are created between block 2 and block 3, named "Block2_Block3" and "Block3_Block2", respectively.

\section{B. Bilinear Mixed-Mode Traction Separation Law}

The bilinear mixed mode traction separation law described in [15] was implemented in MOOSE to model the initiation and propagation of debonding between TRISO layers. The model description is outlined below. 


\section{1) Softening onset prediction}

Initiation of the softening process is predicted using the following quadratic failure criterion:

$$
\left(\frac{\left\langle\tau_{3}\right\rangle}{N}\right)^{2}+\left(\frac{\tau_{2}}{S}\right)^{2}+\left(\frac{\tau_{1}}{T}\right)^{2}=1
$$

where: $N=$ interlaminar tensile strength

$S$ and $T=$ interlaminar shear strengths

$\tau_{i} \quad=$ local traction

The Macaulay bracket $\langle\cdot\rangle$ is used to describe the ramp function:

$$
\langle x\rangle=\left\{\begin{array}{l}
0, x>0 \\
x, x \geq 0
\end{array}\right.
$$

The total mixed-mode relative displacement $\delta_{m}$ is defined as:

$$
\delta_{m}=\sqrt{\delta_{1}^{2}+\delta_{2}^{2}+\left\langle\delta_{3}\right\rangle^{2}}=\sqrt{\delta_{\text {shear }}^{2}+\left\langle\delta_{3}\right\rangle^{2}}
$$

where: $\quad \delta_{\text {shear }}=$ the norm of the vector defining the tangential relative displacements of the element.

Using the same penalty stiffness in Modes I, II, and III, the tractions before softening onset are:

$$
\tau_{i}=K \delta_{i}
$$

for $i=1,2,3$.

Assuming $S=T$, the single-mode relative displacements at the onset of softening are:

$$
\delta_{3}^{0}=\frac{N}{K}
$$

and

$$
\delta_{1}^{0}=\delta_{2}^{0}=\delta_{\text {shear }}^{0}=\frac{S}{K}
$$

For an opening displacement $\delta_{3}$ greater than zero, the mode mixity ratio $\beta$ is defined as:

$$
\beta=\frac{\delta_{\text {shear }}}{\delta_{3}}
$$

The mixed-mode relative displacement corresponding to the onset of softening $\delta_{m}^{0}$ is given as:

$$
\delta_{m}^{0}= \begin{cases}\delta_{3}^{0} \delta_{1}^{0} \sqrt{\frac{1+\beta^{2}}{\left(\delta_{1}^{0}\right)^{2}+\left(\beta \delta_{3}^{0}\right)^{2}}} & \delta_{3}>0 \\ \delta_{\text {shear }}^{0} & \delta_{3} \leq 0\end{cases}
$$

2) Delamination propagation prediction

Two types of propagation criterion were implemented in MOOSE: (1) a power-law criterion, and (2) a Benzeggagh and Kenane (B-K) criterion. The mathematical formulations of each criterion are detailed below.

1) The power law criterion is given as:

$$
\left(\frac{G_{I}}{G_{I C}}\right)^{\alpha}+\left(\frac{G_{I I}}{G_{I I C}}\right)^{\alpha}=1
$$


The mixed-mode displacements corresponding to total decohesion are given as:

$$
\delta_{m}^{f}= \begin{cases}\frac{2\left(1+\beta^{2}\right)}{K \delta_{m}^{0}}\left[\left(\frac{1}{G_{I C}}\right)^{\alpha}+\left(\frac{\beta^{2}}{G_{I I C}}\right)^{\alpha}\right]^{-1 / \alpha} & \delta_{3}>0 \\ \sqrt{\left(\delta_{1}^{f}\right)^{2}+\left(\delta_{2}^{f}\right)^{2}} & \delta_{3} \leq 0\end{cases}
$$

2) The B-K criterion, proposed by Benzeggagh and Kenane [16], is given as:

$$
G_{I C}+\left(G_{I I C}-G_{I C}\right)\left(\frac{G_{\text {shear }}}{G_{T}}\right)^{\eta}=G_{C}
$$

with

$$
G_{T}=G_{I}+G_{\text {shear }}
$$

The mixed-mode displacements corresponding to total decohesion are given as:

$$
\delta_{m}^{f}= \begin{cases}\frac{2}{K \delta_{m}^{0}}\left\{G_{I C}+\left(G_{I I C}-G_{I C}\left[\frac{\beta^{2}}{1+\beta^{2}}\right]^{\eta}\right)\right\} & \delta_{3}>0 \\ \sqrt{\left(\delta_{1}^{f}\right)^{2}+\left(\delta_{2}^{f}\right)^{2}} & \delta_{3} \leq 0\end{cases}
$$

3) Constitutive equation for mixed-mode loading

The constitutive equation for mixed-mode loading is given as:

$$
\begin{gathered}
\tau_{s}=D_{s r} \delta_{r} \\
D_{s r}= \begin{cases}\bar{\delta}_{s r} K & \text { if } \delta_{m}^{\max } \leq \delta_{m}^{0} \\
\bar{\delta}_{s r}\left[(1-d) K+K d \bar{\delta}_{s 3} \frac{\left\langle-\delta_{3}\right\rangle}{-\delta_{3}}\right] & \text { if } \delta_{m}^{0}<\delta_{m}^{\max } \leq \delta_{m}^{f} \\
\bar{\delta}_{s 3} \bar{\delta}_{3 r} \frac{\left\langle-\delta_{3}\right\rangle}{-\delta_{3}} K & \text { if } \delta_{m}^{\max } \geq \delta_{m}^{f}\end{cases} \\
\quad d=\frac{\delta_{m}^{f}\left(\delta_{m}^{\max }-\delta_{m}^{0}\right)}{\delta_{m}^{\max }\left(\delta_{m}^{f}-\delta_{m}^{0}\right)} \quad d \in[0,1]
\end{gathered}
$$

\section{4) Viscous regularization}

Cohesive zone models that exhibit softening behavior and stiffness degradation often lead to convergence difficulties in an implicit solver. The traction separation laws can be regularized using viscosity. The viscous damage variable $d_{v}$ is defined by:

$$
\dot{d}_{v}=\frac{1}{\mu}\left(d-d_{v}\right)
$$

where $\mu$ is the viscosity parameter representing the relaxation time of the viscous system. An analytical expression of $d_{v}$ can be obtained using the backward Euler method. With viscous regularization, the $d$ will be replaced by $d_{v}$ in Equation 15b to compute traction.

\section{Thermal Heat Transfer between Debonded Layers}

Heat transfer across debonded layers is modeled using the following relation [17], neglecting the contribution of the solid contact conductance:

$$
h_{\text {gap }}=h_{g}+h_{r}
$$


where: $\quad h_{\text {gap }}=$ the total conductance across the gap

$h_{g}=$ the gas conductance

$h_{r} \quad=$ the conductance due to radiant heat transfer.

The fill gas conductance, $h_{g}$, is described as follows, assuming the continuum flow:

$$
h_{g}=\frac{k_{g}}{d_{g}}
$$

where: $k_{g}=$ the conductivity of the gas in the gap

$d_{g}=$ is the gap width.

The conductance due to radiant heat transfer, $h_{r}$, is computed as follows [18]:

$$
h_{r}=\frac{\sigma\left(T_{1}^{2}+T_{2}^{2}\right)\left(T_{1}+T_{2}\right)}{1 / \epsilon_{1}+1 / \epsilon_{2}-1}
$$

where: $\sigma=$ the Stefan-Boltzmann constant

$T_{1}, T_{2}=$ the temperatures of the radiating surfaces

$\epsilon_{1}, \epsilon_{2}=$ the emissivities of the surrounding radiating surfaces.

A two-block example was used to verify whether the thermal contact can be correctly enforced on the CZM interfaces. In this example, two interface boundaries were created by BreakMeshByBlockGenerator. The temperatures on the left and right boundary were set to 100 and $0 \mathrm{~K}$, respectively. The two blocks were separated by a gap of $0.1 \mathrm{~m}$. The thermal contact constraint was applied to transfer the heat from one block to the other. Four different gap conductivity values (i.e., $0.01,0.1,1$, and $10 \mathrm{~W} / \mathrm{m}-\mathrm{K}$ ) were considered. The temperature contours are shown in Figure 8. As the gap conductivity increases, more heat can be transferred from the left block to the right block. As shown in Figure 9, the temperature jump across the gap decreases as the gap conductivity increases. For a gap conductivity of $10 \mathrm{~W} /(\mathrm{m}-\mathrm{K})$, the temperature is nearly continuous across the two blocks.

\section{TRISO Layer Debonding Examples}

In this section, the power-law propagation criterion was used as the criterion for delamination propagation. The CZM properties used in all the simulations are provided in Table I. For heat transfer across debonded layers, $h_{g}$ was taken as $100 \mathrm{~W} /(\mathrm{m}-\mathrm{K})$ and $\epsilon_{1}=\epsilon_{2}=1(-)$. The fuel properties and simulation conditions are listed in Section IV-E. Condition 2-with an irradiation temperature of $973 \mathrm{~K}$-was simulated for this report.

TABLE I. Simulation settings for the CZM properties.

\begin{tabular}{|cl|ll|ll|ll|}
\hline $\mathrm{G}_{I C}$ & $8 \mathrm{~J} / \mathrm{m}^{2}$ & $\mathrm{~K}$ & $4 \times 10^{14} \mathrm{~N} / \mathrm{m}$ & $\mathrm{S}$ & $10 \mathrm{MPa}$ & $\mu$ & 1 second \\
\hline $\mathrm{G}_{I I C}$ & $4 \mathrm{~J} / \mathrm{m}^{2}$ & $\mathrm{~N}$ & $30 \mathrm{MPa}$ & $\alpha$ & 2 & & \\
\hline
\end{tabular}

To allow debonding to initiate at any arbitrary point, a scale factor with random values between 1.0 and 1.1 was applied to the normal strength, N. The random factor field is shown in Figure 10. The random factor values are generated for all elements but are used only at the interface where the CZM applies.

1) Debonding of the IPyC from the $\mathrm{SiC}$

During irradiation, shrinkage of the IPyC layer induces a radial tensile stress at the interface between the IPyC and $\mathrm{SiC}$ layers. As shown in Figure 12, when the radial stress reaches a bond strength of $30 \mathrm{MPa}$, debonding of the IPyC from the SiC occurs. In this simulation, debonding initiates from the center, where the randomized normal strength is the weakest. During irradiation, it progressively propagates along the interface toward the two ends. It can be seen from Figure 13 that a stress concentration occurs in the SiC layer at the tip of the debonding region. 

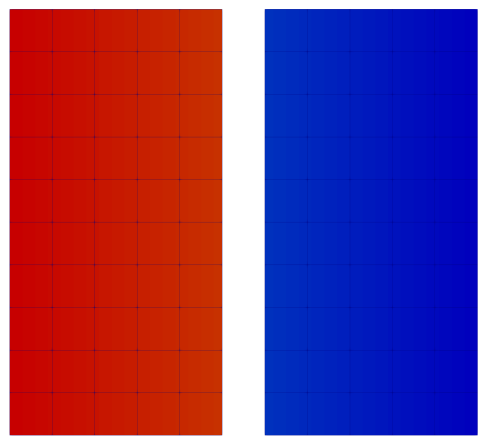

(a) $k_{g}=0.01$
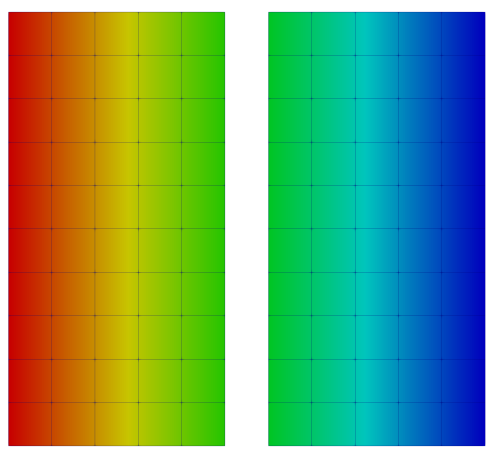

(c) $k_{g}=1.0$
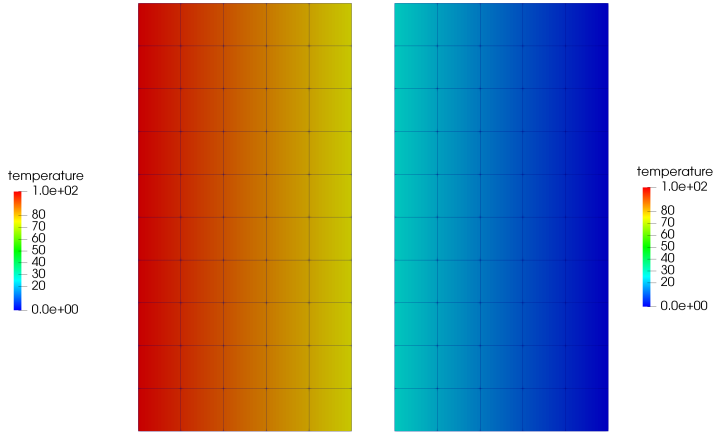

(b) $k_{g}=0.1$
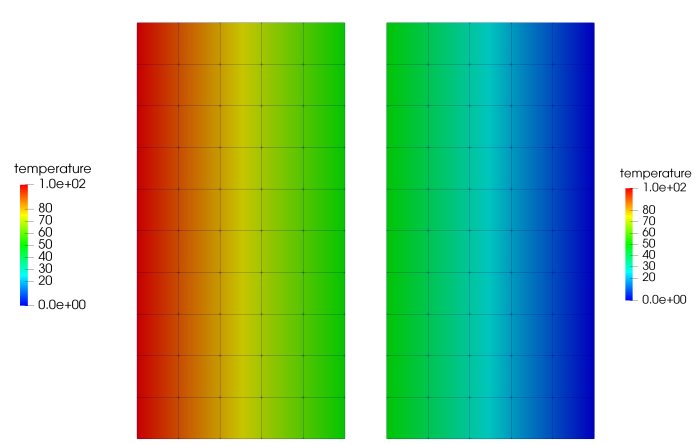

(d) $k_{g}=10.0$

Fig. 8. Temperature contour plots with varying fill gas conductivity from the two-block verification example.

Although this stress is compressive under this simulation condition, it might become tensile and cause failure of the $\mathrm{SiC}$ layer when irradiation conditions change. The damage evolution is shown in Figure 11. At the end of irradiation, the interface is fully debonded at the bottom, yet remains connected at the top. The time history of stress in the $\mathrm{SiC}$ layer during debonding of the $\mathrm{IPyC}$ from the $\mathrm{SiC}$ is shown in Figure 14.

Next, debonding of the IPyC from the SiC layer was considered for an aspherical particle. A single flat facet created during fabrication on one side of the particle is a common cause of aspherical behavior in particle fuel. In Figure 15, it is interesting to see that the debonding initiates at the corner where a radial stress concentration occurs. This is different from the spherical particle case, in which the radial stress is uniform at the interface and the debonding initiates at the weakest strength point.

2) Debonding of the buffer from the IPyC

As the buffer layer shrinks under irradiation, a gap can form between the buffer and IPyC layer. Three cases were considered in this regard: (1) the buffer and IPyC are fully debonded at initial time, (2) debonding between the buffer and the IPyC with a bond strength of $30 \mathrm{MPa}$, and (3) debonding between the buffer and the IPyC with a bond strength of $80 \mathrm{MPa}$. The damage evolution and radial stress of debonding between the buffer and IPyC layers is shown in Figure 17 and Figure 20, respectively. In this simulation, the debonding initiates near the bottom of the interface and then propagates, fully separating the two layers. Due to the large shrinkage of the buffer layer, the radial stress is high, causing debonding to occur in the early irradiation stage. As seen from Figure 18, the radial stress reaches bond strength in a very early stage. The debonding produces a stress concentration in the tangential 


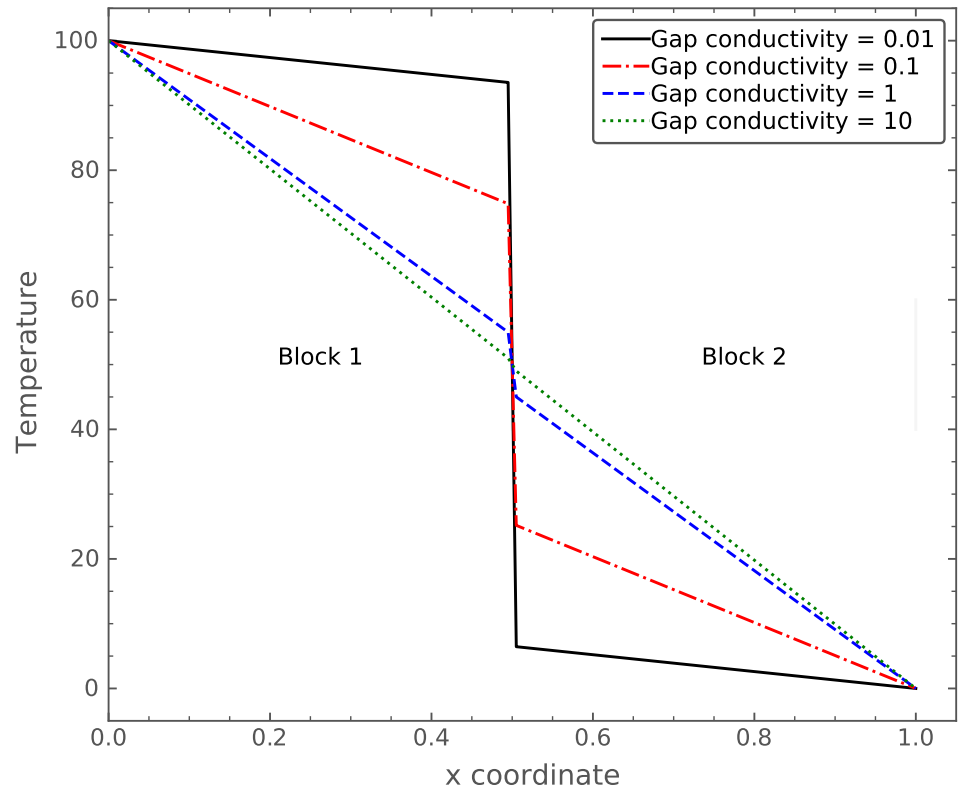

Fig. 9. Temperature predictions across the center horizontal line from the two-block verification example.

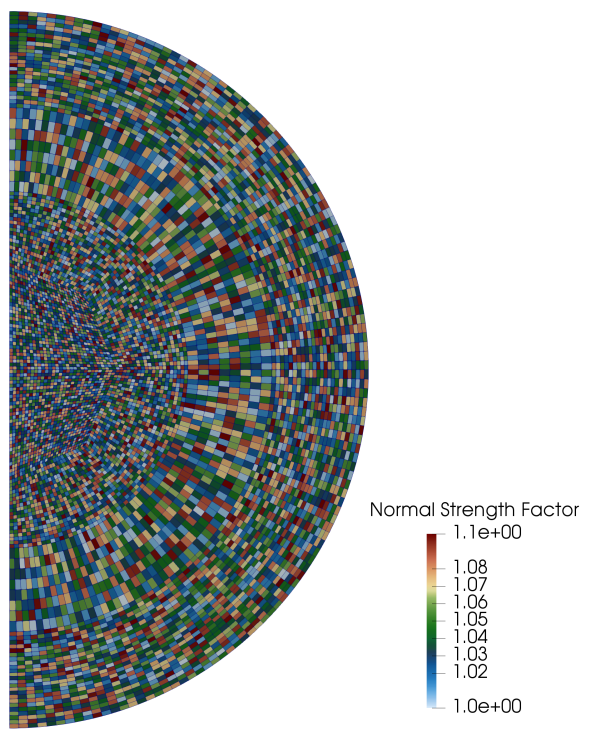

Fig. 10. Random scale factor for normal strength.

direction. After fully debonding, the stress remains the same as that in the case involving full initial debonding.

Fractures in irradiated IPyC layers were observed in AGR-1 experiments, suggesting that the IPyC layer could be torn during buffer-IPyC debonding at interfacial points where bonding was especially strong. The IPyC fractures were found immediately adjacent to the circumferential region that still had a bonded buffer-IPyC interface. An 


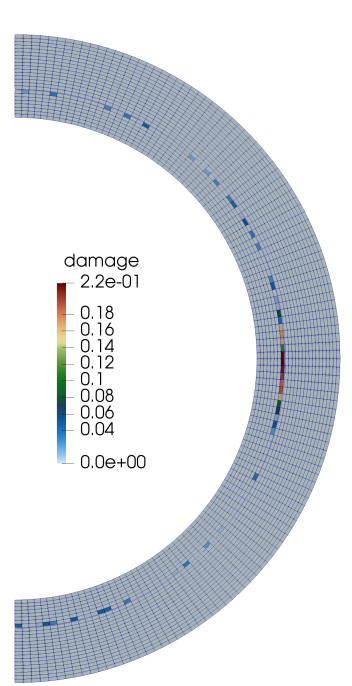

(a) $t=2654616 \mathrm{~s}$



(b) $t=2654622 \mathrm{~s}$

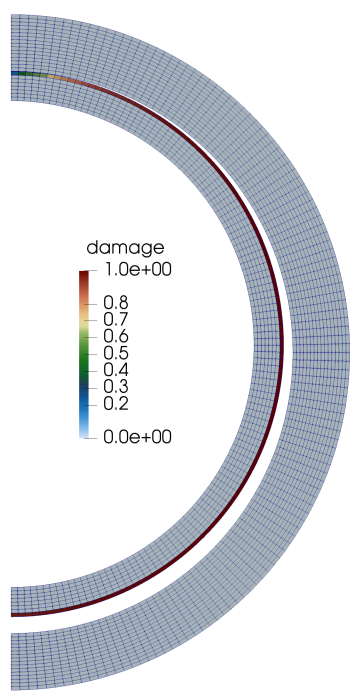

(c) $t=2654627 \mathrm{~s}$

Fig. 11. Damage evolution during debonding of the IPyC from the SiC at $t=$ (a) $2654616 \mathrm{~s}$, (b) $2654622 \mathrm{~s}$, and (c) $2654627 \mathrm{~s}$ (displacements are magnified 10x).

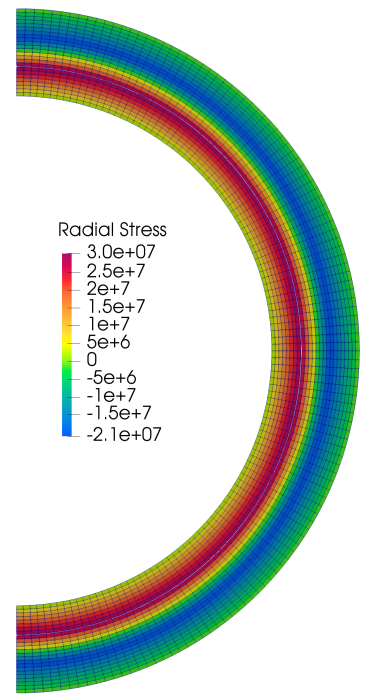

(a) $t=2654616 \mathrm{~s}$

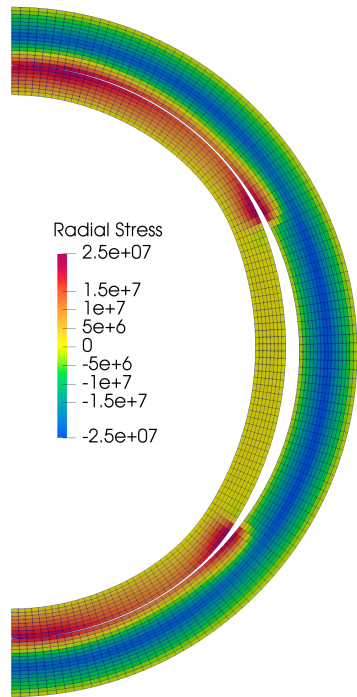

(b) $t=2654622 \mathrm{~s}$

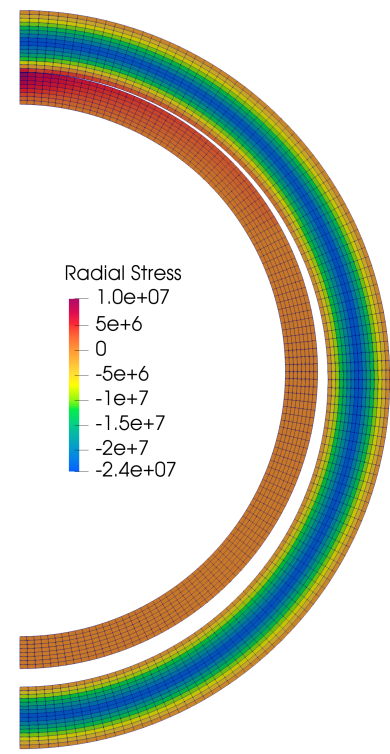

(c) $t=2654627 \mathrm{~s}$

Fig. 12. Radial stress in the IPyC, $\mathrm{SiC}$, and $\mathrm{OPyC}$ layers during debonding of the $\mathrm{IPyC}$ from the $\mathrm{SiC}$ at $t=(\mathrm{a})$ $2654616 \mathrm{~s}$, (b) $2654622 \mathrm{~s}$, and (c) $2654627 \mathrm{~s}$ (displacements are magnified 10x). 


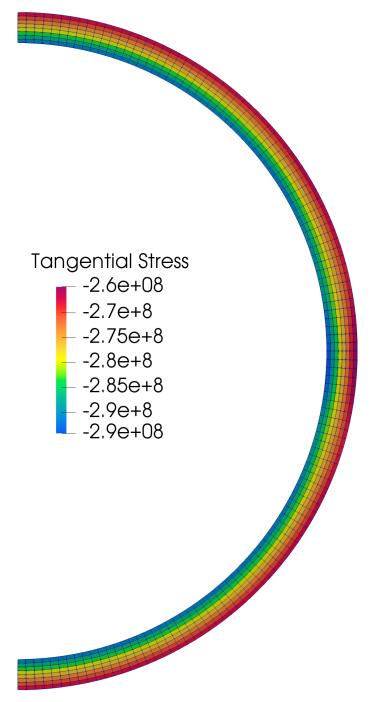

(a) $t=2654616 \mathrm{~s}$

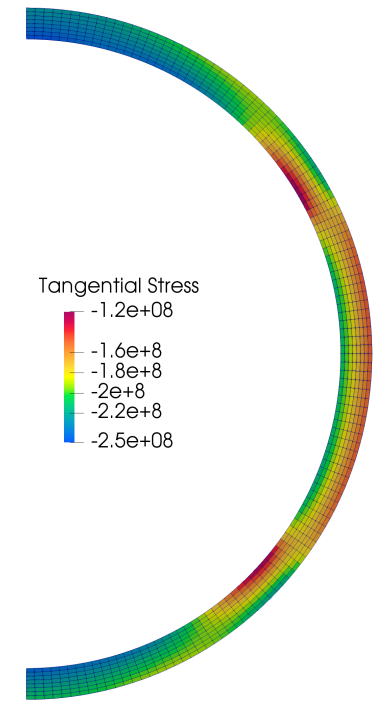

(b) $t=2654622 \mathrm{~s}$

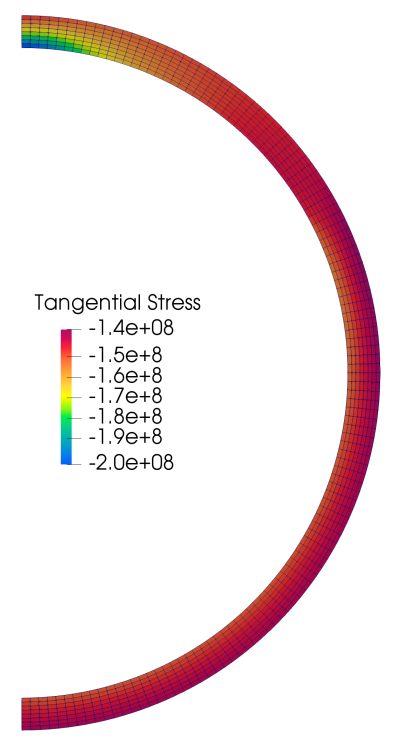

(c) $t=2654627 \mathrm{~s}$

Fig. 13. Tangential stress in the SiC layer during debonding of the IPyC from the SiC at $t=$ (a) $2654616 \mathrm{~s}$, (b) $2654622 \mathrm{~s}$, and (c) $2654627 \mathrm{~s}$.

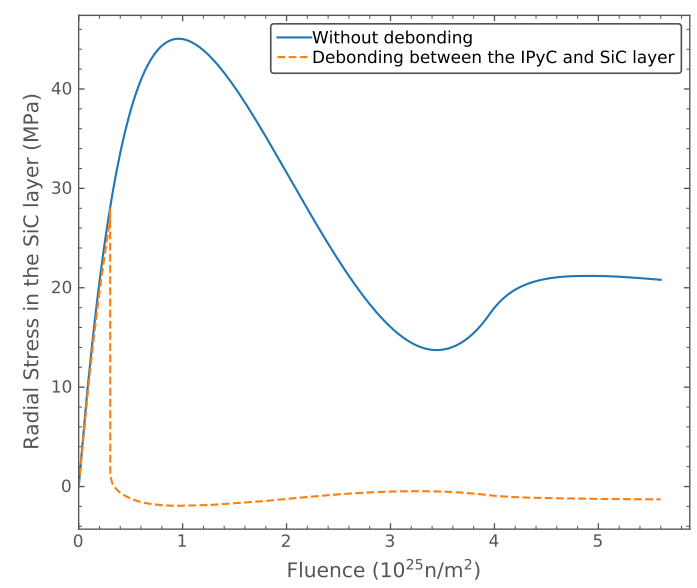

(a) Radial stress

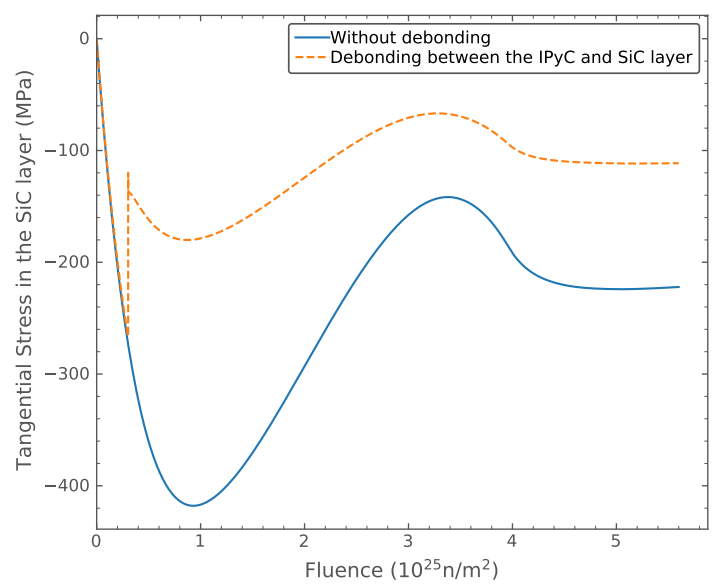

(b) Tangential stress

Fig. 14. Time history of stress in the $\mathrm{SiC}$ layer during debonding of the IPyC from the SiC. 


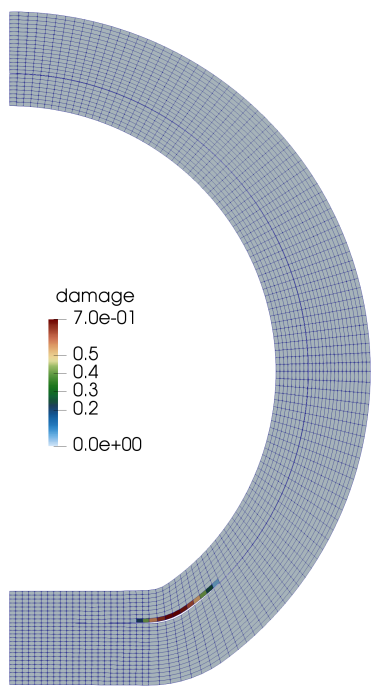

(a) $t=1555200 \mathrm{~s}$

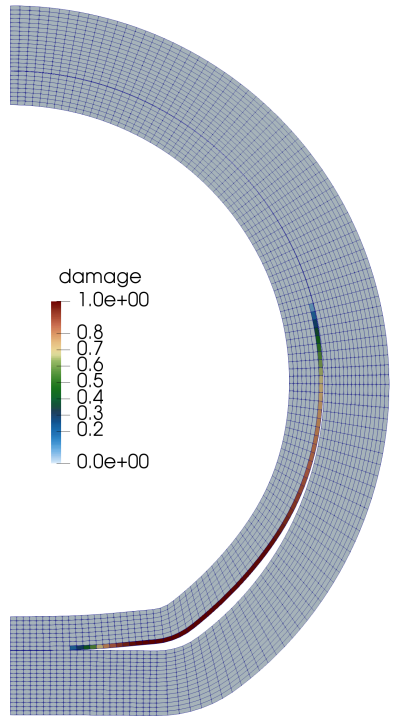

(b) $t=1614426 \mathrm{~s}$

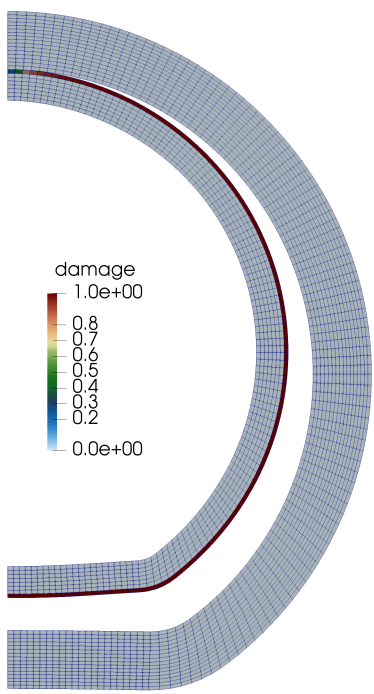

(c) $t=4707012 \mathrm{~s}$

Fig. 15. Damage evolution during debonding of the IPyC from the SiC in an aspherical particle at $t=$ (a) $1555200 \mathrm{~s}$, (b) $1614426 \mathrm{~s}$, and (c) $4707012 \mathrm{~s}$ (displacements are magnified 10x).

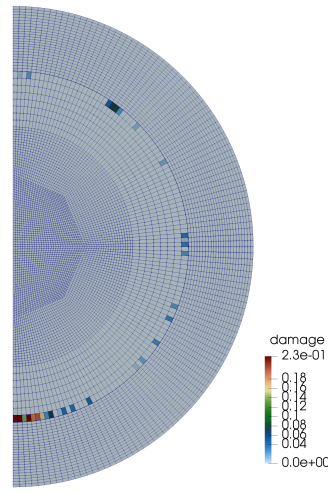

(a) $t=612481 \mathrm{~s}$



(b) $t=612491 \mathrm{~s}$

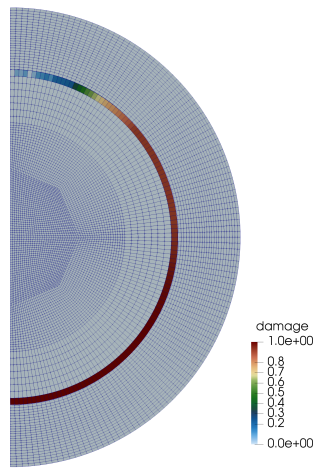

(c) $t=612502 \mathrm{~s}$

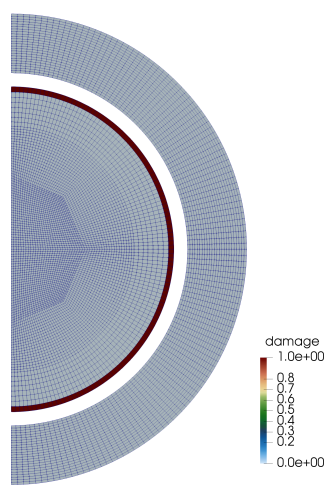

(d) $t=48313150 \mathrm{~s}$

Fig. 16. Damage evolution during debonding of the buffer from the IPyC with a bond strength of $80 \mathrm{MPa}$ at $t=$ (a) $612481 \mathrm{~s}$, (b) $612491 \mathrm{~s}$, (c) $612502 \mathrm{~s}$, and (d) $48313150 \mathrm{~s}$. 


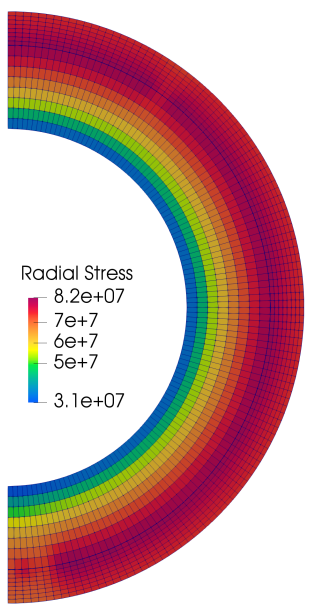

(a) $t=612481 \mathrm{~s}$

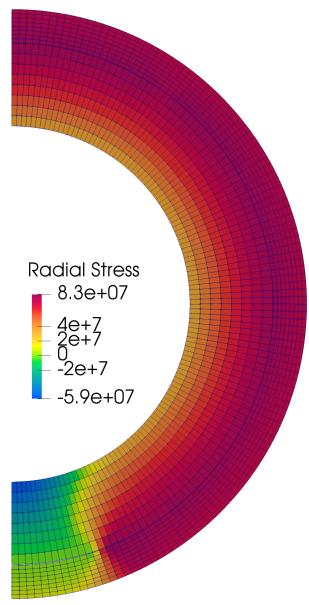

(b) $t=612491 \mathrm{~s}$

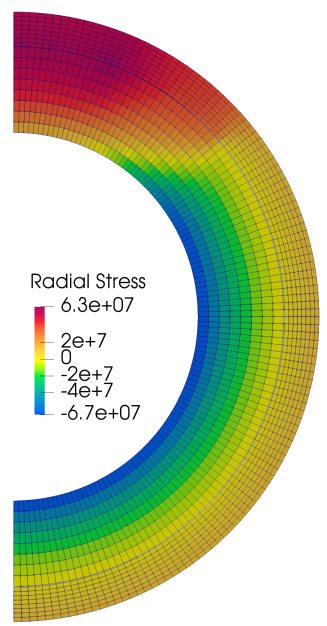

(c) $t=612502 \mathrm{~s}$

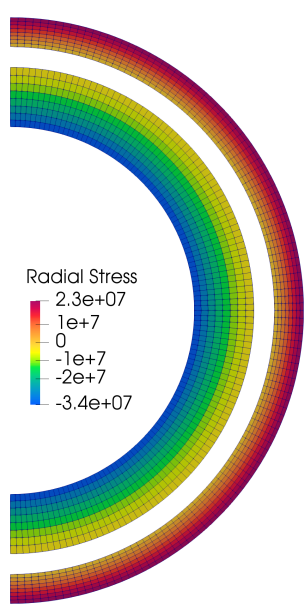

(d) $t=48313150 \mathrm{~s}$

Fig. 17. Radial stress during debonding of the buffer from the IPyC with a bond strength of $80 \mathrm{MPa}$ at $t=$ (a) $612481 \mathrm{~s}$, (b) $612491 \mathrm{~s}$, (c) $612502 \mathrm{~s}$, and (d) $48313150 \mathrm{~s}$.

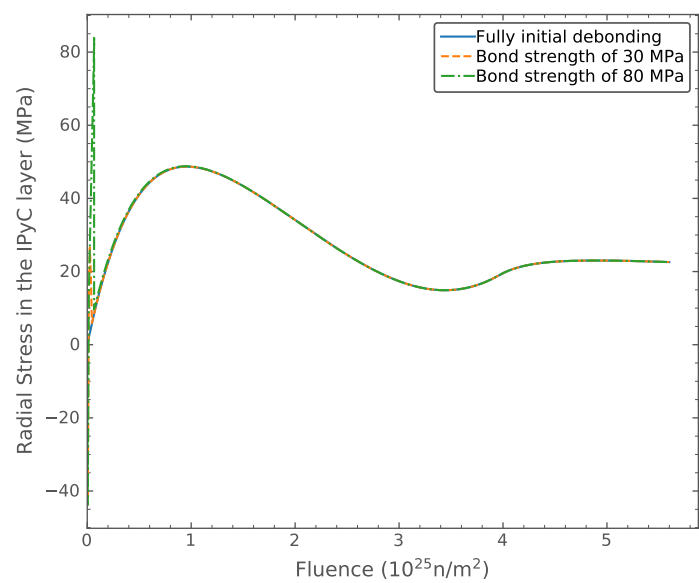

(a) Radial stress

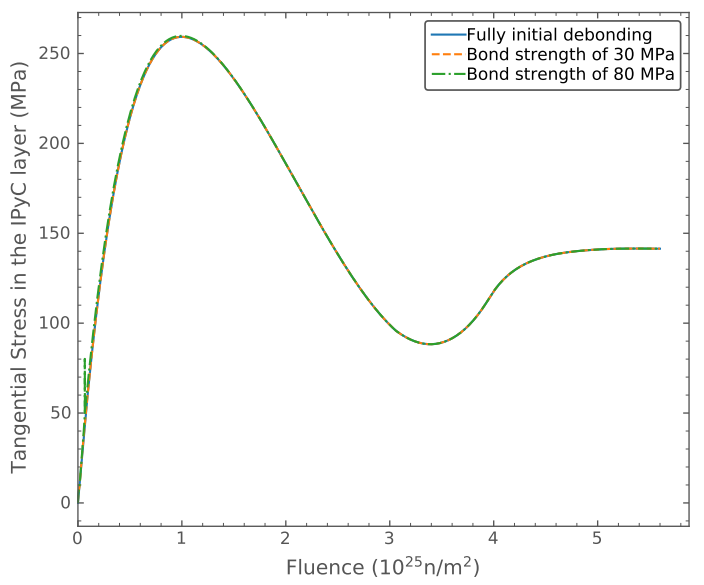

(b) Tangential stress

Fig. 18. Time history of stress in the IPyC layer during debonding of the buffer from the IPyC.

example was considered here to elucidate the effect of partial debonding on the IPyC fracture. As shown in Figure 19, the CZM interface was applied to only part of the buffer-IPyC interface, while keeping the top part fully connected. The damage evolution and radial stress of partial debonding between the buffer and IPyC layers is shown in Figure 20 and Figure 21, respectively. A stress concentration occurs at the debonded tip. The radial and tangential stresses in the IPyC layer are significantly higher than those produced by the fully debonded buffer and IPyC layers, and could potentially result in cracking of the IPyC layer. This is in good agreement with the experimental observation. 


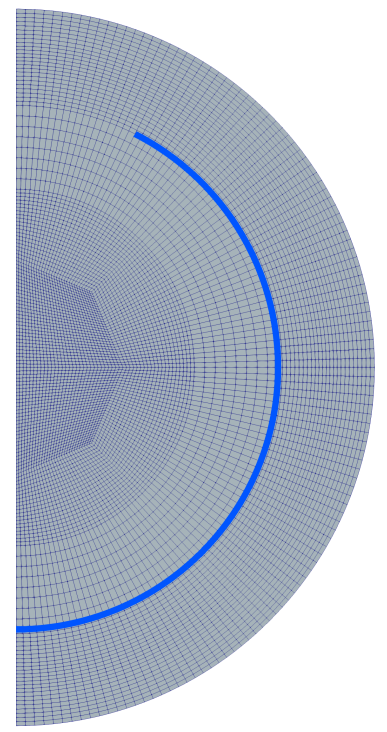

Fig. 19. Partial debonding of the buffer from the IPyC layer. The CZM interface is indicated in blue.

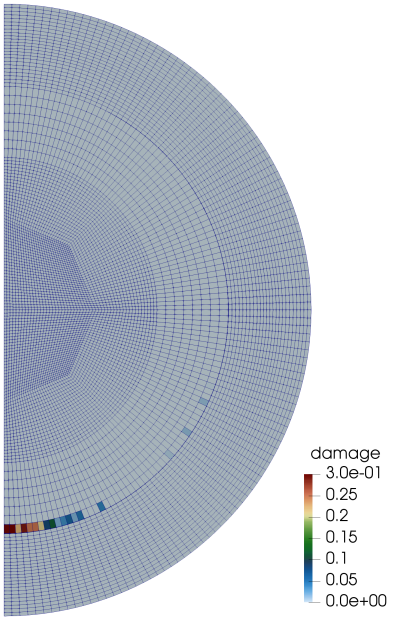

(a) $t=1151191 \mathrm{~s}$

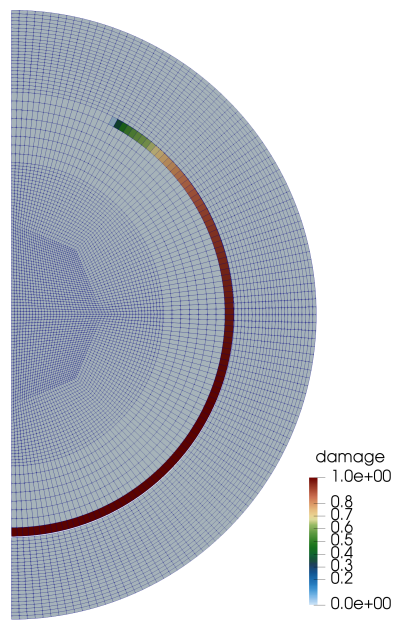

(b) $t=1151363 \mathrm{~s}$

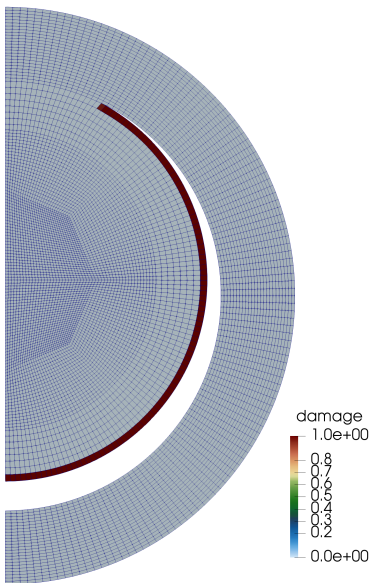

(c) $t=48313150 \mathrm{~s}$

Fig. 20. Damage evolution during partial debonding of the buffer from the IPyC at $t=$ (a) $1151191 \mathrm{~s}$, (b) $1151363 \mathrm{~s}$, and (c) $48313150 \mathrm{~s}$.

\section{3) Debonding of the buffer from the IPyC and IPyC cracking}

In addition to the debonding of the buffer from the IPyC layer, the cracking of the IPyC layer was also simulated using the CZM. The normal strength of the IPyC cracking was set at $80 \mathrm{MPa}$. From Figure 23 and Figure 24, cracking occurs after the buffer and IPyC were fully debonded. Such a crack leads to high local tensile stress in the $\mathrm{SiC}$ layer adjacent to the cracked IPyC, potentially causing failure of the SiC layer. The time history of stress in the IPyC and SiC layers is shown in Figure 25. The debonding does not have a large impact on the stress in the 


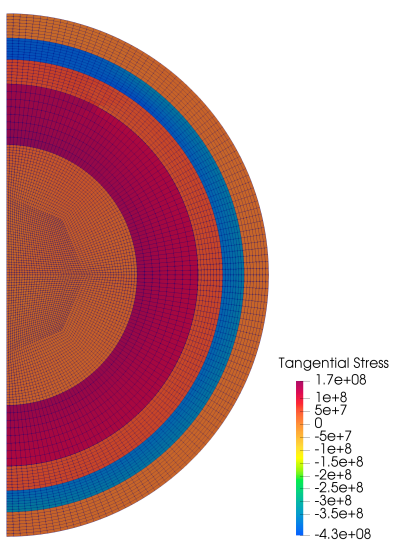

(a) $t=1151191 \mathrm{~s}$

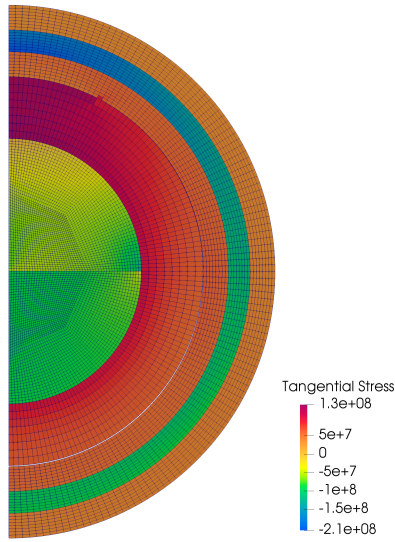

(b) $t=1151363 \mathrm{~s}$

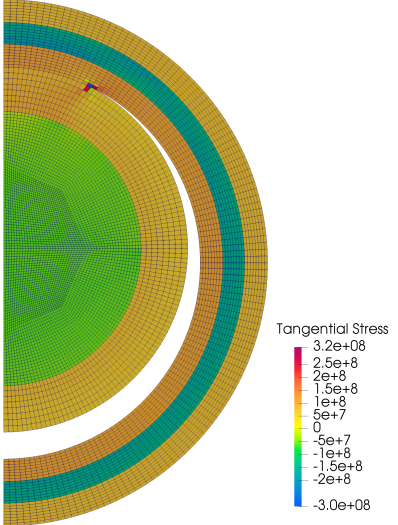

(c) $t=48313150 \mathrm{~s}$

Fig. 21. Tangential stress during partial debonding of the buffer from the IPyC at $t=$ (a) $1151191 \mathrm{~s}$, (b) $1151363 \mathrm{~s}$, and (c) $48313150 \mathrm{~s}$.

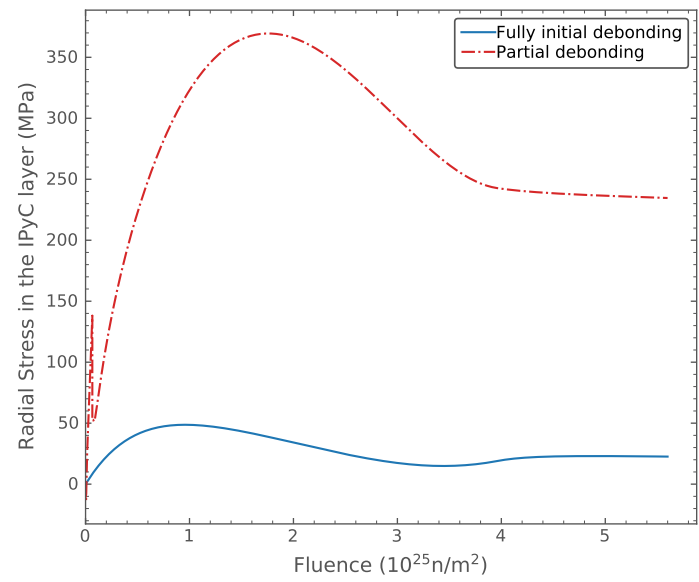

(a) Radial stress

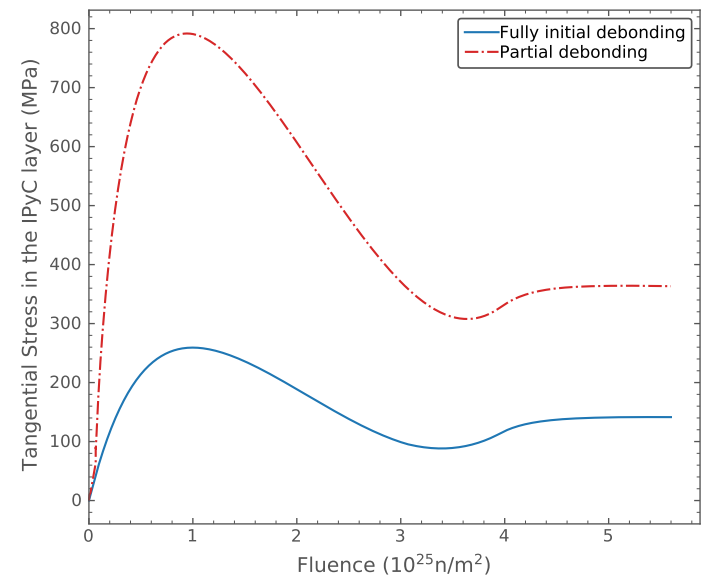

(b) Tangential stress

Fig. 22. Time history of stress in the IPyC layer during partial debonding of the buffer from the IPyC.

IPyC layer, whereas the cracking causes the $\mathrm{SiC}$ stress to change from compressive to tensile.

4) Debonding of the buffer, IPyC, and SiC layers

In the last example, debonding of both the buffer from the IPyC layer and the IPyC from the SiC layer was simulated. The damage evolution and radial stress is shown in Figure 26 and Figure 27, respectively. Although the bond strength of the buffer-IPyC and IPyC-SiC layers is the same, debonding of the buffer from the IPyC occurs first, because the radial stress between the buffer and IPyC layers reaches the critical bond strength sooner. The time history of tangential stress in the IPyC and SiC layers is shown in Figure 28. 


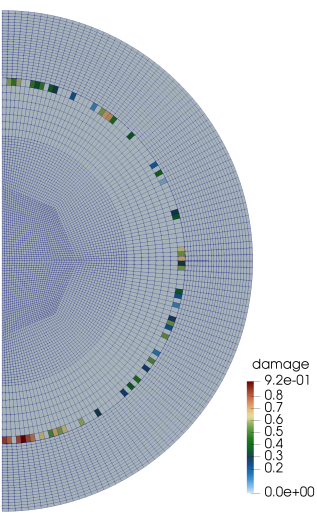

(a) $t=411919 \mathrm{~s}$

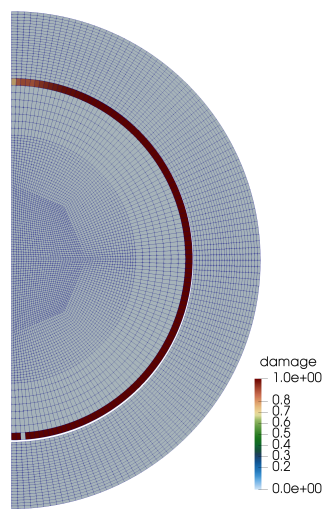

(b) $t=1016731 \mathrm{~s}$

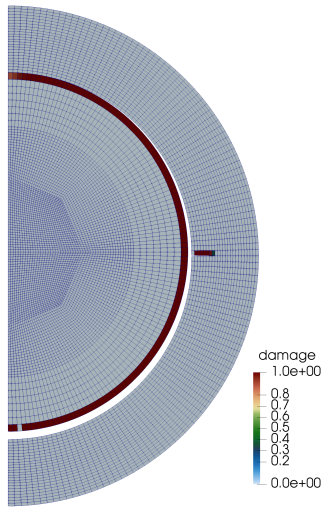

(c) $t=4127131 \mathrm{~s}$

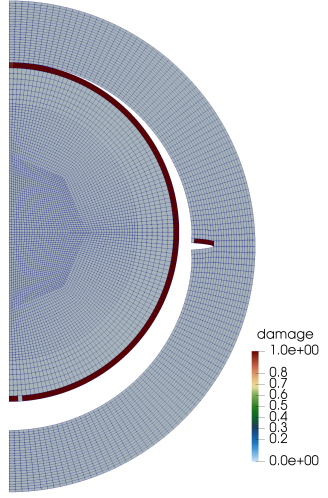

(d) $t=48313150 \mathrm{~s}$

Fig. 23. Damage evolution during debonding of the buffer from the IPyC and IPyC cracking at $t=$ (a) $411919 \mathrm{~s}$, (b) $1016731 \mathrm{~s}$, (c) $4127131 \mathrm{~s}$, and (d) $48313150 \mathrm{~s}$.

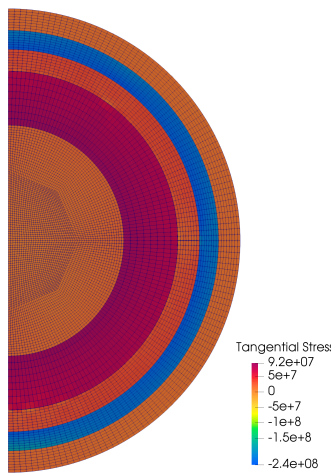

(a) $t=411919 \mathrm{~s}$

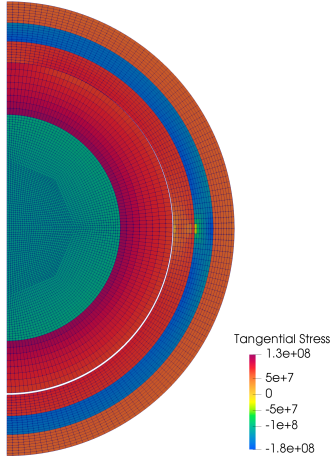

(b) $t=1016731 \mathrm{~s}$

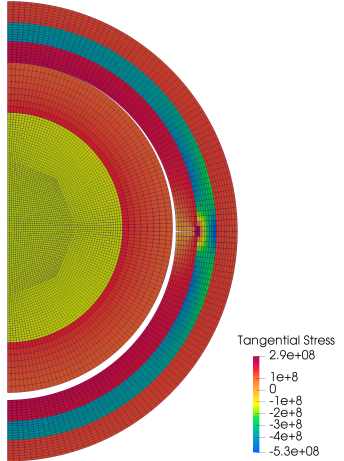

(c) $t=4127131 \mathrm{~s}$

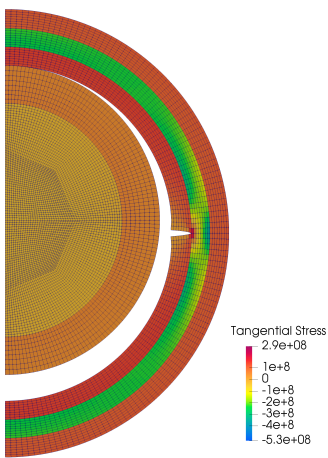

(d) $t=48313150 \mathrm{~s}$

Fig. 24. Tangential stress during debonding of the buffer from the IPyC and IPyC cracking at $t=$ (a) $411919 \mathrm{~s}$, (b) $1016731 \mathrm{~s}$, (c) $4127131 \mathrm{~s}$, and (d) $48313150 \mathrm{~s}$.

\section{E. Incorporate Debonding Failure into Monte Carlo Scheme}

\section{1) Stress correlation for IPyC cracking}

The maximum tangential stress histories in the SiC layer for both a debonded and an intact particle are shown in Figure 29. The maximum SiC stress in a debonded particle is approximated as:

$$
\sigma_{\text {IPyC-SiC-debonding }}=\frac{\bar{\sigma}_{2 \mathrm{D}}}{\bar{\sigma}_{1 \mathrm{D}}} \sigma_{1 \mathrm{D}}
$$

where $\bar{\sigma}_{2 \mathrm{D}}$ and $\bar{\sigma}_{1 \mathrm{D}}$ are the maximum stress calculated in the two-dimensional (2-D) and 1-D analyses, respectively, at the mean values for a specified batch of particles. Upon varying statistical parameters, the maximum stress in the $\mathrm{SiC}$ layer is determined from the 1-D finite element solution for $\sigma_{1 \mathrm{D}}$. The mean strength of the 2-D model, as evaluated at the maximum tangential stress state, will be used for 1-D analysis. 


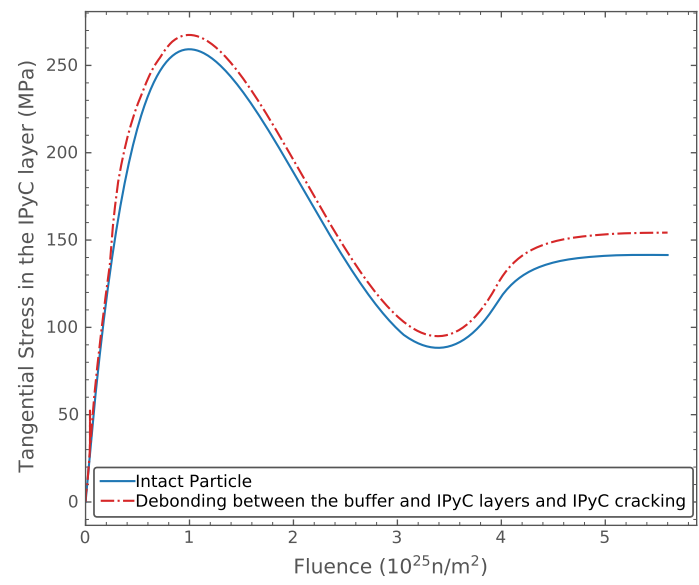

(a) Tangential stress in the IPyC layer

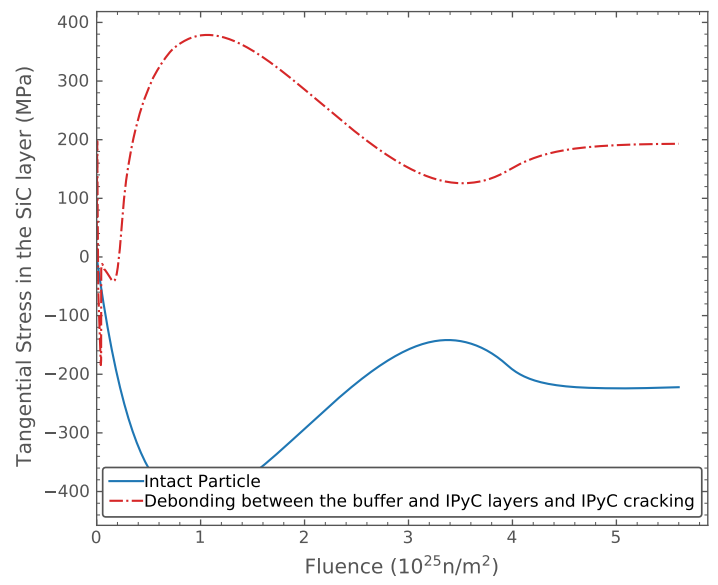

(b) Tangential stress in the $\mathrm{SiC}$ layer

Fig. 25. Time history of stress during debonding of the buffer from the IPyC and IPyC cracking.

\section{2) Monte Carlo Scheme}

Figure 30 depicts the methodology used to calculate the failure probability of a population of TRISO particles. This methodology relies on an MC scheme in which each particle analyzed is a realization of a set of statistically sampled parameters from the distributions of as-fabricated fuel characteristics (e.g., dimensions and densities) that can be found among the particles in a fuel element. For each sample, Bison runs a 1-D model of a TRISO particle over the irradiation history. For particles with localized flaws (i.e., aspherical particles, particles with cracked IPyC, and particles with debonded $\mathrm{IPyC}$ and $\mathrm{SiC}$ ), an adjustment of the maximum stress and effective mean strength will be made, as described in [4].

At each time step, the following failure mechanisms are checked:

- Pressure vessel failure of a spherical or aspherical particle: Failure occurs when the maximum tangential tensile stress in the $\mathrm{SiC}$ layer due to internal gas pressure exceeds the strength of the $\mathrm{SiC}$.

- $\mathrm{SiC}$ failure due to IPyC cracking: Cracking of the IPyC occurs when the maximum tangential tensile stress in the IPyC layer exceeds its strength. A cracked IPyC changes stress from compressive to tensile in the $\mathrm{SiC}$ layer. SiC failure occurs when the maximum tangential tensile stress of the $\mathrm{SiC}$ layer due to IPyC cracking exceeds its strength.

- $\mathrm{SiC}$ failure due to debonding between the IPyC and SiC layers: Debonding of the IPyC and SiC layers occurs when the maximum radial stress at the interface exceeds its bond strength. A stress concentration in the $\mathrm{SiC}$ layer occurs at the tip of the debonded region. $\mathrm{SiC}$ failure occurs when the maximum tangential tensile stress in the $\mathrm{SiC}$ layer exceeds its strength, due to debonding". 


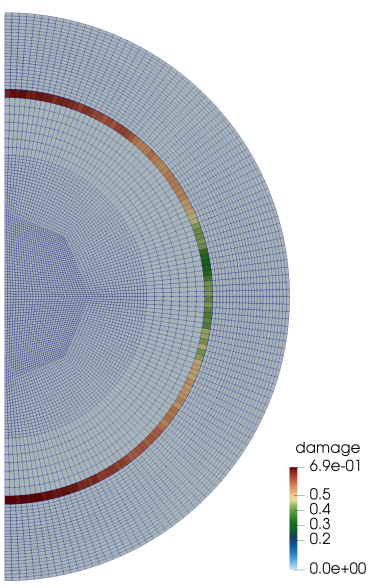

(a) $t=259200 \mathrm{~s}$

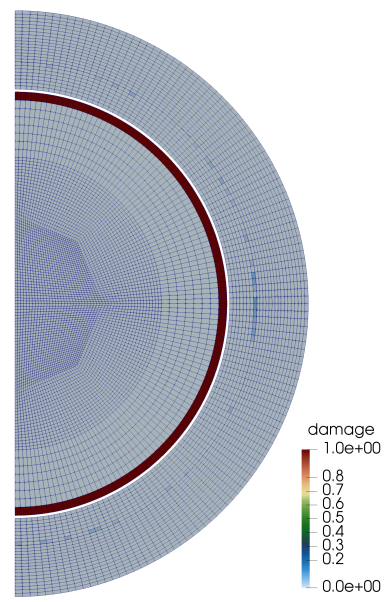

(b) $t=2548800 \mathrm{~s}$

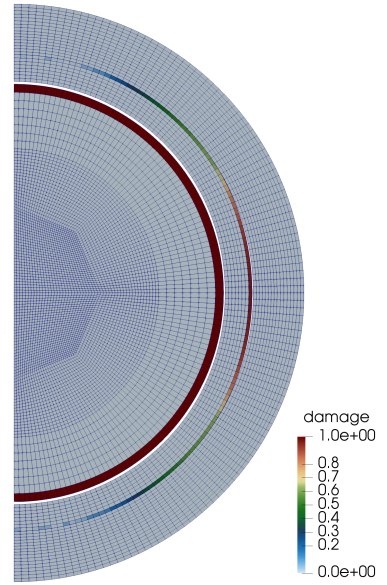

(c) $t=2582265 \mathrm{~s}$

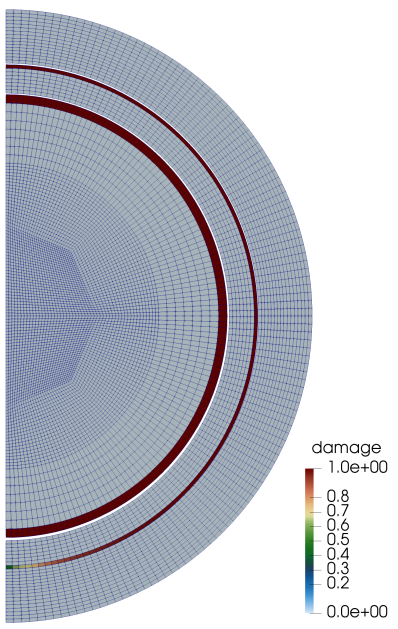

(d) $t=2582444 \mathrm{~s}$

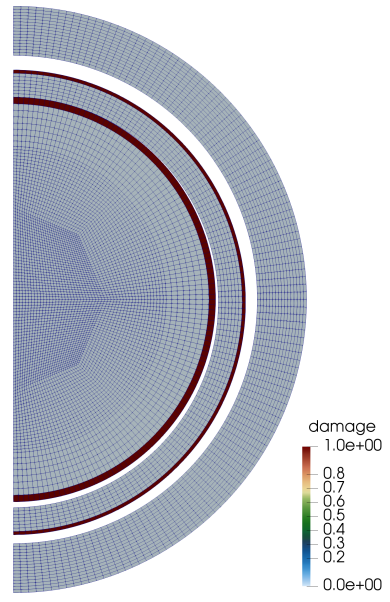

(e) $t=48313150 \mathrm{~s}$

Fig. 26. Damage evolution during debonding of the buffer from the IPyC and the IPyC from the SiC layer at $t=$ (a) $259200 \mathrm{~s}$, (b) $2548800 \mathrm{~s}$, (c) $2582265 \mathrm{~s}$, (d) $2582444 \mathrm{~s}$, and (e) $48313150 \mathrm{~s}$. 


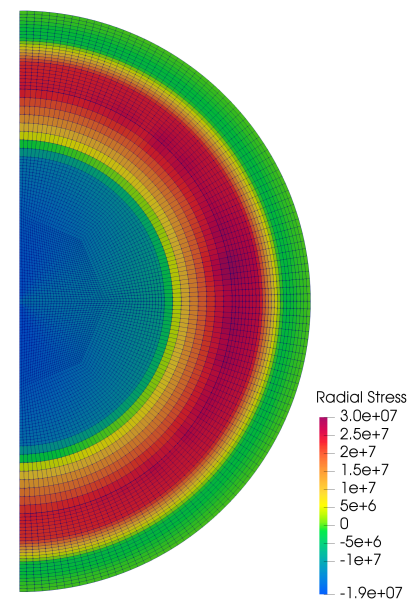

(a) $t=259200 \mathrm{~s}$

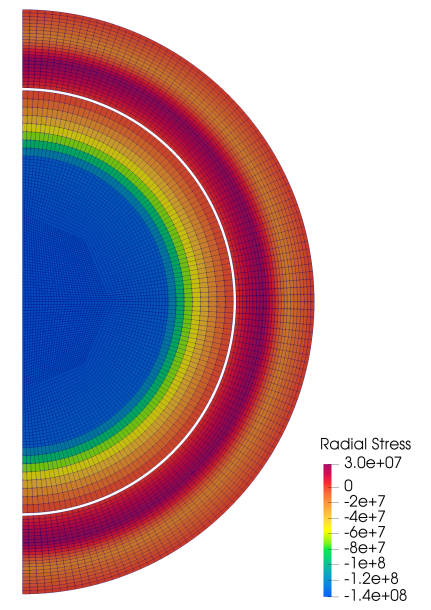

(b) $t=2548800 \mathrm{~s}$

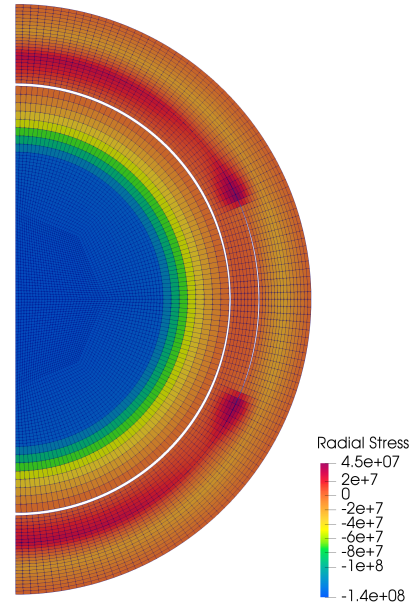

(c) $t=2582265 \mathrm{~s}$

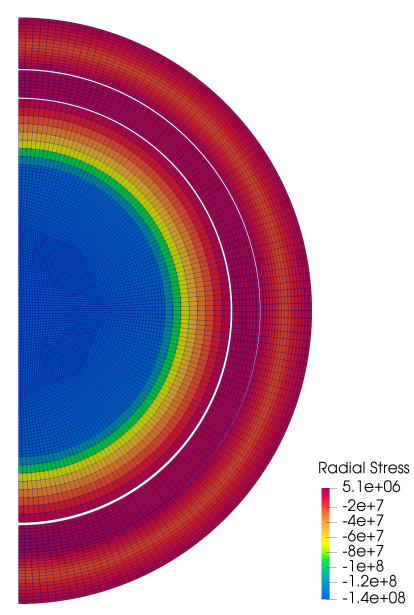

(d) $t=2582444 \mathrm{~s}$

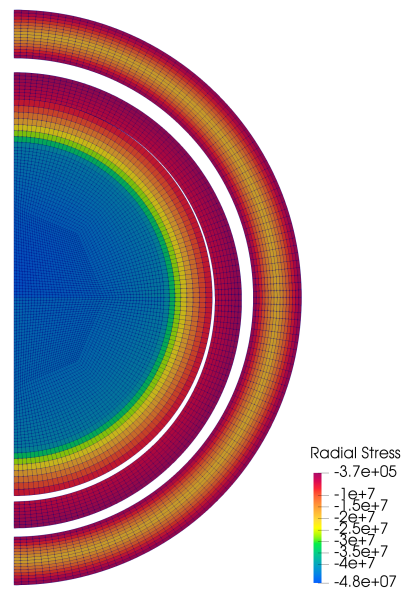

(e) $t=48313150 \mathrm{~s}$

Fig. 27. Radial stress during debonding of the buffer from the IPyC and the IPyC from the SiC layer at $t=$ (a) $259200 \mathrm{~s}$, (b) $2548800 \mathrm{~s}$, (c) $2582265 \mathrm{~s}$, (d) $2582444 \mathrm{~s}$, and (e) $48313150 \mathrm{~s}$. 


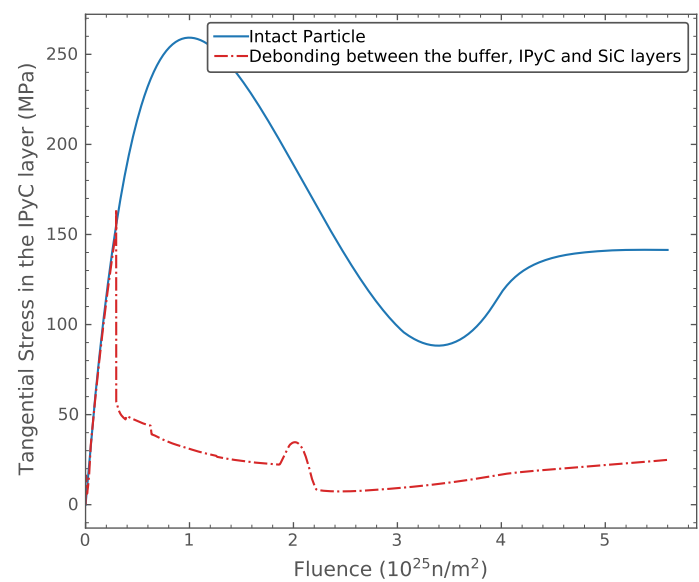

(a) Tangential stress in the IPyC layer

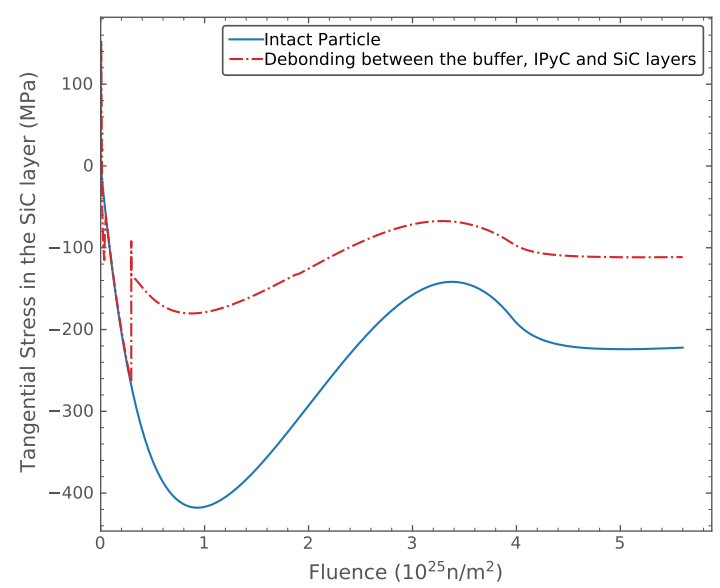

(b) Tangential stress in the $\mathrm{SiC}$ layer

Fig. 28. Time history of stress during debonding of the buffer, IPyC, and SiC layers.

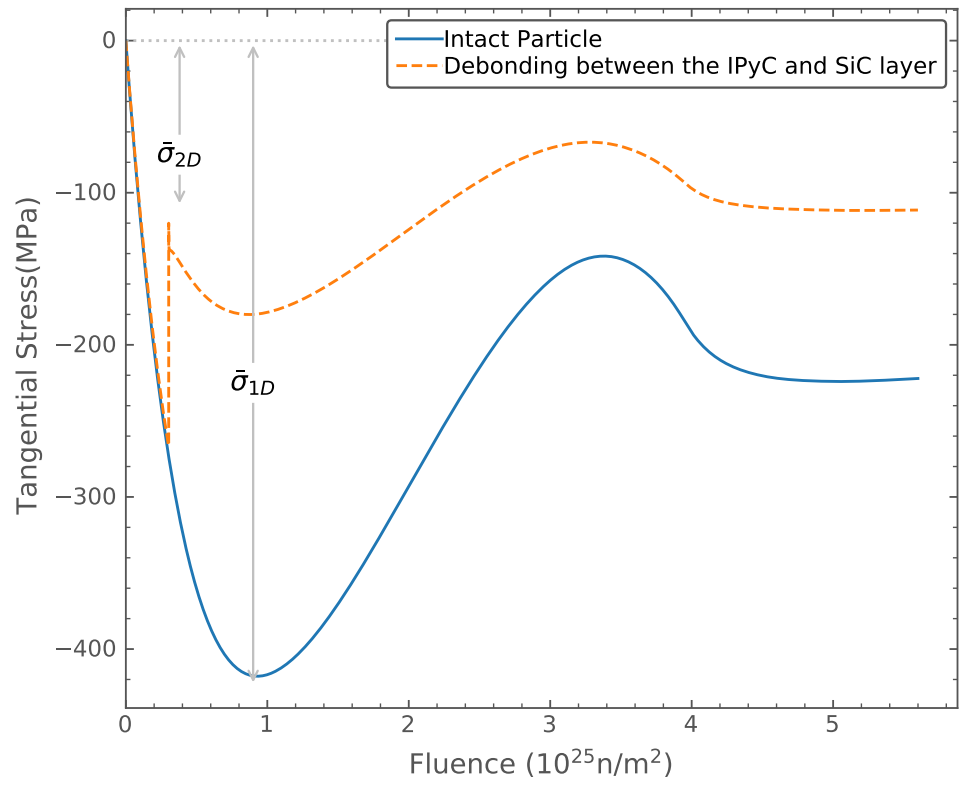

Fig. 29. Time histories of maximum tangential stress in the SiC layer for a 2-D model of a particle with debonding between the IPyC and SiC layer, as compared with a 1-D model of an intact particle. These are used to develop the stress correlation for debonding. 




Fig. 30. MC scheme employed by Bison for calculating the failure probability of TRISO particles. 


\section{EFFICIENT HIgh-FIDELITY TRISO STATISTICAL FAILURE ANALYSIS}

The ability of the TRISO fuel to contain fission products is largely dictated by the quality of the manufacturing process, since most of the fission product release is expected to occur due to coating layer failure in a small number of particles at the defect locations. To account for statistical variation in physical dimensions and material properties from one particle to another, an MC scheme was developed in fiscal year 2020 [4] to compute the failure probability for a statistically sampled batch of particles. One limitation of the MC scheme is that the number of samples must be very large to compute a relatively low failure probability, and the computational resources needed for the task are tremendous. Therefore, it is important to implement a new fast approach in Bison to greatly enhance the efficiency of the failure probability calculation.

\section{A. Failure Modes}

Several potential failure mechanisms for TRISO fuel, outlined in the sections below, are considered in the current work. Other failure modes besides those discussed herein are both possible and potentially important, and techniques for considering them are in active development. Several changes made on TRISO material models, as described in Section II, are utilized to increase the accuracy of the simulation results.

1) Pressure vessel failure

Early on during irradiation, PyC shrink, compressing the $\mathrm{SiC}$ layer. As irradiation progresses, the creep of the PyC layers tends to relax some of this compressive stress. In addition, the buildup of fission gas pressure tends to put all the coating layers under tension. Since the CO production in a UCO kernel is relatively small, the gas pressure is usually too low to cause the tangential stress in the $\mathrm{SiC}$ layer to become tensile. Therefore, pressure vessel failure is unlikely to occur for an intact TRISO UCO kernel at low and intermediate temperatures.

2) Irradiation-induced IPyC failure leading to SiC cracking

During irradiation, shrinkage of the PyC layers causes significant tensile stress in those layers. If the stress exceeds the tensile strength of the material, a radial crack can form in a PyC layer. Such a crack leads to high local tensile stress in the $\mathrm{SiC}$ layer adjacent to that cracked PyC layer, potentially causing failure of the SiC layer and, therefore, of the particle. In Figure 31a, a discrete crack in the IPyC layer is represented in the finite element model. The plenum pressure caused by fission gases is applied on crack surfaces. As shown in Figure $31 \mathrm{~b}$, the stress in the $\mathrm{SiC}$ layer near the crack tip is significantly elevated due to the development of cracking in the IPyC layer. The pressure on crack surfaces makes a larger difference in the $\mathrm{SiC}$ stress when there is sufficient buildup of fission gas pressure near the end of the irradiation.

3) Pressure vessel failure of an aspherical particle

A single flat facet on one side of the particle created during fabrication is a common cause of aspherical behavior in particle fuel. During irradiation, the faceted portion of the particle acts as a flat plate that retains the internal gas pressure. If the pressure builds up high enough, this results in a local region of tensile stress in the central portion of the plate, potentially contributing to particle failure. As shown in Figure 32b, the use of local normal vectors predicts a much higher tensile stress at the end of the irradiation. This could have a significant impact on the failure probability calculation.

\section{B. Weibull Failure Theory}

A Weibull failure criterion is used to determine vessel failure for the IPyC layer and SiC layer. The maximum stress, $\sigma_{c}$, is compared to a strength sampled from a Weibull distribution featuring mean strength $\sigma_{m s}$ and Weibull modulus $m$. Failure occurs when $\sigma_{c}$ exceeds the sampled strength. The cumulative distribution function for the Weibull distribution is given as:

$$
P=1-\exp \left(-\left[\frac{\sigma_{c}}{\sigma_{m s}}\right]^{m}\right)
$$




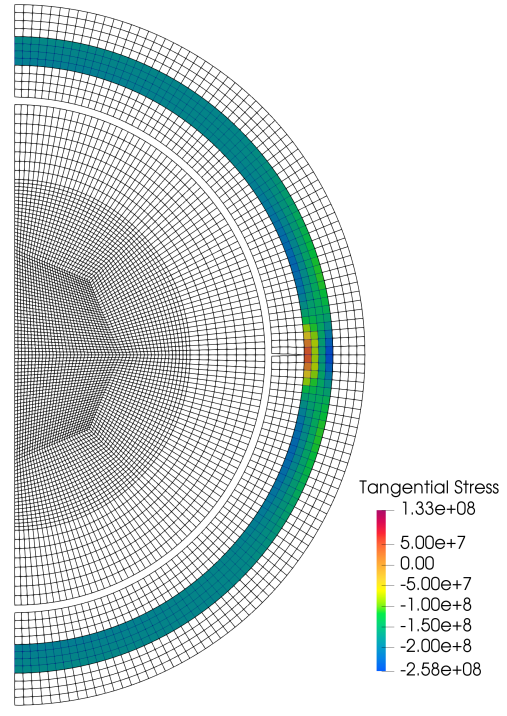

(a) Stress contour $(\mathrm{Pa})$ for $\mathrm{T}=1300^{\circ} \mathrm{C}$ when maximum tangential stress is reached. For clarity, only stresses in the SiC layer are shown.

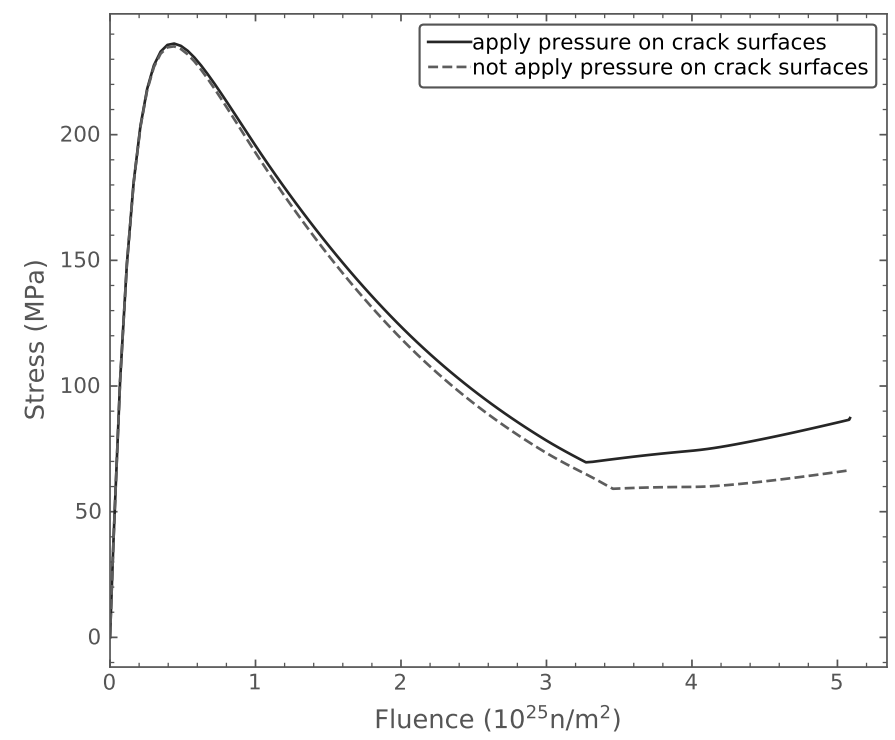

(b) Time history of peak inner-wall tangential stress in the SiC layer.

Fig. 31. 2-D axisymmetric model of postulated cracking of the IPyC layer, and comparison between applying and not applying pressure on crack surfaces.

The effective mean strength $\sigma_{m s}$ is given as:

$$
\sigma_{m s}=\frac{\sigma_{0}}{I_{n}^{1 / m}}
$$

where $\sigma_{0}$ is the characteristic strength. The integral, $I_{n}$, is a normalized integration of the stress distribution using the principle of independent action (PIA) model as follows:

$$
I_{n}=\frac{\int_{V}\left(\sigma_{1}^{m}+\sigma_{2}^{m}+\sigma_{3}^{m}\right) d V}{\sigma_{c}^{m}}
$$

where $\sigma_{c}$ is the maximum value calculated for a principal stress anywhere in the volume and $\sigma_{1}, \sigma_{2}$, and $\sigma_{3}$ are three principal stresses. Negative principal stresses are not included in this integral because the compressive stresses do not contribute to fracture.

The Weibull modulus $(m)$ and characteristic strength $\left(\sigma_{0}\right)$ for the $\mathrm{SiC}$ are held constant throughout irradiation and are given as:

$$
m=6
$$

and

$$
\sigma_{0}=9.64 \times 10^{6} \mathrm{~Pa}-\mathrm{m}^{3 / 6}
$$

The Weibull modulus for the PyC is assumed to be 9.5 , which corresponds to a density of $1.9 \mathrm{~g} / \mathrm{cm}^{3}$.

The Weibull characteristic strength of the PyC $[9,10]$ is a function of anisotropy, and is determined from the 


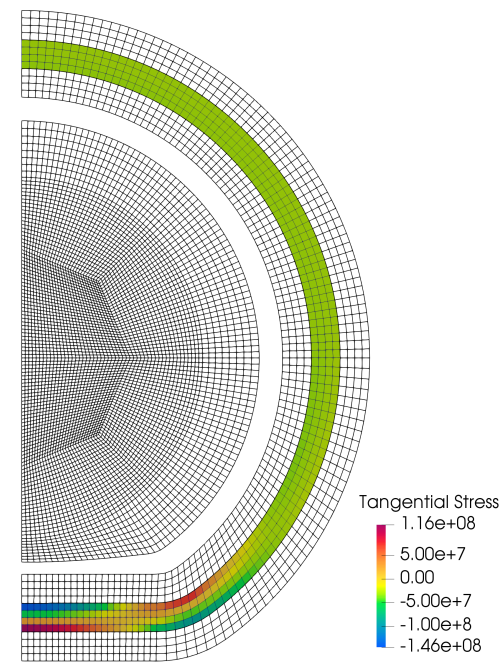

(a) Stress contour $(\mathrm{Pa})$ for $\mathrm{T}=1300^{\circ} \mathrm{C}$ at end of irradiation, with an aspect ratio of 1.04 . For clarity, only stresses in the $\mathrm{SiC}$ layer are shown.

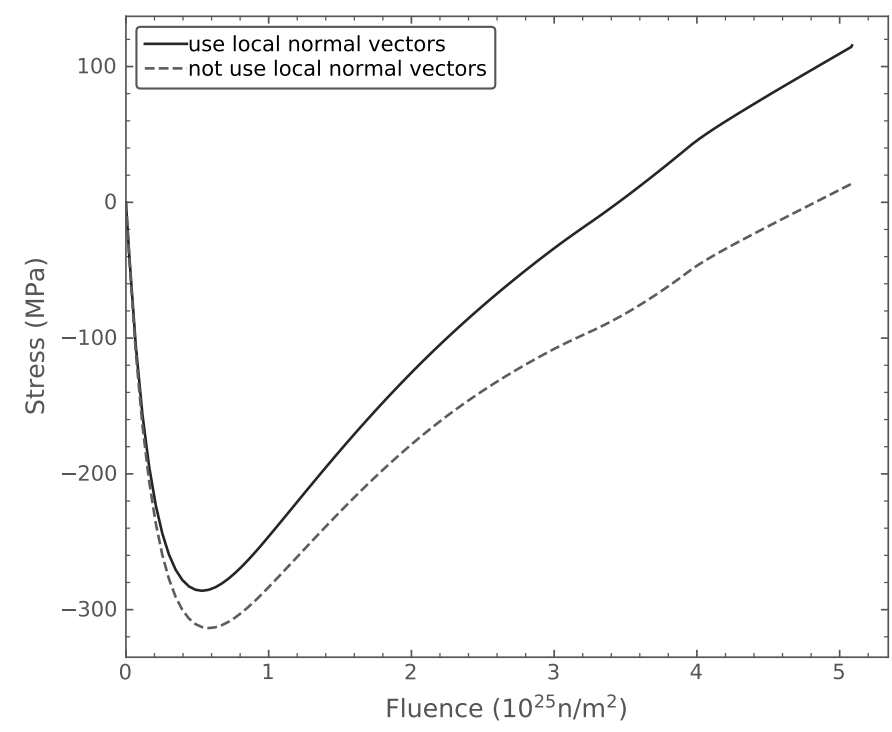

(b) Time history of stress in the faceted portion of the $\mathrm{SiC}$ layer. Results obtained with and without using the local normal vectors are shown for comparison.

Fig. 32. 2-D axisymmetric model of an aspherical particle, and time-dependent results for that model.

following equation for room temperature:

$$
\sigma_{0}=10^{6}\left(154.46 X^{2}-141.1 X\right)
$$

where $X$ is a fitting parameter with a default value of 1.02 . The characteristic strength has units of Pa- $\mathrm{m}^{3 / 9.5}$ when the Weibull modulus is 9.5 .

To account for other temperatures and non-zero fluence, Equation 23 is multiplied by the following factor:

$$
\text { factor }=[(1+0.23 \phi)(1+0.00015 T)]^{1 / 2}
$$

where: $\phi=$ the fast neutron fluence $\left(10^{25} \mathrm{n} / \mathrm{m}^{2}, E>0.18 \mathrm{MeV}\right)$

$T=$ the temperature $\left({ }^{\circ} \mathrm{C}\right)$.

\section{Higher Order Stress Concentrations}

For computational efficiency, it is helpful if each sample is evaluated using a 1-D model. However, stress concentrations due to the presence of phenomena such as cracking must be characterized using a higher dimensional model. To account for these multi-dimensional phenomena within a 1-D TRISO model, a high-dimensional failure simulation is performed to obtain the mean effective strength and stress correlation function, based on a multidimensional stress distribution. Those values are used in the 1-D model to make adjustments to the stress in failure determination.

Bison considers a statistical variation of the IPyC, SiC, and OPyC layer thickness because they strongly affect particle failure. To obtain each correlation function $h(\Delta v)$, we typically sample seven points where $\Delta v=-3 \mathrm{~s},-2 \mathrm{~s}$, $-\mathrm{s}, 0, \mathrm{~s}, 1 \mathrm{~s}, 2 \mathrm{~s}$, and $3 \mathrm{~s}$, and $s$ is the standard deviation of the thickness. At the midpoint, $h(\Delta v)$ has a value of 1 
because $\Delta v=0$. At other data points, we perform both multi- and one-dimension analysis to determine the value of the correlation function. A polynomial curve fit is finally performed on the data points to generate a quadratic $h(\Delta v)$ function. The correlation functions for the IPyC cracking and aspherical particle are outlined below.

1) Stress correlation for IPyC cracking

The maximum $\mathrm{SiC}$ stress in a cracked particle is approximated as:

$$
\sigma_{\text {ipyc-cracking }}=\frac{\bar{\sigma}_{2-\mathrm{D}}}{\bar{\sigma}_{1-\mathrm{D}}} \sigma_{1-\mathrm{D}}\left(v_{\mathrm{IPyC}}, v_{\mathrm{SiC}}, v_{\mathrm{OPyC}}\right) h_{\mathrm{IPyC}}\left(\Delta v_{\mathrm{IPyC}}\right) h_{\mathrm{SiC}}\left(\Delta v_{\mathrm{SiC}}\right) h_{\mathrm{OPyC}}\left(\Delta v_{\mathrm{OPyC}}\right)
$$

where: $\quad \bar{\sigma}_{2-\mathrm{D}}=$ the max. stress calculated in 2-D analyses at the mean values for a specified batch of particles $\bar{\sigma}_{1-\mathrm{D}}=$ the max. stress calculated in 1-D analyses at the mean values for a specified batch of particles.

Upon varying statistical parameters, the maximum stress in the $\mathrm{SiC}$ layer is determined from the 1-D finite element solution for $\sigma_{1-\mathrm{D}}$ and the correlation functions. An example stress correlation function for IPyC cracking is shown in Figure 33. The mean strength of the 2-D model, as evaluated at the maximum tangential stress state, will be used for 1-D analysis.

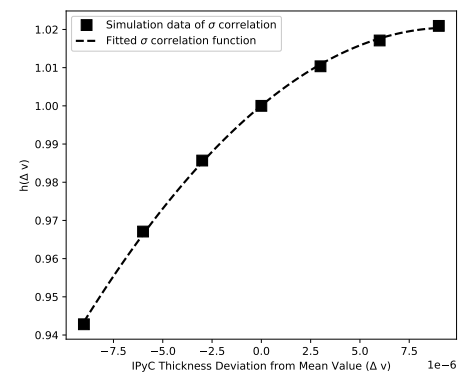

(a) Stress correlation function of IPyC layer thickness for IPyC cracking.

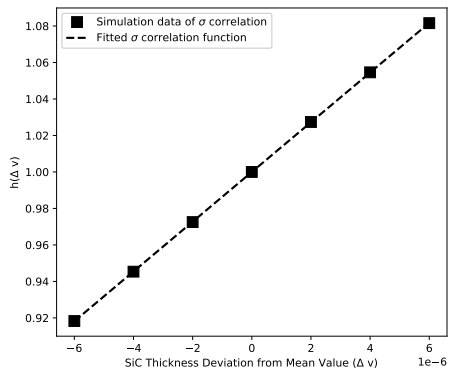

(b) Stress correlation function of $\mathrm{SiC}$ layer thickness for IPyC cracking.

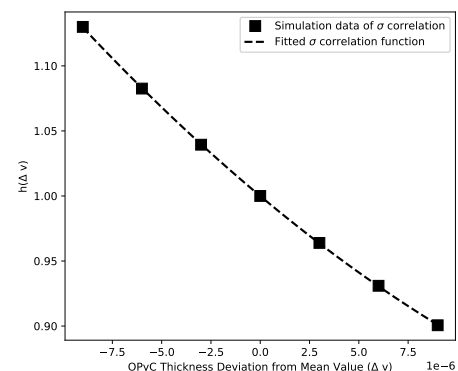

(c) Stress correlation function of $\mathrm{OPyC}$ layer thickness for IPyC cracking.

Fig. 33. Example of stress correlation functions for IPyC cracking.

\section{2) Stress correlation for an aspherical particle}

The tangential stress histories for representative faceted and spherical fuel particles are shown in Figure 34. In evaluating the effect of asphericity, a second term is added to correctly estimate the maximum stress, $\sigma_{c}$, for an aspherical particle:

$$
\begin{aligned}
\sigma_{\text {aspherical-particle }} & =\frac{\bar{\sigma}_{2-\mathrm{D}}}{\bar{\sigma}_{1-\mathrm{D}}} \sigma_{1-\mathrm{D}-\mathrm{min}}\left(v_{\mathrm{IPyC}}, v_{\mathrm{SiC}}, v_{\mathrm{OPyC}}\right) h_{\mathrm{IPyC}}\left(\Delta v_{\mathrm{IPyC}}\right) h_{\mathrm{SiC}}\left(\Delta v_{\mathrm{SiC}}\right) h_{\mathrm{OPyC}}\left(\Delta v_{\mathrm{OPyC}}\right) \\
& +\frac{\Delta \bar{\sigma}_{2-\mathrm{D}}}{\Delta \bar{\sigma}_{1-\mathrm{D}}} \Delta \sigma_{1-\mathrm{D}}\left(v_{\mathrm{IPyC}}, v_{\mathrm{SiC}}, v_{\mathrm{OPyC}}\right) h_{\mathrm{IPyC}-\Delta}\left(\Delta v_{\mathrm{IPyC}}\right) h_{\mathrm{SiC}-\Delta}\left(\Delta v_{\mathrm{SiC}}\right) h_{\mathrm{OPyC}-\Delta}\left(\Delta v_{\mathrm{OPyC}}\right)
\end{aligned}
$$

where $\Delta \bar{\sigma}_{2-\mathrm{D}}, \Delta \bar{\sigma}_{1-\mathrm{D}}$, and $\Delta \sigma_{1-\mathrm{D}-\mathrm{min}}$ are changes in the stresses $\bar{\sigma}_{2-\mathrm{D}}, \bar{\sigma}_{1-\mathrm{D}}$, and $\sigma_{1-\mathrm{D}-\mathrm{min}}$, respectively, going from the minimum to the end of irradiation. If a second extremum (or maximum) occurs before the end of irradiation, $\Delta \bar{\sigma}_{2-\mathrm{D}}, \Delta \bar{\sigma}_{1-\mathrm{D}}$, and $\Delta \sigma_{1-\mathrm{D}}$ are taken as changes in these stresses, going from minimum to maximum. The additional term is needed because pressure vessel failure of aspherical particles typically occurs after the first minimum stress is reached, when shrinkage effects from the $\mathrm{PyC}$ are decreasing and the inner pressure is continuing to accumulate. An example stress correlation function for aspherical particles is shown in Figure 34. The mean effective strength of the 2-D model evaluated at the end of irradiation will be used in 1-D analysis. 


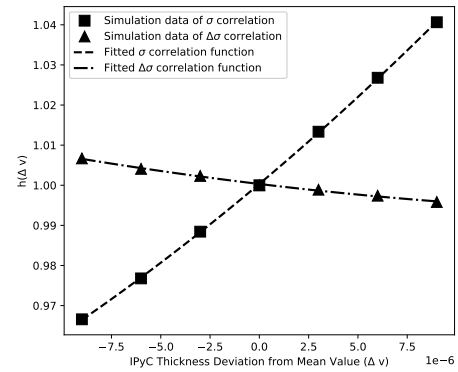

(a) Stress correlation function of IPyC layer thickness for an aspherical particle.



(b) Stress correlation function of $\mathrm{SiC}$ layer thickness for an aspherical particle.



(c) Stress correlation function of OPyC layer thickness for an aspherical particle.

Fig. 34. Example of stress correlation functions for an aspherical particle.

\section{Direct Integration Scheme}

1) Integration of failure probability function

In an integration approach, the failure probability (Equation 19) for a particle batch will be formulated in terms of an integral. The integral will involve Gaussian statistical distributions for particle design parameters. The failure probability integral is given as:

$$
P=\frac{1}{\sqrt{2 \pi}^{n} D_{i} D_{j} \ldots} \int_{-\infty}^{+\infty} \exp \left(\frac{-\left[v_{i}-\mu_{i}\right]^{2}}{2-\mathrm{D}_{i}^{2}}\right) \int_{-\infty}^{+\infty} \exp \left(\frac{-\left[v_{j}-\mu_{j}\right]^{2}}{2-\mathrm{D}_{j}^{2}}\right) \ldots\left\{1-\exp \left(-\left[\frac{\sigma_{c}\left(v_{i}, v_{j} \ldots\right)}{\sigma_{m s}}\right]^{m}\right)\right\}
$$

Numerically evaluating the integration requires an appropriate quadrature rule. A quadrature rule for the normal probability density function (PDF) $\phi(x)$ is a set of $N_{q}$ points $x_{q}$ and weights $w_{q}$ for which the integral can be estimated:

$$
\int_{-\infty}^{+\infty} f(x) \phi(x) d x \approx Q(f)=\sum_{q=1}^{N_{q}} w_{q} \cdot f\left(x_{q}\right)
$$

A quadrature rule with precision $k$ will exactly integrate all polynomials of degree $k$ or less. A Gauss-Hermite quadrature rule for the normal PDF will achieve a precision of $2 N_{q}-1$. In general, a multi-dimensional quadrature grid is a combination of 1-D quadrature rules. For example, a tensor grid can be described as:

$$
\mathcal{A}\left(D, N_{q}\right)=Q_{1}^{N_{q}} \otimes \cdots \otimes Q_{D}^{N_{q}}
$$

where $D$ is the number of dimensions and $Q_{d}^{j}$ is the one-dimensional quadrature set for dimension $d$ with $j$ points. Note that the number of points used by a tensor grid rule involves raising the number of points in the 1-D rule to the power of the dimensions. To overcome this limitation, a sparse grid method is proposed in order to provide accurate results at a much cheaper cost. The idea behind the efficiency of sparse grids is that, instead of taking the Cartesian product of full order quadrature rules, they use a combination of lower order quadrature rules to complete the monomial space. The sparse grid of a Gauss-Hermite quadrature can significantly reduce the number of points in a high-dimensional space. The sparse grid is described as: 


$$
\mathcal{A}\left(D, N_{q}\right)=\sum_{N_{q}-D \leq|i| \leq N_{q}-1}(-1)^{N_{q}+D-|i|-1}\left(\begin{array}{c}
N_{q}+D-1 \\
N_{q}+D-|i|-1
\end{array}\right)\left(Q_{1}^{i_{1}} \otimes \cdots \otimes Q_{D}^{i_{D}}\right)
$$

where $|i|=\sum_{d=1}^{D} i_{d}$. For more details on sparse grids, please refer to [19].

2) MOOSE Stochastic Tools module

Bison is built on the open-source MOOSE framework [20]. In addition to providing a computational framework for solving partial differential equations, MOOSE also supports stochastic analysis for MOOSE-based applications, including Bison, through its Stochastic Tools module. In previous work [4], the Stochastic Tools module was utilized to perform MC sampling. Because it uses MOOSE's built-in MultiApps system for memory-based data transfer and management of parallel computing resources, it is extremely efficient and scalable. In addition to MC simulation, the Stochastic Tools module can train and evaluate surrogate models using algorithms such as polynomial chaos, proper orthogonal decomposition (POD) reduced basis, and Gaussian processes. This work utilizes the quadrature-based sampling for normal distributions in order to integrate the failure probability function.

To verify implementation of the quadrature rule in MOOSE, we consider the following 2-D integral:

$$
I(f)=\frac{1}{\sqrt{2 \pi}} e^{-\frac{x^{2}}{2}} \int_{-\infty}^{+\infty} \frac{1}{\sqrt{2 \pi}} e^{-\frac{y^{2}}{2}} \int_{-\infty}^{+\infty} \frac{1}{x^{2}+y^{2}+1} d x d y
$$

MATLAB's function integral2() gives the (presumably) superior estimate of 0.4614554163 for the above integral. The integration values using MOOSE's tensor grid and sparse grid with different orders of the polynomials is shown in Figure 35. Both the tensor grid and sparse grid schemes converge to MATLAB's solution as the orders increase, though the convergence rate is faster for the tensor grid scheme. For $N_{q}=5$, the tensor grid and sparse grid require 36 and 55 quadrature points, and their locations are plotted in Figure 36a and Figure 36b, respectively. The number of quadrature points for different numbers of orders and dimensions for the tensor grid and sparse grid are provided in Table II. In statistical failure analysis of TRISO particles, there are typically 5-10 statistically varying parameters for which the sparse grid scheme can significantly reduce the number of quadrature points.

TABLE II. Number of quadrature points for tensor grid.

\begin{tabular}{clccc}
\hline $\mathbf{N}_{q}$ & Grid & $\mathbf{D = 3}$ & $\mathbf{D = 6}$ & $\mathbf{D = 9}$ \\
\hline \multirow{2}{*}{3} & Full & 64 & 4,096 & 262,144 \\
& Sparse & 28 & 91 & 190 \\
& Full & 216 & 46,656 & $10,077,696$ \\
5 & Sparse & 203 & 1,820 & 7,315 \\
& Full & 512 & 252,144 & $134,217,728$ \\
7 & Sparse & 840 & 18,563 & 134,596 \\
\hline
\end{tabular}

3) Methodology

A set of statistically varying parameters from the distributions of as-fabricated fuel characteristics (e.g., dimensions and densities) is considered in the integral of Equation 27 . The failure probability of IPyC cracking $\left(P_{\mathrm{IPyC}-\text { cracking }}\right)$, $\mathrm{SiC}$ failure due to IPyC cracking $\left(P_{\mathrm{SiC}-\mathrm{IPyC}-\text { cracking }}\right)$, and pressure vessel failure of aspherical particles $\left(P_{\mathrm{SiC}-\mathrm{PVF}}\right)$ is calculated using sparse grid integration. The overall $\mathrm{SiC}$ failure is given as:

$$
P_{\text {SiC-overall }}=P_{\text {IPyC-cracking }} \times P_{\text {SiC-IPyC-cracking }}+\left(1-P_{\text {IPyC-cracking }}\right) \times P_{\text {SiC-PVF }}
$$

As depicted in Figure 37 and Figure 38, two computation modes were developed to calculate failure probabilities depending on whether the 1-D or 2-D TRISO model is used for each sample. In the first mode, Bison runs the 


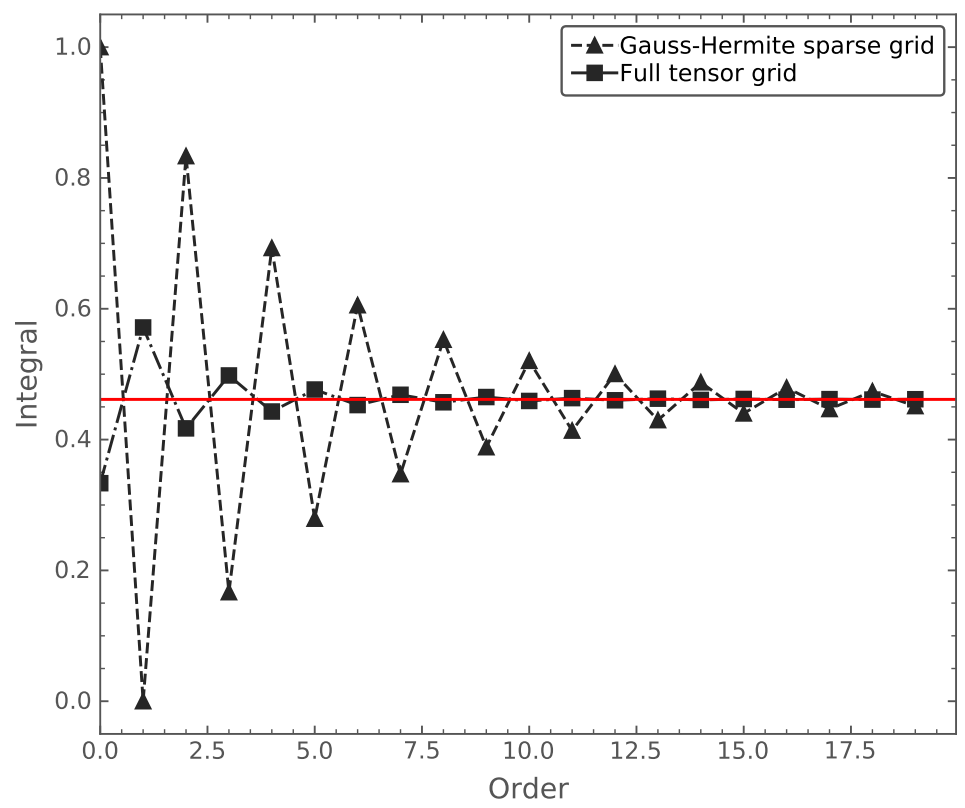

Fig. 35. Integration values using MOOSE's sparse grid with different orders of the polynomials.

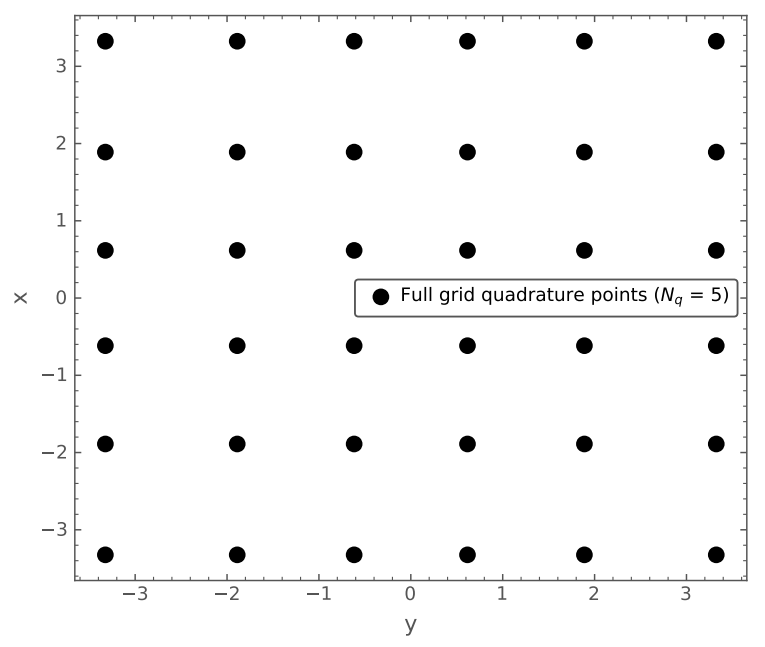

(a) Full Grid



(b) Sparse Grid

Fig. 36. Quadrature point location.

1-D model of a TRISO particle. For particles with localized flaws (i.e., aspherical particles and particles with cracked IPyC), an adjustment of the maximum stress and effective mean strength will be used, as described in Sections IV-C1 and IV-C2. In the second mode, Bison runs the 2-D model of a TRISO particle. A 2-D aspherical or spherical model will be used to determine the IPyC cracking failure probability and the PVF of the SiC layer, while a 2-D cracked IPyC model will be used to determine $\mathrm{SiC}$ layer failure due to IPyC cracking. Although the 1-D 
mode requires prior calculation of stress correlation factors and effective mean strength, its computation is much faster. On the other hand, the 2-D mode yields higher accuracy because it directly calculates failure probabilities for high-dimensional failure modes and does not require the use of any stress adjustment. The computation cost of the 2-D mode exceeds that of the 1-D mode, yet remains affordable due to the efficiency of the sparse grid scheme.
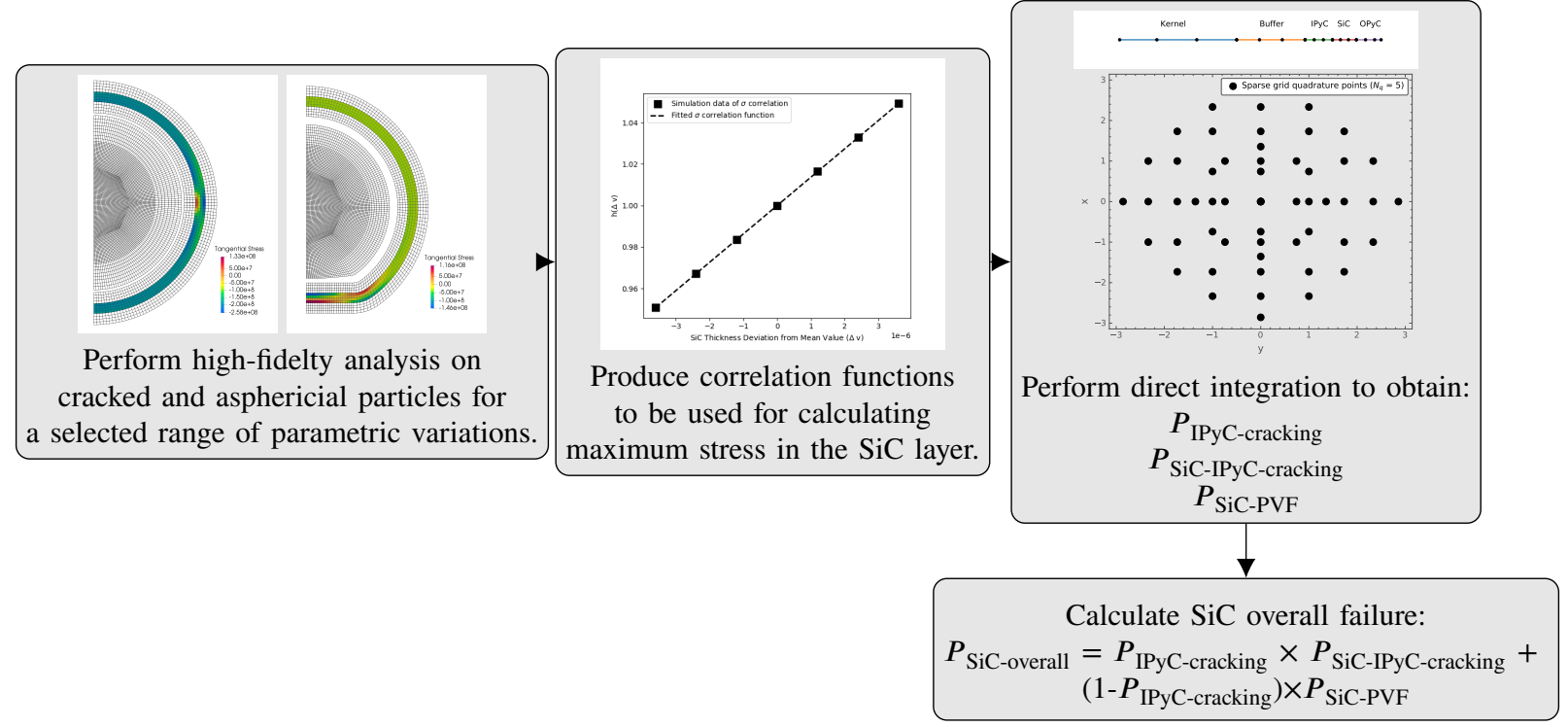

Fig. 37. Calculate the failure probability of TRISO particles in Bison: direct integration scheme using 1-D simulation with correlation functions.

\section{E. Benchmark Problems}

To demonstrate Bison's new capability for performing particle failure analysis on particle populations, benchmark testing was performed on a TRISO UCO fuel particle under representative irradiation conditions. These conditions are summarized in Table III. Temperatures of 700,1000 , and $1300^{\circ} \mathrm{C}$ were chosen so as to fall within the validity range of the material properties. The fuel parameters and their statistical distributions, which are indicated in the last column of Table IV, are based on the AGR-5/6/7 fuel specification.

TABLE III. Irradiation conditions for benchmark problems.

\begin{tabular}{lccc}
\hline & \multicolumn{3}{c}{ Conditions } \\
\cline { 2 - 4 } & 1 & 2 & 3 \\
\hline Effective full power days (EFPD) & 500 & 500 & 500 \\
Burnup (\%FIMA) & 13.5 & 13.5 & 13.5 \\
Fast fluence $\left(x 10^{25} \mathrm{n} / \mathrm{m}^{2}, E>0.18 \mathrm{MeV}\right)$ & 5.0 & 5.0 & 5.0 \\
Irradiation temperature $\left({ }^{\circ} \mathrm{C}\right)$ & 700 & 1000 & 1300 \\
\hline
\end{tabular}

The stress correlation functions and effective mean strength are pre-computed for each condition, and their values are listed in Table V and Table VI. The IPyC layer cracking using both MC and direct integration schemes are provided in Table VII. The number of samples for MC simulation is taken as $2000 / P_{\text {IPyC-cracking }}$. The results of $\mathrm{MC}$ and direct integration show reasonable agreement. For the direct integration scheme, different orders of $N_{q}$ are 




Fig. 38. Calculate the failure probability of TRISO particles in Bison: direct integration scheme using 2-D simulation.

TABLE IV. Fuel parameters in benchmark problems.

\begin{tabular}{llcc}
\hline \multirow{2}{*}{ Category } & Parameter & $\begin{array}{c}\text { Nominal values } \\
\pm \text { Standard } \\
\text { Deviation }\end{array}$ & $\begin{array}{c}\text { Statistical } \\
\text { Variation }\end{array}$ \\
\hline \multirow{5}{*}{ Fuel characteristics } & ${ }^{235} \mathrm{U}$ enrichment $(\mathrm{wt} \%)$ & 15.5 & \\
& Carbon/uranium (atomic ratio) & 0.4 & \\
& Oxygen/uranium (atomic ratio) & 1.5 & \\
\hline \multirow{5}{*}{ Particle geometry } & Kernel diameter $(\mu \mathrm{m})$ & $425 \pm 10$ & $\checkmark$ \\
& Buffer thickness $(\mu \mathrm{m})$ & $100 \pm 10$ & $\checkmark$ \\
& IPyC/OPyC thickness $(\mu \mathrm{m})$ & $40 \pm 3$ & $\checkmark$ \\
& SiC thickness $(\mu \mathrm{m})$ & $35 \pm 2$ & $\checkmark$ \\
& Particle asphericity $(\mathrm{SiC}$ aspect ratio) & 1.04 & $\checkmark$ \\
\hline \multirow{5}{*}{ Fuel properties } & Kernel density $\left(\mathrm{g} / \mathrm{cm}^{3}\right)$ & 11.0 & \\
& Kernel theoretical density $\left(\mathrm{g} / \mathrm{cm}^{3}\right)$ & 11.4 & \\
& Buffer density $\left(\mathrm{g} / \mathrm{cm}^{3}\right)$ & 1.05 & \\
\cline { 2 - 4 } & Buffer theoretical density $\left(\mathrm{g} / \mathrm{cm}^{3}\right)$ & 2.25 & \\
& IPyC density $\left(\mathrm{g} / \mathrm{cm}^{3}\right)$ & $1.90 \pm 0.02$ & $\checkmark$ \\
\cline { 2 - 4 } & OPyC density $\left(\mathrm{g} / \mathrm{cm}^{3}\right)$ & $1.90 \pm 0.02$ & $\checkmark$ \\
& IPyC/OPyC BAF & $1.05 \pm 0.005$ & $\checkmark$ \\
\hline
\end{tabular}

compared. Increasing the order could improve the accuracy, but the results do not show significant change from $N_{q}=5$ to 7. To maintain a good balance between computational cost and accuracy, $N_{q}=5$ will be used for our later studies in this report. It is worth mentioning that the 1-D direct integration mode can be at least a hundred 
times faster than the MC scheme.

TABLE V. Stress correlation factors and effective mean strength for benchmark problems.

\begin{tabular}{ccccccc}
\hline \multirow{2}{*}{ Conditions } & Intact & \multicolumn{2}{c}{ IPyC cracking } & \multicolumn{3}{c}{ Asphericity } \\
\cline { 2 - 7 } & $\sigma_{\mathrm{ms}}(\mathrm{MPa})$ & $\sigma_{\mathrm{ms}}(\mathrm{MPa})$ & $\frac{\bar{\sigma}_{2-\mathrm{D}}}{\bar{\sigma}_{1-\mathrm{D}}}$ & $\sigma_{\mathrm{ms}}(\mathrm{MPa})$ & $\frac{\bar{\sigma}_{2-\mathrm{D}}}{\overline{\bar{\sigma}}_{1-\mathrm{D}}}$ & $\frac{\Delta \bar{\sigma}_{2-\mathrm{D}}}{\Delta \bar{\sigma}_{1-\mathrm{D}}}$ \\
\hline 1 & 711 & 1406 & -1.26 & 1303 & 1.54 & 1.49 \\
2 & 718 & 1405 & -1.28 & 1259 & 1.59 & 1.78 \\
3 & 717 & 1405 & -1.29 & 1405 & 1.57 & 2.58 \\
\hline
\end{tabular}

TABLE VI. Quadractic stress correlation functions for benchmark problems.

\begin{tabular}{|c|c|c|c|c|}
\hline Conditions & Type & $h_{\text {IPyC }}$ function & $\mathbf{h}_{\mathrm{SiC}}$ function & $\mathbf{h}_{\mathrm{OPyC}}$ function \\
\hline \multirow{3}{*}{1} & IPyC cracking $(\sigma)$ & $\begin{array}{c}1.0+4.89 \times 10^{3} v \\
-2.28 \times 10^{8} v^{2}\end{array}$ & $\begin{aligned} 1.0+ & 1.36 \times 10^{4} v \\
- & 6.27 \times 10^{6} v^{2}\end{aligned}$ & $\begin{array}{c}1.0-1.27 \times 10^{4} v \\
+1.84 \times 10^{8} v^{2}\end{array}$ \\
\hline & Asphericity $(\sigma)$ & $\begin{aligned} 1.0+ & 3.09 \times 10^{3} v \\
& +3.16 \times 10^{7} v^{2}\end{aligned}$ & $\begin{aligned} 1.0+ & 6.70 \times 10^{2} v \\
- & 1.19 \times 10^{8} v^{2}\end{aligned}$ & $\begin{aligned} 1.0+ & 2.89 \times 10^{3} v \\
& +4.68 \times 10^{6} v^{2}\end{aligned}$ \\
\hline & Asphericity $(\Delta \sigma)$ & $\begin{array}{c}1.0-1.52 \times 10^{3} v \\
-9.72 \times 10^{6} v^{2}\end{array}$ & $\begin{array}{r}1.0+8.69 \times 10^{3} v \\
-5.46 \times 10^{8} v^{2}\end{array}$ & $\begin{array}{c}1.0+4.58 \times 10^{2} v \\
-2.13 \times 10^{7} v^{2}\end{array}$ \\
\hline \multirow{3}{*}{2} & IPyC cracking $(\sigma)$ & $\begin{array}{c}1.0+4.26 \times 10^{3} v \\
-2.30 \times 10^{8} v^{2}\end{array}$ & $\begin{aligned} 1.0+ & 1.36 \times 10^{4} v \\
- & 1.31 \times 10^{7} v^{2}\end{aligned}$ & $\begin{array}{c}1.0-1.27 \times 10^{4} v \\
+1.87 \times 10^{8} v^{2}\end{array}$ \\
\hline & Asphericity $(\sigma)$ & $\begin{aligned} 1.0+ & 3.86 \times 10^{3} v \\
+ & 3.05 \times 10^{7} v^{2}\end{aligned}$ & $\begin{array}{r}1.0-7.92 \times 10^{2} v \\
-7.23 \times 10^{7} v^{2}\end{array}$ & $\begin{aligned} 1.0+ & 3.47 \times 10^{3} v \\
& +7.65 \times 10^{6} v^{2}\end{aligned}$ \\
\hline & Asphericity $(\Delta \sigma)$ & $\begin{aligned} 1.0+ & 2.03 \times 10^{2} v \\
+ & 1.42 \times 10^{7} v^{2}\end{aligned}$ & $\begin{array}{r}1.0+1.28 \times 10^{3} v \\
-2.49 \times 10^{8} v^{2}\end{array}$ & $\begin{aligned} 1.0+ & 2.79 \times 10^{3} v \\
+ & 6.24 \times 10^{6} v^{2}\end{aligned}$ \\
\hline \multirow{3}{*}{3} & IPyC cracking $(\sigma)$ & $\begin{array}{l}1.0+3.96 \times 10^{3} v \\
-2.35 \times 10^{8} v^{2}\end{array}$ & $\begin{array}{l}1.0+1.36 \times 10^{4} v \\
-2.30 \times 10^{6} v^{2}\end{array}$ & $\begin{array}{l}1.0-1.25 \times 10^{4} v \\
+1.82 \times 10^{8} v^{2}\end{array}$ \\
\hline & Asphericity $(\sigma)$ & $\begin{aligned} 1.0+ & 4.17 \times 10^{3} v \\
& +3.71 \times 10^{7} v^{2}\end{aligned}$ & $\begin{array}{l}1.0-6.10 \times 10^{2} v \\
-6.79 \times 10^{7} v^{2}\end{array}$ & $\begin{aligned} 1.0+ & 3.46 \times 10^{3} v \\
& +1.83 \times 10^{6} v^{2}\end{aligned}$ \\
\hline & Asphericity $(\Delta \sigma)$ & $\begin{aligned} 1.0- & 1.89 \times 10^{3} v \\
+ & 2.31 \times 10^{7} v^{2}\end{aligned}$ & $\begin{array}{r}1.0-6.38 \times 10^{3} v \\
+9.66 \times 10^{7} v^{2}\end{array}$ & $\begin{aligned} 1.0+ & 2.66 \times 10^{3} v \\
+ & 2.29 \times 10^{7} v^{2}\end{aligned}$ \\
\hline
\end{tabular}

TABLE VII. IPyC cracking probability for benchmark problems.

\begin{tabular}{cccccc}
\hline \multirow{2}{*}{ Conditions } & \multirow{2}{*}{ MC } & \multicolumn{4}{c}{ Direct Integration } \\
\cline { 3 - 6 } & & $\mathbf{N}_{q}=\mathbf{1}$ & $\mathbf{N}_{q}=\mathbf{3}$ & $\mathbf{N}_{q}=\mathbf{5}$ & $\mathbf{N}_{q}=\mathbf{7}$ \\
\hline 1 & $9.539 \times 10^{-1}$ & $9.627 \times 10^{-1}$ & $9.493 \times 10^{-1}$ & $9.492 \times 10^{-1}$ & $9.491 \times 10^{-1}$ \\
2 & $7.848 \times 10^{-2}$ & $7.533 \times 10^{-2}$ & $7.660 \times 10^{-2}$ & $7.609 \times 10^{-2}$ & $7.596 \times 10^{-2}$ \\
3 & $1.562 \times 10^{-3}$ & $1.487 \times 10^{-3}$ & $1.550 \times 10^{-3}$ & $1.545 \times 10^{-3}$ & $1.553 \times 10^{-3}$ \\
\hline
\end{tabular}

Two direct integration modes are used to compute the probability of IPyC layer cracking and SiC layer failure. The results reported in Table VIII show reasonable agreement in IPyC cracking failure probability and SiC failure probability due to IPyC cracking. The 2-D mode predicts a higher PVF probability, particularly at higher irradiation temperatures. This indicates that the correlation function for aspherical particles is less accurate than the one for particles with cracked IPyC. It is worthwhile to mention that the $\mathrm{SiC}$ failure probability of condition 3 is so low 
TABLE VIII. Failure probability for benchmark problems using Bison's direct integration scheme.

\begin{tabular}{ccccc}
\hline \multirow{2}{*}{ Conditions } & \multicolumn{4}{c}{ Simulation results with $\mathbf{N}_{q}=\mathbf{5}$} \\
\cline { 2 - 5 } & & IPyC cracking & PVF & Overall SiC \\
\hline 1 & 1-D & $9.492 \times 10^{-1}$ & 0 & $2.653 \times 10^{-3}$ \\
& 2-D & $9.932 \times 10^{-1}$ & $5.405 \times 10^{-10}$ & $2.783 \times 10^{-3}$ \\
2 & 1-D & $7.609 \times 10^{-2}$ & 0 & $2.374 \times 10^{-5}$ \\
& 2-D & $1.999 \times 10^{-1}$ & $1.295 \times 10^{-8}$ & $6.346 \times 10^{-5}$ \\
3 & 1-D & $1.545 \times 10^{-3}$ & $4.056 \times 10^{-8}$ & $5.642 \times 10^{-7}$ \\
& 2-D & $7.574 \times 10^{-3}$ & $3.194 \times 10^{-6}$ & $3.407 \times 10^{-6}$ \\
\hline
\end{tabular}

that the number of samples needed for MC simulation is beyond Bison's current practical limit of about 100 million samples. This limitation has been overcome by the direct integration mode, for which the number of samples is greatly reduced.

\section{F. AGR-2 Irradiation As-run Prediction}

The AGR-2 experiment was the second irradiation experiment performed under the Department of Energy's AGR program. The objective of AGR-2 was to irradiate both UCO and $\mathrm{UO}_{2}$ TRISO fuel to obtain normal-operation and accident-condition fuel performance data [21,22]. The AGR-2 experiment was irradiated for 559.2 effective fullpower days. Bison models use volume-averaged compact daily temperatures as boundary conditions at the outer edge of the OPyC layer. Use of daily temperatures can largely reflect the thermal state of the compacts throughout irradiation, thereby accurately predicting fission product diffusion and release. The TRISO UCO and $\mathrm{UO}_{2}$ particle geometries and material properties are listed in Table IX. The irradiation conditions of all AGR-2 compacts are listed in Table X.

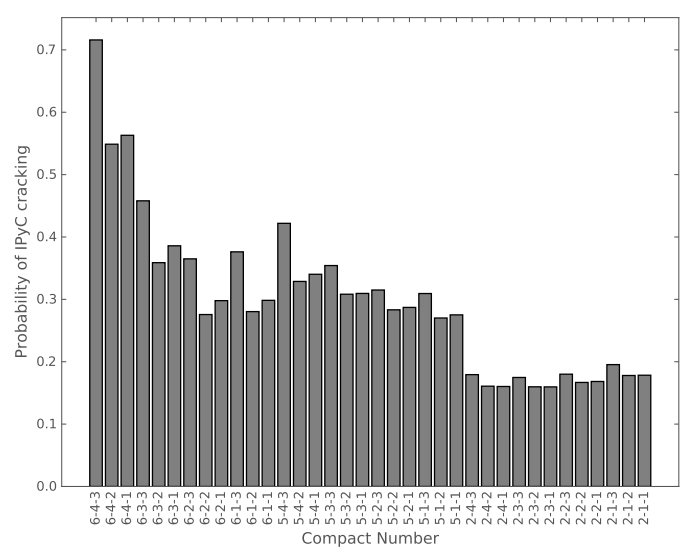

(a)

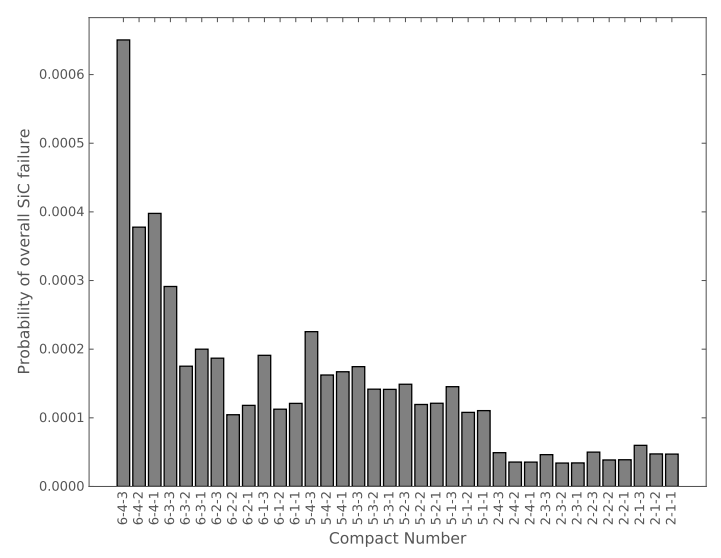

(b)

Fig. 39. UCO failure probability: (a) probability of IPyC cracking, and (b) probability of overall SiC failure.

Failure probability calculations are performed here using the 2-D direct integration mode. In this mode, the distribution of the aspect ratio can be also considered. The sampled aspect ratio is used to generate the mesh via 
TABLE IX. UCO and $\mathrm{UO}_{2}$ parameters used in modeling the AGR-2 irradiation.

\begin{tabular}{llcc}
\hline \multirow{2}{*}{ Category } & \multirow{2}{*}{ Parameter } & \multicolumn{2}{c}{ Nominal Values \pm Standard Deviation } \\
\cline { 2 - 4 } Fuel characteristics & UCO & $\mathrm{UO}_{2}$ \\
\hline \multirow{5}{*}{ Particle geometry } & ${ }^{235} \mathrm{U}$ enrichment $(\mathrm{wt} \%)$ & 14.029 & 9.600 \\
& Carbon/uranium (atomic ratio) & 0.392 & - \\
& Oxygen/uranium $($ atomic ratio) & 1.428 & 2.003 \\
\hline & Kernel diameter $(\mu \mathrm{m})$ & $426.7 \pm 8.8$ & $507.7 \pm 11.9$ \\
\cline { 2 - 4 } & Buffer thickness $(\mu \mathrm{m})$ & $98.9 \pm 8.4$ & $97.7 \pm 9.9$ \\
& IPyC thickness $(\mu \mathrm{m})$ & $40.4 \pm 2.5$ & $41.9 \pm 3.2$ \\
\cline { 2 - 4 } & SiC thickness $(\mu \mathrm{m})$ & $35.2 \pm 1.2$ & $41.9 \pm 3.2$ \\
& OPyC thickness $(\mu \mathrm{m})$ & $43.4 \pm 2.9$ & $37.5 \pm 1.2$ \\
\cline { 2 - 4 } Fuel properties & Particle aspect ratio $(\mathrm{SiC})$ & $1.037 \pm 0.011$ & $1.034 \pm 0.010$ \\
\hline & Kernel density $\left(\mathrm{g} / \mathrm{cm}^{3}\right)$ & $10.966 \pm 0.033$ & $10.858 \pm 0.082$ \\
& Kernel theoretical density $\left(\mathrm{g} / \mathrm{cm}^{3}\right)$ & 11.37 & 10.960 \\
& Buffer density $\left(\mathrm{g} / \mathrm{cm}^{3}\right)$ & 1.05 & 0.99 \\
\cline { 2 - 4 } & Buffer theoretical density $\left(\mathrm{g} / \mathrm{cm}^{3}\right)$ & 2.25 & 2.25 \\
\cline { 2 - 4 } & IPyC density $\left(\mathrm{g} / \mathrm{cm}^{3}\right)$ & $1.89 \pm 0.0011$ & $1.900 \pm 0.05$ \\
& OPyC density $\left(\mathrm{g} / \mathrm{cm}^{3}\right)$ & $1.907 \pm 0.007$ & $1.884 \pm 0.004$ \\
\cline { 2 - 4 } & IPyC BAF & $1.0465 \pm 0.0049$ & $1.0471 \pm 0.0036$ \\
& OPyC BAF & $1.0429 \pm 0.0019$ & $1.0365 \pm 0.0016$ \\
\hline
\end{tabular}

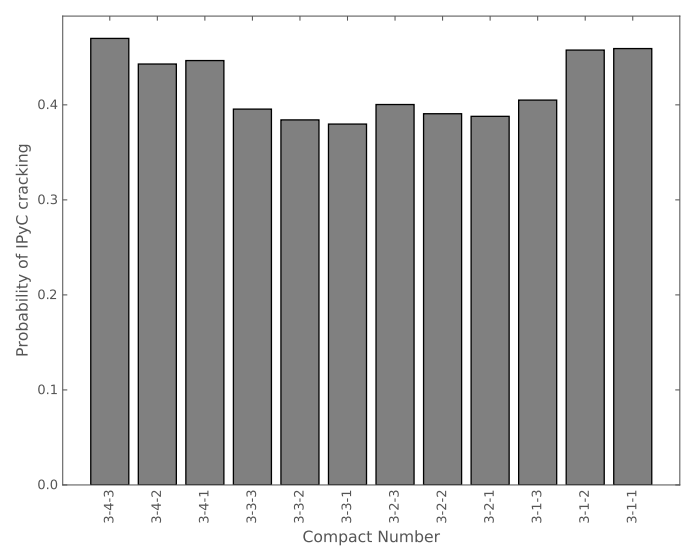

(a)

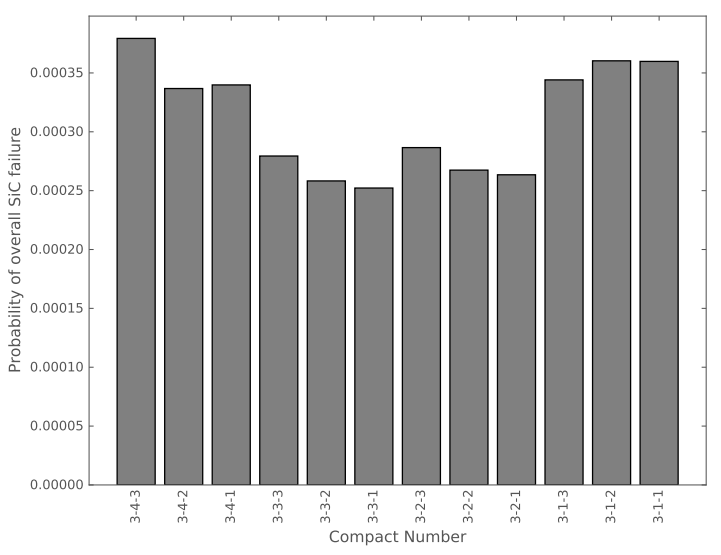

(b)

Fig. 40. $\mathrm{UO}_{2}$ failure probability: (a) probability of IPyC cracking, and (b) probability of overall SiC failure.

Bison's built-in TRISO mesh generator. Table XI summarizes the failure probabilities of IPyC cracking, PVF, and overall $\mathrm{SiC}$ failure. The PVF probability is negligible compared to that of SiC failure due to IPyC cracking. The $\mathrm{UCO}$ and $\mathrm{UO}_{2}$ failure probabilities are shown in Figure 39 and Figure 40, respectively. A linear regression is performed on UCO compacts to establish the relationship between the failure probability and irradiation conditions (i.e., temperature, fluence, and burnup). As shown in Figure 41 and Figure 42, the probability of IPyC cracking and 
TABLE X. Compacts used in modeling the AGR-2 irradiation.

\begin{tabular}{|c|c|c|c|c|c|c|c|c|c|}
\hline Compact & $\begin{array}{l}\text { Fuel } \\
\text { Type }\end{array}$ & $\begin{array}{c}\text { Fluence } \\
\left(\mathbf{1 0}^{25} \mathbf{n} / \mathbf{m}^{2}\right) \\
{[\mathbf{E}>\mathbf{0 . 1 8} \mathrm{MeV}]}\end{array}$ & $\begin{array}{l}\text { Burnup } \\
\text { (\%FIMA) }\end{array}$ & $\begin{array}{l}\text { Avg. } \\
\text { Temp. } \\
\left({ }^{\circ} \mathrm{C}\right)\end{array}$ & Compact & $\begin{array}{l}\text { Fuel } \\
\text { Type }\end{array}$ & $\begin{array}{c}\text { Fluence } \\
\left(\mathbf{1 0}^{25} \mathrm{n} / \mathbf{m}^{2}\right) \\
{[\mathbf{E}>\mathbf{0 . 1 8} \mathrm{MeV}]}\end{array}$ & $\begin{array}{c}\text { Burnup } \\
\text { (\%FIMA) }\end{array}$ & $\begin{array}{l}\text { Avg. } \\
\text { Temp. } \\
\left({ }^{\circ} \mathrm{C}\right)\end{array}$ \\
\hline $6-4-3$ & UCO & 1.94 & 7.27 & 987 & $3-4-3$ & $\mathrm{UO}_{2}$ & 3.10 & 9.33 & 998 \\
\hline $6-4-2$ & UCO & 2.21 & 9.27 & 1018 & $3-4-2$ & $\mathrm{UO}_{2}$ & 3.50 & 10.71 & 1013 \\
\hline $6-4-1$ & $\mathrm{UCO}$ & 2.20 & 9.25 & 1018 & $3-4-1$ & $\mathrm{UO}_{2}$ & 3.47 & 10.65 & 1013 \\
\hline $6-3-3$ & $\mathrm{UCO}$ & 2.14 & 7.47 & 1060 & $3-3-3$ & $\mathrm{UO}_{2}$ & 3.11 & 9.09 & 1046 \\
\hline $6-3-2$ & UCO & 2.43 & 9.61 & 1094 & $3-3-2$ & $\mathrm{UO}_{2}$ & 3.53 & 10.56 & 1062 \\
\hline $6-3-1$ & $\mathrm{UCO}$ & 2.42 & 9.60 & 1094 & $3-3-1$ & $\mathrm{UO}_{2}$ & 3.49 & 10.49 & 1062 \\
\hline $6-2-3$ & $\mathrm{UCO}$ & 2.30 & 8.23 & 1095 & $3-2-3$ & $\mathrm{UO}_{2}$ & 3.09 & 9.03 & 1045 \\
\hline $6-2-2$ & UCO & 2.61 & 10.20 & 1129 & $3-2-2$ & $\mathrm{UO}_{2}$ & 3.51 & 10.54 & 1062 \\
\hline $6-2-1$ & $\mathrm{UCO}$ & 2.60 & 10.18 & 1129 & $3-2-1$ & $\mathrm{UO}_{2}$ & 3.47 & 10.45 & 1061 \\
\hline $6-1-3$ & $\mathrm{UCO}$ & 2.42 & 9.10 & 1069 & $3-1-3$ & $\mathrm{UO}_{2}$ & 3.05 & 9.27 & 996 \\
\hline $6-1-2$ & UCO & 2.73 & 10.83 & 1100 & $3-1-2$ & $\mathrm{UO}_{2}$ & 3.45 & 10.69 & 1012 \\
\hline $6-1-1$ & UCO & 2.73 & 10.79 & 1100 & $3-1-1$ & $\mathrm{UO}_{2}$ & 3.41 & 10.62 & 1011 \\
\hline $5-4-3$ & UCO & 2.78 & 10.09 & 1040 & $2-4-3$ & UCO & 3.08 & 11.53 & 1216 \\
\hline $5-4-2$ & UCO & 3.14 & 12.05 & 1071 & $2-4-2$ & UCO & 3.47 & 13.17 & 1240 \\
\hline $5-4-1$ & UCO & 3.13 & 12.07 & 1071 & $2-4-1$ & UCO & 3.44 & 13.14 & 1240 \\
\hline $5-3-3$ & UCO & 2.91 & 10.08 & 1093 & $2-3-3$ & UCO & 3.06 & 11.02 & 1270 \\
\hline $5-3-2$ & $\mathrm{UCO}$ & 3.29 & 12.10 & 1126 & $2-3-2$ & UCO & 3.46 & 12.07 & 1296 \\
\hline $5-3-1$ & UCO & 3.28 & 12.05 & 1126 & $2-3-1$ & UCO & 3.42 & 12.65 & 1296 \\
\hline $5-2-3$ & UCO & 3.00 & 10.44 & 1108 & $2-2-3$ & UCO & 2.99 & 10.82 & 1261 \\
\hline $5-2-2$ & $\mathrm{UCO}$ & 3.39 & 12.36 & 1141 & $2-2-2$ & UCO & 3.39 & 12.57 & 1287 \\
\hline $5-2-1$ & $\mathrm{UCO}$ & 3.38 & 12.30 & 1141 & $2-2-1$ & UCO & 3.35 & 12.49 & 1287 \\
\hline $5-1-3$ & UCO & 3.03 & 11.10 & 1078 & $2-1-3$ & UCO & 2.88 & 10.96 & 1194 \\
\hline $5-1-2$ & UCO & 3.43 & 12.90 & 1109 & $2-1-2$ & UCO & 3.25 & 12.64 & 1219 \\
\hline $5-1-1$ & UCO & 3.41 & 12.82 & 1108 & $2-1-1$ & UCO & 3.21 & 12.55 & 1218 \\
\hline
\end{tabular}

overall $\mathrm{SiC}$ failure increases as temperature, fluence, and burnup decrease. The lower temperature causes less creep of the IPyC layer to offset the shrinkage and relieve tensile stress, thus increasing the probability of IPyC cracking, as well as $\mathrm{SiC}$ failure due to IPyC cracking. For all compacts, histories of the inner-wall tangential stresses in the $\mathrm{SiC}$ of an intact particle, a particle with IPyC cracking, and an aspherical particle are plotted in Figure 43 through Figure 45 . Spikes on those curves are mainly caused by fluctuations in daily temperature history. 
TABLE XI. Failure probability for AGR-2 compacts.

\begin{tabular}{|c|c|c|c|c|c|c|c|}
\hline Compact & IPyC cracking & PVF & Overall SiC & Compact & IPyC cracking & PVF & Overall $\mathrm{SiC}$ \\
\hline $6-4-3$ & $7.1592 \times 10^{-1}$ & $1.1509 \times 10^{-8}$ & $6.5044 \times 10^{-4}$ & $3-4-3$ & $4.6992 \times 10^{-1}$ & $1.1448 \times 10^{-8}$ & $3.7933 \times 10^{-4}$ \\
\hline $6-4-2$ & $5.4877 \times 10^{-1}$ & $1.3124 \times 10^{-8}$ & $3.7774 \times 10^{-4}$ & $3-4-2$ & $4.4298 \times 10^{-1}$ & $1.1135 \times 10^{-8}$ & $3.3676 \times 10^{-4}$ \\
\hline $6-4-1$ & $5.6305 \times 10^{-1}$ & $1.3422 \times 10^{-8}$ & $3.9778 \times 10^{-4}$ & $3-4-1$ & $4.4662 \times 10^{-1}$ & $1.1187 \times 10^{-8}$ & $3.3982 \times 10^{-4}$ \\
\hline $6-3-3$ & $4.5798 \times 10^{-1}$ & $1.4561 \times 10^{-8}$ & $2.9132 \times 10^{-4}$ & $3-3-3$ & $3.9553 \times 10^{-1}$ & $1.1346 \times 10^{-8}$ & $2.7942 \times 10^{-4}$ \\
\hline $6-3-2$ & $3.5883 \times 10^{-1}$ & $1.4066 \times 10^{-8}$ & $1.7520 \times 10^{-4}$ & $3-3-2$ & $3.8417 \times 10^{-1}$ & $1.1008 \times 10^{-8}$ & $2.5826 \times 10^{-4}$ \\
\hline $6-3-1$ & $3.8588 \times 10^{-1}$ & $1.4387 \times 10^{-8}$ & $1.9998 \times 10^{-4}$ & $3-3-1$ & $3.7978 \times 10^{-1}$ & $1.0983 \times 10^{-8}$ & $2.5222 \times 10^{-4}$ \\
\hline $6-2-3$ & $3.6494 \times 10^{-1}$ & $1.4667 \times 10^{-8}$ & $1.8687 \times 10^{-4}$ & $3-2-3$ & $4.0035 \times 10^{-1}$ & $1.1202 \times 10^{-8}$ & $2.8657 \times 10^{-4}$ \\
\hline $6-2-2$ & $2.7567 \times 10^{-1}$ & $1.1343 \times 10^{-8}$ & $1.0450 \times 10^{-4}$ & $3-2-2$ & $3.9065 \times 10^{-1}$ & $1.1011 \times 10^{-8}$ & $2.6744 \times 10^{-4}$ \\
\hline $6-2-1$ & $2.9795 \times 10^{-1}$ & $1.2846 \times 10^{-8}$ & $1.1813 \times 10^{-4}$ & $3-2-1$ & $3.8792 \times 10^{-1}$ & $1.0997 \times 10^{-8}$ & $2.6348 \times 10^{-4}$ \\
\hline $6-1-3$ & $3.7619 \times 10^{-1}$ & $1.4286 \times 10^{-8}$ & $1.9105 \times 10^{-4}$ & $3-1-3$ & $4.0501 \times 10^{-1}$ & $1.2732 \times 10^{-8}$ & $3.4410 \times 10^{-4}$ \\
\hline $6-1-2$ & $2.8038 \times 10^{-1}$ & $1.2311 \times 10^{-8}$ & $1.1257 \times 10^{-4}$ & $3-1-2$ & $4.5765 \times 10^{-1}$ & $1.1119 \times 10^{-8}$ & $3.6029 \times 10^{-4}$ \\
\hline $6-1-1$ & $2.9842 \times 10^{-1}$ & $1.2440 \times 10^{-8}$ & $1.2103 \times 10^{-4}$ & $3-1-1$ & $4.5924 \times 10^{-1}$ & $1.1181 \times 10^{-8}$ & $3.5980 \times 10^{-4}$ \\
\hline $5-4-3$ & $4.2191 \times 10^{-1}$ & $1.4100 \times 10^{-8}$ & $2.2551 \times 10^{-4}$ & $2-4-3$ & $1.7933 \times 10^{-1}$ & $9.6448 \times 10^{-9}$ & $4.9222 \times 10^{-5}$ \\
\hline $5-4-2$ & $3.2875 \times 10^{-1}$ & $1.4426 \times 10^{-8}$ & $1.6237 \times 10^{-4}$ & $2-4-2$ & $1.6080 \times 10^{-1}$ & $7.6879 \times 10^{-9}$ & $3.5564 \times 10^{-5}$ \\
\hline $5-4-1$ & $3.4023 \times 10^{-1}$ & $1.3955 \times 10^{-8}$ & $1.6710 \times 10^{-4}$ & $2-4-1$ & $1.6031 \times 10^{-1}$ & $7.7381 \times 10^{-9}$ & $3.5491 \times 10^{-5}$ \\
\hline $5-3-3$ & $3.5421 \times 10^{-1}$ & $1.4292 \times 10^{-8}$ & $1.7454 \times 10^{-4}$ & $2-3-3$ & $1.7477 \times 10^{-1}$ & $9.3469 \times 10^{-9}$ & $4.6364 \times 10^{-5}$ \\
\hline $5-3-2$ & $3.0824 \times 10^{-1}$ & $1.4027 \times 10^{-8}$ & $1.4174 \times 10^{-4}$ & $2-3-2$ & $1.5981 \times 10^{-1}$ & $7.3440 \times 10^{-9}$ & $3.4118 \times 10^{-5}$ \\
\hline $5-3-1$ & $3.0951 \times 10^{-1}$ & $1.3969 \times 10^{-8}$ & $1.4145 \times 10^{-4}$ & $2-3-1$ & $1.5966 \times 10^{-1}$ & $7.4163 \times 10^{-9}$ & $3.4295 \times 10^{-5}$ \\
\hline $5-2-3$ & $3.1498 \times 10^{-1}$ & $1.3842 \times 10^{-8}$ & $1.4890 \times 10^{-4}$ & $2-2-3$ & $1.8004 \times 10^{-1}$ & $9.7045 \times 10^{-9}$ & $5.0034 \times 10^{-5}$ \\
\hline $5-2-2$ & $2.8324 \times 10^{-1}$ & $1.3447 \times 10^{-8}$ & $1.1945 \times 10^{-4}$ & $2-2-2$ & $1.6680 \times 10^{-1}$ & $8.0354 \times 10^{-9}$ & $3.8616 \times 10^{-5}$ \\
\hline $5-2-1$ & $2.8710 \times 10^{-1}$ & $1.3421 \times 10^{-8}$ & $1.2121 \times 10^{-4}$ & $2-2-1$ & $1.6834 \times 10^{-1}$ & $7.9559 \times 10^{-9}$ & $3.8916 \times 10^{-5}$ \\
\hline $5-1-3$ & $3.0936 \times 10^{-1}$ & $1.4135 \times 10^{-8}$ & $1.4535 \times 10^{-4}$ & $2-1-3$ & $1.9539 \times 10^{-1}$ & $1.0612 \times 10^{-8}$ & $5.9914 \times 10^{-5}$ \\
\hline $5-1-2$ & $2.7011 \times 10^{-1}$ & $1.2928 \times 10^{-8}$ & $1.0793 \times 10^{-4}$ & $2-1-2$ & $1.7795 \times 10^{-1}$ & $9.3062 \times 10^{-9}$ & $4.7405 \times 10^{-5}$ \\
\hline $5-1-1$ & $2.7513 \times 10^{-1}$ & $1.2922 \times 10^{-8}$ & $1.1050 \times 10^{-4}$ & $2-1-1$ & $1.7838 \times 10^{-1}$ & $9.2066 \times 10^{-9}$ & $4.7167 \times 10^{-5}$ \\
\hline
\end{tabular}




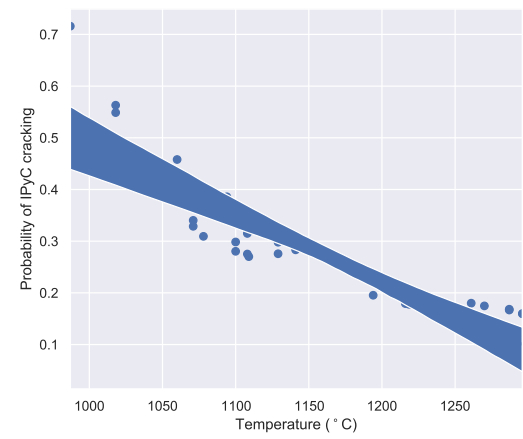

(a)

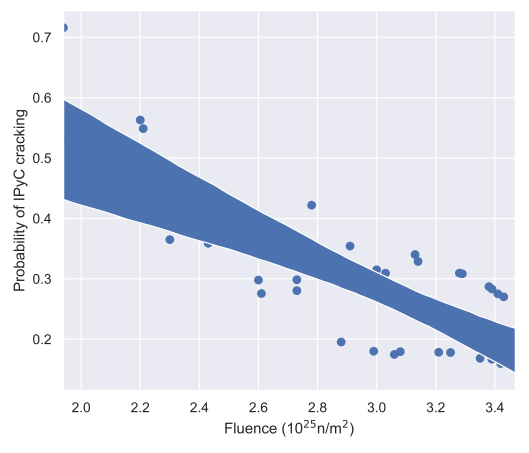

(b)

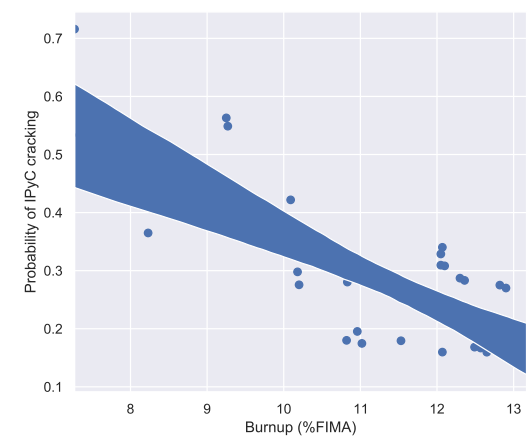

(c)

Fig. 41. Linear regression results for UCO compacts: (a) temperature vs. probability of IPyC cracking, (b) fluence vs. probability of IPyC cracking, and (c) burnup vs. probability of IPyC cracking.

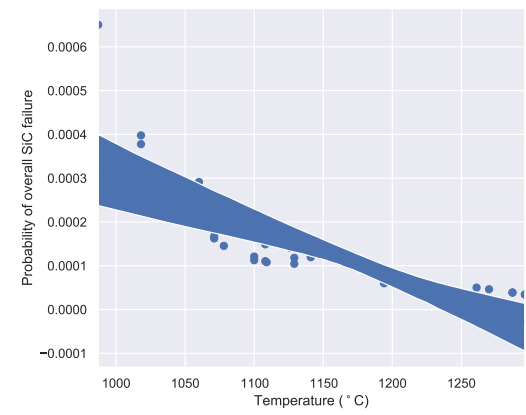

(a)



(b)

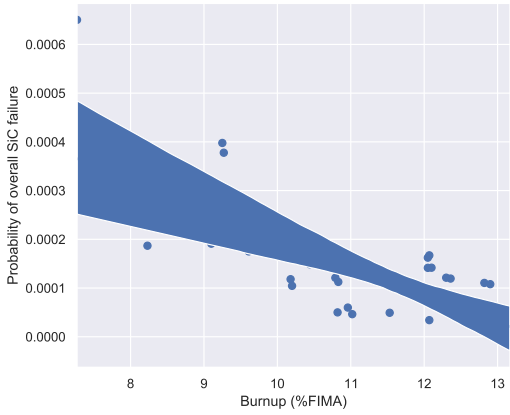

(c)

Fig. 42. Linear regression results for UCO compacts: (a) temperature vs. probability of IPyC cracking, (b) fluence vs. probability of IPyC cracking, and (c) burnup vs. probability of overall $\mathrm{SiC}$ failure. 


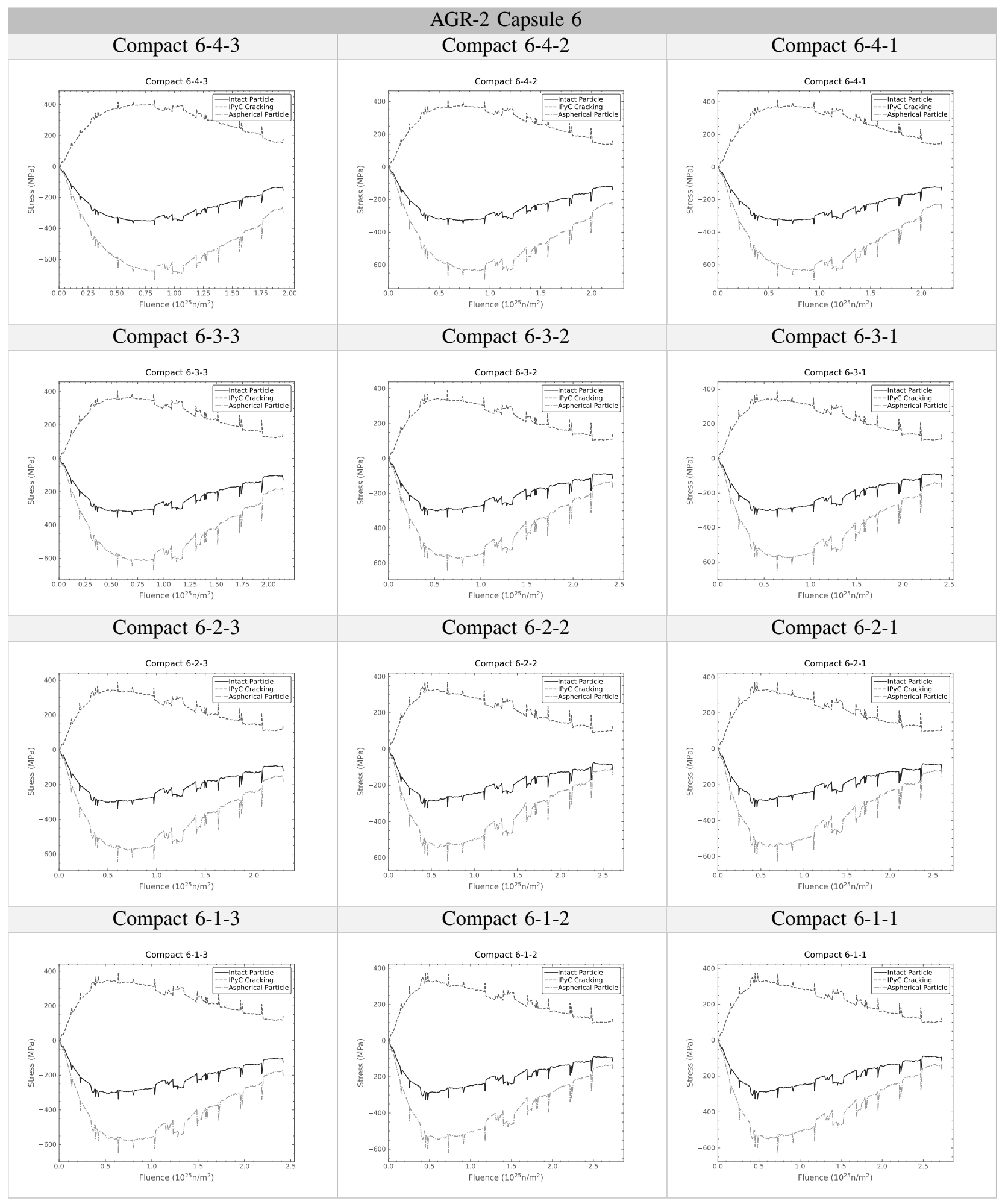

Fig. 43. Inner-wall tangential stress histories in the SiC layer for AGR-2 Capsule 6 compacts. 


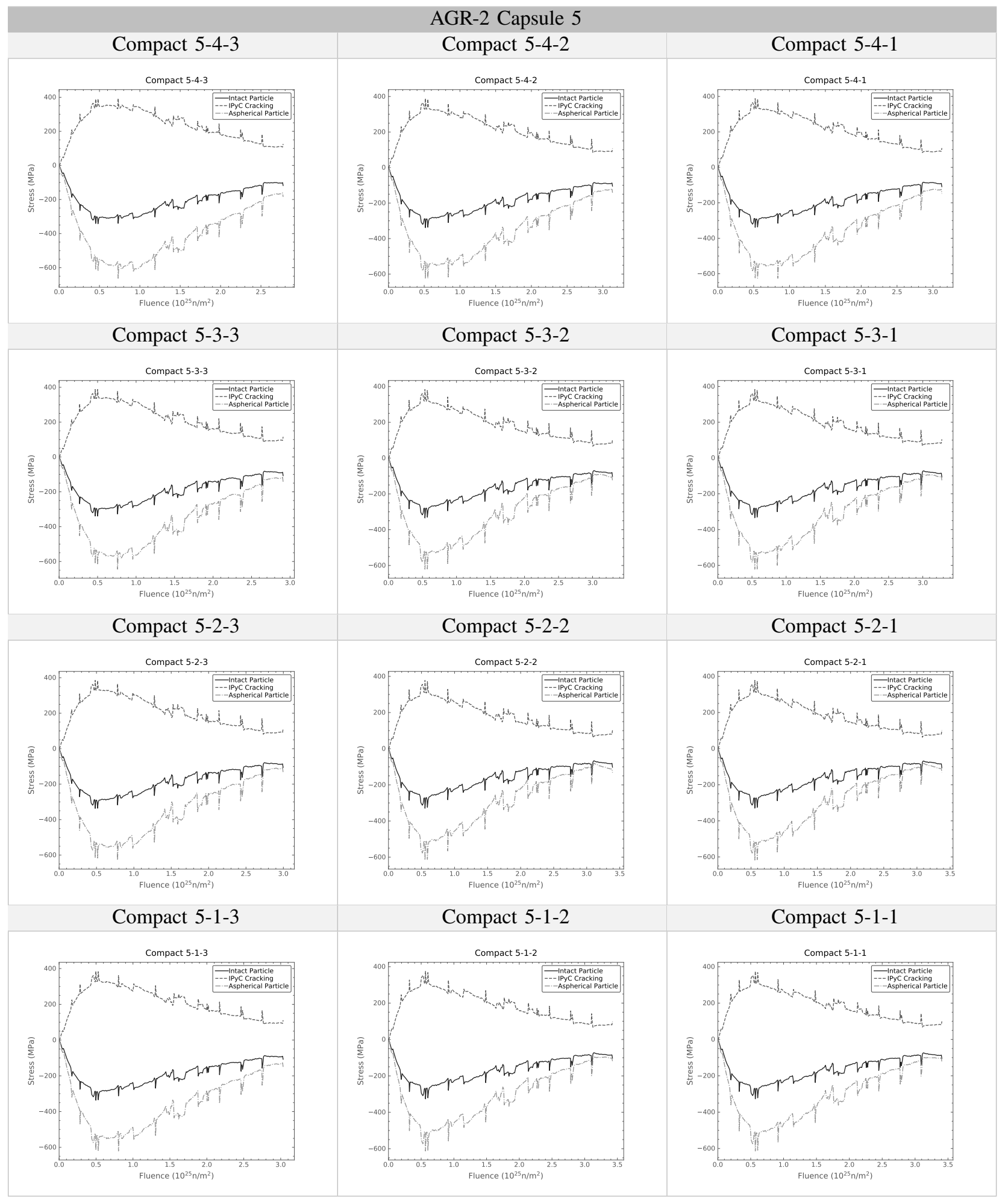

Fig. 44. Inner-wall tangential stress histories in the SiC layer for AGR-2 Capsule 5 compacts. 


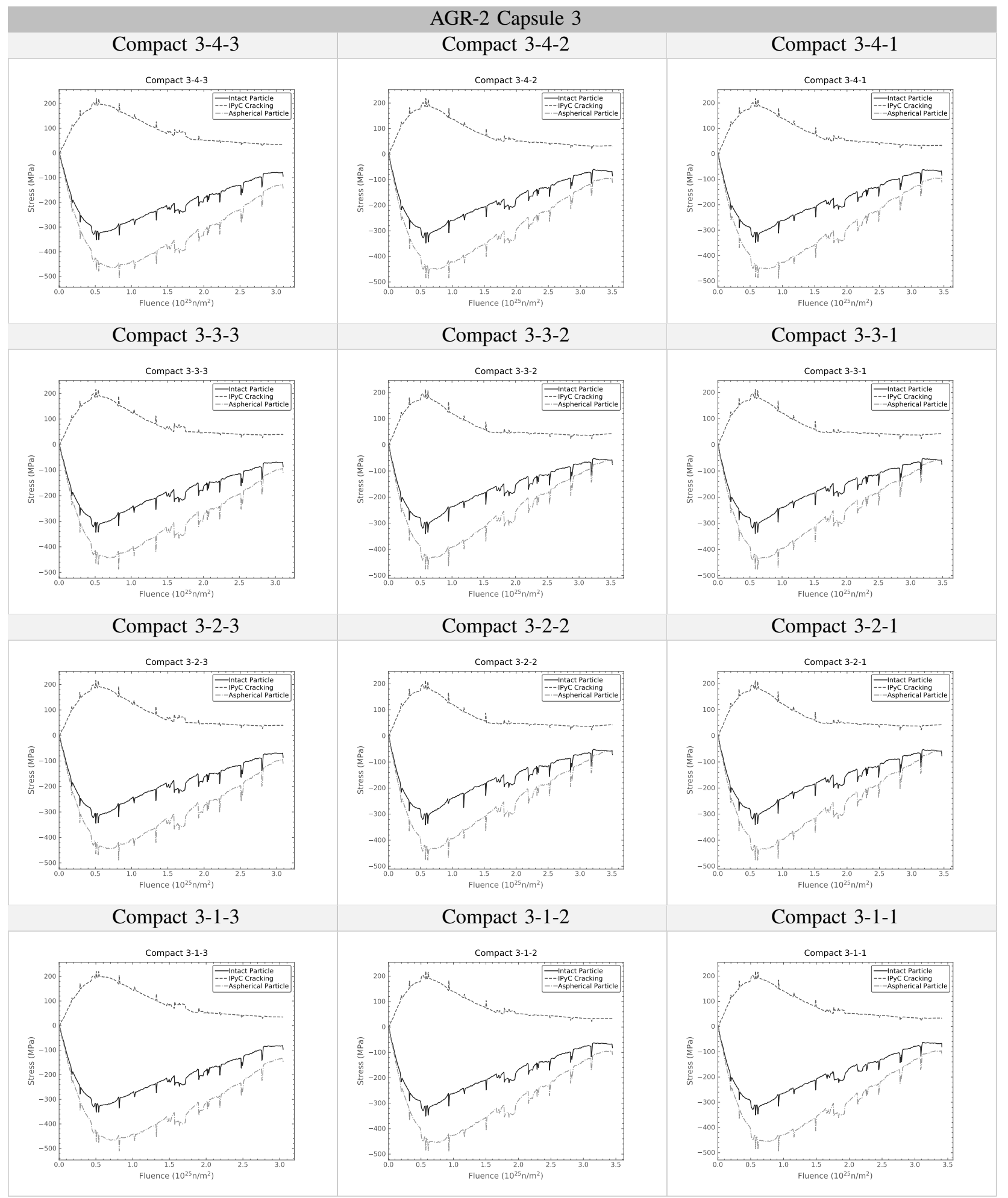

Fig. 45. Inner-wall tangential stress histories in the SiC layer for AGR-2 Capsule 3 compacts. 




Fig. 46. Inner-wall tangential stress histories in the SiC layer for AGR-2 Capsule 2 compacts. 


\section{CONCLUSION AND FUtURE WORK}

The capabilities of Bison have been significantly expanded to enable it to model the fuel performance of TRISO particles. Recent examples of such development include incorporating anisotropy in the elasticity model of PyC and using the local coordinate system for aspherical particle geometry. The capability of modeling interface debonding in TRISO particles was also developed in Bison. The effect of debonding on the thermo-mechanical behavior of TRISO particles was investigated in several numerical examples. A new capability was developed to calculate failure probability using a sparse grid sampling approach. This new approach can be over a hundred times faster than the MC scheme, particularly when the failure probability is low. It also allows for directly running high-fidelity 2-D models to yield high accuracy. All these capabilities were tested extensively, including on benchmark problems and AGR-2 as-run tests.

Future work will involve developing an anisotropic PyC thermal model in Bison. The robustness of the debonding model needs further improvement. The recently developed Mortar contact capability has yet to be introduced to model the contact between debonded layers. In addition, the debonding failure mode must be incorporated into the direct integration approach, requiring a resolution process to determine a differential particle failure probability for each of the several failure modes. 


\section{REFERENCES}

[1] R. L. Williamson et al. "BISON: A flexible code for advanced simulation of the performance of multiple nuclear forms". In: Nuclear Technology 207.7 (2021), pp. 954-980. DOI: 10.1080/00295450.2020.1836940.

[2] J.D. Hales et al. "Multidimensional multiphysics simulation of TRISO particle fuel". In: Journal of Nuclear Materials 443.1-3 (Nov. 2013), pp. 531-543. DOI: 10.1016/j.jnucmat.2013.07.070.

[3] J. D. Hales et al. BISON TRISO Modeling Advancements and Validation to AGR-1 Data. PEMP Notable Outcome 1.1.C Completion Report INL/EXT-20-59368. Idaho Falls, ID United States: Idaho National Laboratory (INL), Sept. 2020. DOI: 10.2172/1711423. URL: https://www.osti.gov/biblio/1711423/.

[4] W. Jiang et al. "TRISO particle fuel performance and failure analysis with BISON". In: Journal of Nuclear Materials 548 (May 2021), p. 152795. DOI: 10.1016/j.jnucmat.2021.152795.

[5] J. D. Hales et al. "Modeling fission product diffusion in TRISO fuel particles with BISON". In: Journal of Nuclear Materials 548 (May 2021), 152840:1-16. DOI: 10.1016/j.jnucmat.2021.152840.

[6] J. D. Hales et al. "Numerical Evaluation of AGR-2 Fission product release (under journal review)". In: (2021).

[7] A. Toptan et al. "FEA-aided investigation of the effective thermal conductivity in a medium with embedded spheres". In: Nuclear Engineering and Design 381 (Sept. 2021), p. 111355. DOI: 10.1016/j.nucengdes.2021. 111355.

[8] W. Jiang et al. Fission product transport in TRISO particles and pebbles. Tech. rep. INL/EXT-21-63549. Idaho Falls, ID United States: Idaho National Laboratory (INL), 2021. DOI: 10.2172/1818294. URL: https: //www.osti.gov/biblio/1818294.

[9] G. K. Miller et al. PARFUME Theory and Model Basis Report. Tech. rep. INL/EXT-08-14497 (Rev.1). Idaho Falls, ID (United States): Idaho National Laboratory (INL), Sept. 2018. DOI: 10.2172/1471713. URL: https://www.osti.gov/biblio/1471713-parfume-theory-model-basis-report.

[10] F. Ho. NP-MHTGR: Material Models of Pyrocarbon and Pyrolytic Silicon Carbide. Tech. rep. CEGA-002820 Rev. 1. CEGA Corporation, July 1993.

[11] P. A. Demkowicz et al. "Irradiation performance of AGR-1 high temperature reactor fuel". In: Nuclear Engineering and Design 306 (Sept. 2016). 7th International Topical Meeting on High Temperature Reactor Technology (HTR 2014), pp. 2-13. DOI: 10.1016/j.nucengdes.2015.09.011.

[12] F. J. Rice, J. D. Stempien, and P. A. Demkowicz. "Ceramography of irradiated TRISO fuel from the AGR-2 experiment". In: Nuclear Engineering and Design 329 (2018). The Best of HTR 2016: International Topical Meeting on High Temperature Reactor Technology, pp. 73-81. ISSN: 0029-5493. DOI: 10.1016/j.nucengdes. 2017.10.010.

[13] J. D. Hunn et al. "Detection and analysis of particles with failed SiC in AGR-1 fuel compacts". In: $\mathrm{Nu}$ clear Engineering and Design 306 (2016). 7th International Topical Meeting on High Temperature Reactor Technology (HTR 2014), pp. 36-46. DOI: 10.1016/j.nucengdes.2015.12.011.

[14] A. Rovinelli and M. C. Messner. Identify the influence of microstructure on mesoscale creep and fatigue damage. Tech. rep. ANL-20/49. Argonne National Laboratory, Sept. 2020. DOI: 10.2172/1658575. URL: https://www.osti.gov/biblio/1658575.

[15] P. P. Camanho and C. G. Dávila. Mixed-Mode Decohesion Finite Elements for the Simulation of Delamination in Composite Materials. Tech. rep. NASA/TM-2002-211737. National Aeronautics and Space Administration (NASA), 2002.

[16] M.L. Benzeggagh and M. Kenane. "Measurement of mixed-mode delamination fracture toughness of unidirectional glass/epoxy composites with mixed-mode bending apparatus". In: Composites Science and Technology 56.4 (1996), pp. 439-449. DOI: https://doi.org/10.1016/0266-3538(96)00005-X.

[17] A. Toptan, D. J. Kropaczek, and M. N. Avramova. "Gap conductance modeling I: Theoretical considerations on the model for single- and multi-component gases". In: Nuclear Engineering and Design 353 (Dec. 2019), 110283:1-7. DOI: 10.1016/j.nucengdes.2019.110283. 
[18] R. B. Bird, W. E. Stewart, and E. N. Lightfoot. Transport Phenomena. New York, New York: John Wiley \& Sons, Inc., 1960.

[19] T. Gerstner and M. Griebel. "Numerical integration using sparse grids". In: Numerical Algorithms 18.3 (Jan. 1998), pp. 1572-9265. DOI: 10.1023/A:1019129717644.

[20] C. J. Permann et al. "MOOSE: Enabling massively parallel multiphysics simulation". In: SoftwareX 11 (2020), p. 100430. DOI: $10.1016 /$ j.softx.2020.100430.

[21] B.P. Collin. AGR-2 Irradiation Test Plan. Tech. rep. PLN-3798 (Rev.2). Idaho National Laboratory (INL), Feb. 2018.

[22] B.P. Collin. AGR-2 Irradiation Test Final As-Run Report. Tech. rep. INL/EXT-14-32277 (Rev.4). Idaho National Laboratory (INL), Feb. 2018. DOI: 10.2172/1514716. URL: https://www.osti.gov/biblio/1514716agr-irradiation-test-final-run-report-rev. 